Aus der Klinik für Hämatologie und Medizinische Onkologie

(Prof. Dr. med. L. Trümper)

im Zentrum Innere Medizin

der Medizinischen Fakultät der Universität Göttingen

\title{
Klonale Evolution und zytogenetische Evolutionsmuster bei Myelodysplastischen Syndromen (MDS) und sekundärer akuter myeloischer Leukämie nach MDS
}

\author{
INAUGURAL-DISSERTATION \\ zur Erlangung des Doktorgrades \\ der Medizinischen Fakultät der \\ Georg-August-Universität zu Göttingen
}

vorgelegt von

Naciye Cevik

aus

Salzgitter-Bad

Göttingen 2015 
Dekan:

I. Berichterstatter/in:

II. Berichterstatter/in:

III. Berichterstatter/in:

Tag der mündlichen Prüfung: $\quad$ 23.03.2016
Prof. Dr. rer. nat. H. K. Kroemer

Prof. Dr. med. D. Haase

PD Dr. med. S. Pauli

Prof. Dr. hum. biol. M. Schön 


\section{Abkürzungsverzeichnis}

AA

ABB

A/Fall

AG

AML

ANC

CMML

ED

EPO

FAB-Klassifikation

$\mathrm{FISH}$

G-CSF

$\mathrm{Hb}$

HR

IPSS

IPSS-R

ISCN

$\mathrm{KE}$

$\mathrm{KM}$

KOM

$\mathrm{LDH}$

MDS

MDS-AL

M-W-U-Test

$\mathrm{n}$

NA

n.e.

NN

$\mathrm{p}$

$\mathrm{pB}$

q

RA ausschließlich aberrante Metaphasen

klonale zytogenetische Aberrationen

Aberrationen pro Fall

Altersgruppe

Akute myeloische Leukämie

absolute Neutrophilenzahl (absolute neutrophil count)

Chronische Myelomonozytäre Leukämie

Erstdiagnose

Erythropoietin

French-American-British-Klassifikation

Fluoreszenz-in-situ-Hybridisierung

Granulozyten-Kolonien-stimulierender Faktor

Hämoglobin

Hazard Ratio

International Prognostic Scoring System

Revised-International Prognostic Scoring System

International System for Human Cytogenetic Nomenclature

Klonale Evolution

Knochenmark

Komplex (aberrant)

Laktatdehydrogenase

Myelodysplastisches Syndrom

Akute Leukämie nach Myelodysplastischem Syndrom

Mann-Whitney-U-Test

Anzahl

normale und aberrante Metaphasen im Mosaik

nicht erreicht

ausschließlich normale Metaphasen

kurzer Arm eines Chromosoms

peripheres Blut

langer Arm eines Chromosoms

Refraktäre Anämie 
Abkürzungsverzeichnis

RAEB

RAEB-T

RARS

RCMD

t-MDS

UMG

VS.

WHO

WPSS
Refraktäre Anämie mit Exzess von Blasten

Refraktäre Anämie mit Exzess von Blasten in Transformation

Refraktäre Anämie mit Ringsideroblasten

Refraktäre Zytopenie mit multilineärer Dysplasie therapieassoziierte MDS

Universitätsmedizin Göttingen

versus

World Health Organization

WHO-adaptiertes Prognostic Scoring System 


\section{Inhaltsverzeichnis}

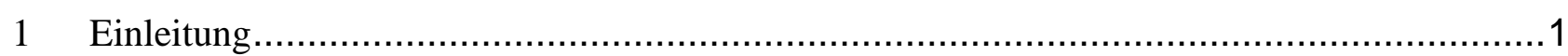

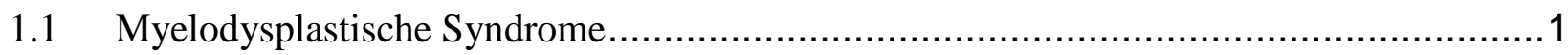

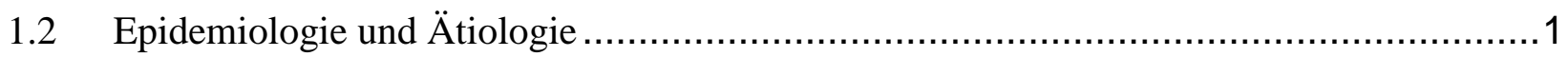

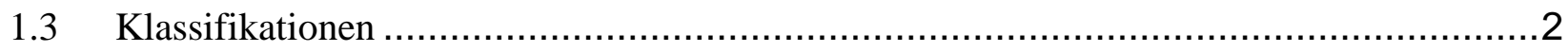

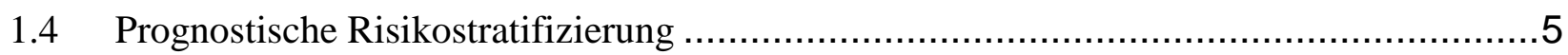

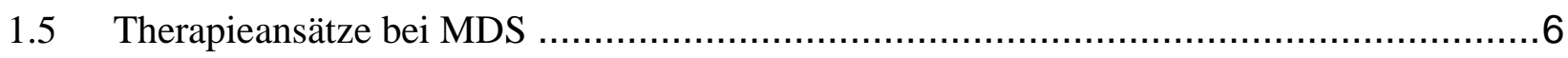

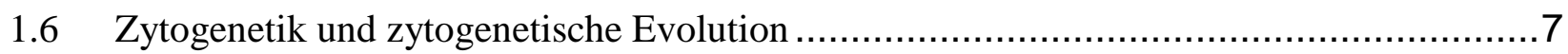

$1.7 \quad$ Pathogenese der klonalen Evolution .......................................................... 10

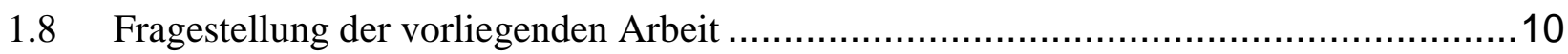

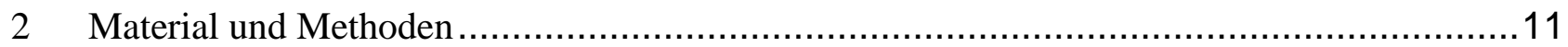

2.1 Patientenkollektiv und Datenerhebung......................................................... 11

2.2 Definition zytogenetischer Befunde .........................................................

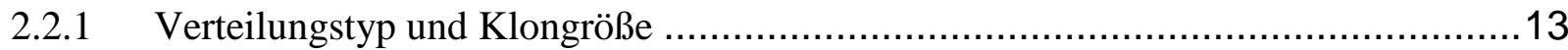

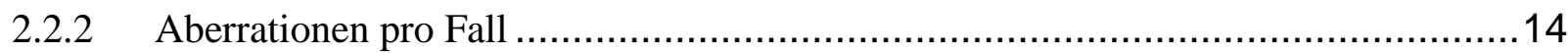

2.2.3 Theoretische klonale Evolutionsmuster ............................................... 14

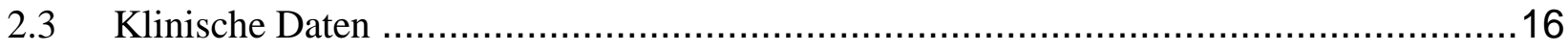

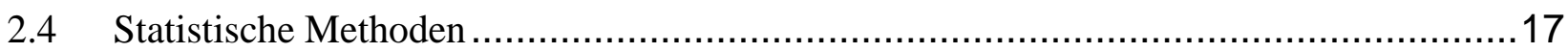

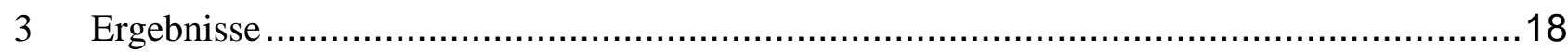

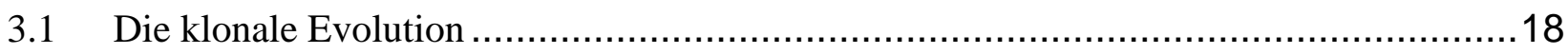

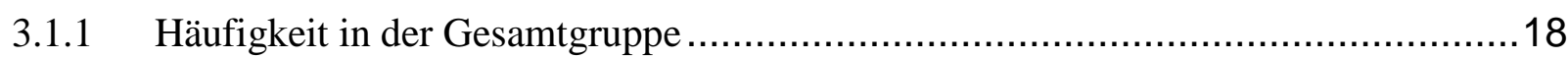

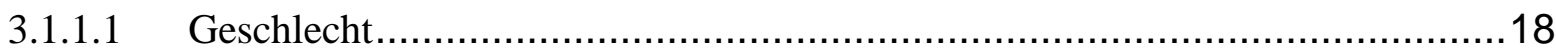

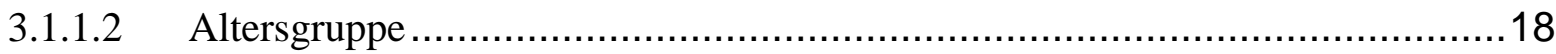

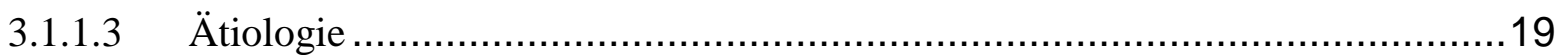

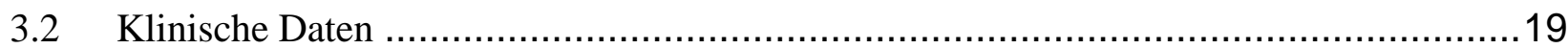

3.2.1 Gesamtgruppe zum Zeitpunkt der Erstdiagnose..........................................19

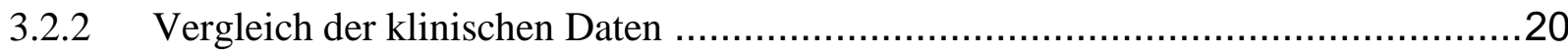

3.2.2.1 Patienten ohne klonale Evolution mit Verlaufsdaten ..............................20 
3.2.2.2 Patienten mit klonaler Evolution .22

3.2.2.3 Patienten mit klonaler Evolution im Krankheitsverlauf. .23

3.2.3 Die Prognostische Relevanz der klonalen Evolution 24

3.2.3.1 Gesamtkollektiv 24

3.2.3.2 Prognostische Relevanz der klonalen Evolution zum Diagnosezeitpunkt..... 25

3.2.4 Prognostische Relevanz des zeitlichen Auftretens der klonalen Evolution 27

3.3 Zytogenetische Befunde klonaler Evolution und ihre prognostische Bedeutung 29

3.3.1 Aberrationen pro Fall 30

3.3.2 Komplexe Aberrationen 30

3.3.3 Verteilungstyp und Klongröße 32

3.4 AML-freies Überleben innerhalb der Gruppe von Patienten mit klonaler Evolution. 35

3.4.1 Gesamtkollektiv 35

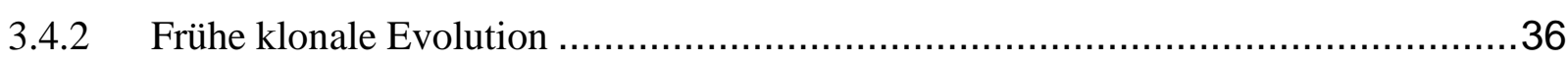

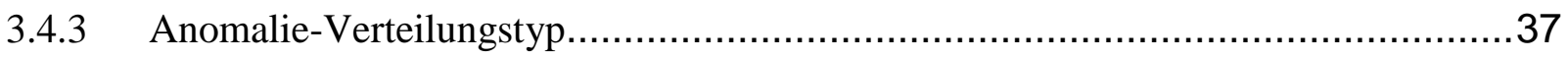

3.5 Korrelation distinkter Aberrationen mit der klonalen Evolution................................38

3.5.1 Häufigkeit spezieller zytogenetischer Aberrationen ...................................... 38

3.5.1.1 Verlust des langen Armes von Chromosom 5 (5q-) .............................. 39

3.5.1.2 Monosomie 7/Verlust des langen Armes von Chromosom 7 (7q-) .................42

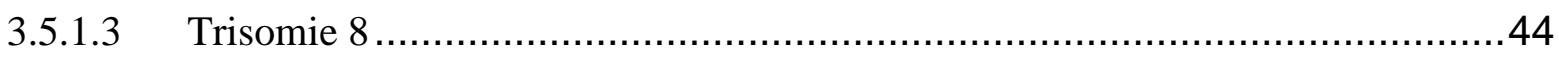

3.5.1.4 Anomalien des kurzen Armes von Chromosom 17 (17p-Anomalien) .............45

3.5.1.5 Verlust des langen Armes von Chromosom 20 (20q-) ..........................47

3.5.1.6 Verlust des Y-Chromosoms............................................................ 48

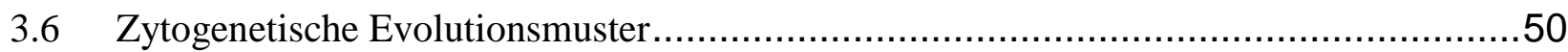

3.6.1 Häufigkeit spezifischer zytogenetischer Evolutionsmuster ............................50

3.6.1.1 Häufigkeit komplexer Anomalien innerhalb der klonalen Evolutionsmuster .....51

3.6.1.2 Auftreten distinkter Aberrationen innerhalb der klonalen Evolutionsmuster......51

3.6.1.3 Prognostische Bedeutung der klonalen Evolutionsmuster ..........................52

3.6.2 Zytogenetik klonaler Evolution im Krankheitsverlauf ..................................53 


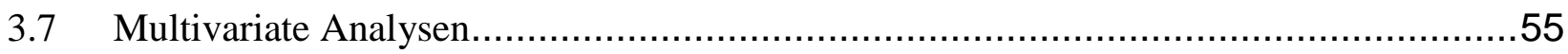

3.7.1 Gesamtüberleben in der multivariaten Analyse ....................................55

3.7.2 AML-freies Überleben in der multivariaten Analyse.................................57

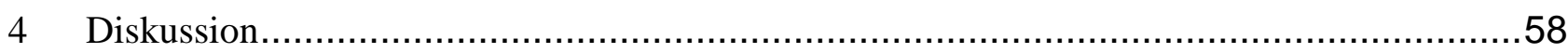

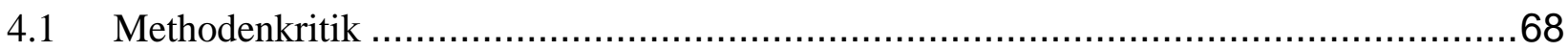

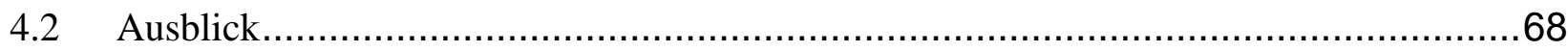

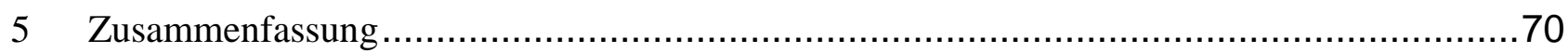

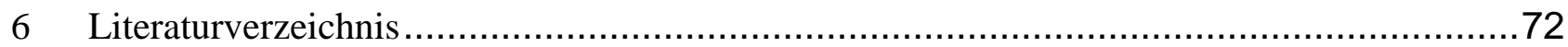

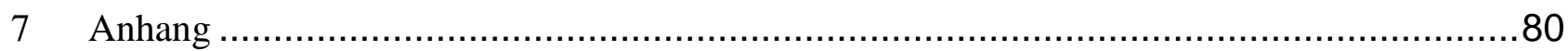

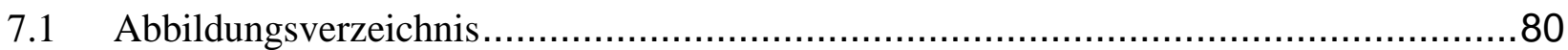

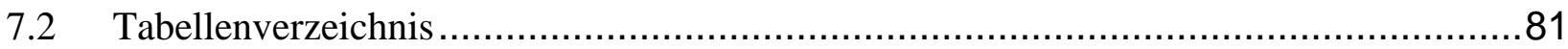




\section{$1 \quad$ Einleitung}

\subsection{Myelodysplastische Syndrome}

Myelodysplastische Syndrome (MDS) sind klonale Erkrankungen hämatopoetischer Stammzellen, welche durch eine ineffektive Hämatopoese mit Zytopenien im peripheren Blut charakterisiert sind (Janssen et al. 1989, Haase et al. 1997, Aul et al. 1998). Im Knochenmark (KM) findet sich dagegen eine Hyperzellularität mit Dysplasiezeichen der drei Zellreihen, die aus einer kompensatorisch gesteigerten Hämatopoese bei erhöhter Apoptoserate hervorgeht (Raza et al. 1997, Aul et al. 1998). Entsprechend den betroffenen Zellreihen haben die Patienten unterschiedliche Symptome wie Abgeschlagenheit, Müdigkeit, Dyspnoe, erhöhte Blutungs- oder Infektneigung (Giagounidis 2013). Der Krankheitsverlauf kann sehr unterschiedlich sein und von einer über Jahre bestehenden, nicht therapiebedürftigen Anämie bis hin zu einem raschen Übergang in eine akute myeloische Leukämie (AML) reichen (Germing und Haas 2009). In 30\% - 40\% der Fälle entsteht im Verlauf der Erkrankung eine AML (Giagounidis 2013).

\subsection{Epidemiologie und Ätiologie}

Der Altersmedian bei Erstdiagnose der Erkrankung liegt zwischen 57 und 76 Jahren (Neukirchen et al. 2009). MDS können auch bei jüngeren Patienten auftreten. Eine Studie von Kuendgen et al. (2006) konnte zeigen, dass 9\% der Patienten mit MDS jünger als 50 Jahre sind. Die Inzidenz für MDS beträgt ca. 4 auf 100.000 Einwohner pro Jahr (Neukirchen et al. 2011). Bei den über 70-jährigen Patienten liegt die Inzidenz bei 20-50 auf 100.000 Einwohner pro Jahr (Aul et al. 1992, Williamson et al. 1994, Germing et al. 2004).

Hinsichtlich ihrer Ätiologie werden MDS in primäre (idiopathische) MDS und sekundäre, meist therapieassoziierte MDS (t-MDS) unterteilt. Ursachen für das Auftreten sekundärer MDS sind frühere Chemo- und/oder Radiotherapien und Exposition gegenüber Noxen ( $z$. B. ionisierende Strahlen, Lösemittel, Haarfärbemittel) (Farrow et al. 1989, Nisse et al. 1995, Aul et al. 1998). Nicht zuletzt können auch prädisponierende genetische Faktoren das Auftreten von MDS begünstigen. Dies wird bei Patienten mit einem Fanconi-Syndrom beobachtet (Aul et al. 1998). Diese Erkrankung geht mit einer erhöhten Mutationsrate bzw. genetischen Instabilität einher. 


\subsection{Klassifikationen}

Die frühere Einteilung der MDS erfolgte zunächst nach der French-American-British (FAB)-Klassifikation, die 1982 eingeführt wurde (Bennett et al. 1982) und die MDS nach unterschiedlichen Kriterien in fünf Subtypen einteilt (Tabelle 1).

Tabelle 1: FAB-Klassifizierung der Myelodysplastischen Syndrome (Bennett et al. 1982, S. 195 - 197)

\begin{tabular}{|c|c|c|c|}
\hline FAB-Subtyp & peripheres Blut $(p B)$ & Knochenmark & weitere Kriterien \\
\hline \multirow[t]{2}{*}{$\begin{array}{l}\text { Refraktäre Anämie } \\
\text { (RA) }\end{array}$} & \multirow[t]{2}{*}{$<1 \%$ Blasten } & \multirow[t]{2}{*}{$<5 \%$ Blasten } & $\begin{array}{l}\text { pB: Retikulozytopenie, } \\
\text { Dyserythropoese }\end{array}$ \\
\hline & & & $\begin{array}{l}\text { KM: Normo- oder } \\
\text { Hyperzellularität }\end{array}$ \\
\hline $\begin{array}{l}\text { Refraktäre Anämie mit } \\
\text { Ringsideroblasten } \\
\text { (RARS) }\end{array}$ & $<1 \%$ Blasten & $<5 \%$ Blasten & $\begin{array}{l}\mathrm{KM}:>15 \% \\
\text { Ringsideroblasten }\end{array}$ \\
\hline \multirow{2}{*}{$\begin{array}{l}\text { Refraktäre Anämie mit } \\
\text { Blasten-Exzess } \\
\text { (RAEB) }\end{array}$} & \multirow[t]{2}{*}{$<5 \%$ Blasten } & \multirow[t]{2}{*}{$5-20 \%$ Blasten } & $\begin{array}{l}\text { pB: Dysplasiezeichen in } \\
\text { drei Zellreihen }\end{array}$ \\
\hline & & & KM: Hyperzellularität \\
\hline \multirow{2}{*}{$\begin{array}{l}\text { Chronische } \\
\text { myelomonozytäre } \\
\text { Leukämie } \\
\text { (CMML) }\end{array}$} & \multirow[t]{2}{*}{$<5 \%$ Blasten } & \multirow[t]{2}{*}{$<20 \%$ Blasten } & pB: >1000 Monozyten/ $\mu \mathrm{l}$ \\
\hline & & & $\begin{array}{l}\text { KM: erhöhte Anzahl an } \\
\text { Promonozyten }\end{array}$ \\
\hline $\begin{array}{l}\text { RAEB in Transformation } \\
\text { (RAEB-T) }\end{array}$ & $>5 \%$ Blasten & 21-30\% Blasten & Auerstäbchen \\
\hline
\end{tabular}

Im Verlauf wurden weitere MDS-Subgruppen definiert, die sich hinsichtlich ihrer Überlebenswahrscheinlichkeit signifikant unterscheiden. Dies führte zu einer tiefgreifenden Revision. Nachfolgend wurde eine neue Klassifikation durch die World Health Organization (WHO) im Jahre 2001 eingeführt (Bennett 2000, Jaffe et al. 2001). Insgesamt beinhaltet die WHO-Klassifikation acht Subgruppen. Unterschiede gegenüber der FAB-Klassifikation sind die Einführung der neuen Subgruppe „5q-Syndrom“ und der „unklassifizierbaren MDS“. Alle Patienten mit einem Blastenanteil von $>20-29 \%$ im Knochenmark, die vorher der Subgruppe RAEB-T zugeordnet wurden, werden in der aktualisierten WHOKlassifikation der AML zugerechnet (Greenberg et al. 1997). Die chronische myelomonozytäre Leukämie (CMML) wird hingegen in eine myeloproliferative und eine myelodysplastische Form unterteilt (Michaux und Martiat 1993, Neuwirtová et al. 1996). In der Gruppe der refraktären Anämien mit Exzess von Blasten (RAEB) werden in die Subgruppen RAEB-I (<10\% Knochenmark-Blasten) und RAEB-II $(\geq 10 \%$ KnochenmarkBlasten) unterschieden (Sanz et al. 1989, Greenberg et al. 1997). Die refraktäre Anämie 
(RA) und die refraktäre Anämie mit Ringsideroblasten (RARS) werden in rein unilineäre Dysplasien (RCUD) und multilineäre (RCMD) Dysplasien unterteilt, da sich diese hinsichtlich ihrer Prognose signifikant unterscheiden (Gattermann et al. 1990). Die aktuellste WHO-Klassifikation erschien 2008 mit einigen Neuerungen, die nachfolgend aufgelistet sind (Brunning et al. 2008).

- Der Begriff der „refraktären Anämie“ wurde durch die Bezeichnung „refraktäre Zytopenie mit unilineärer Dysplasie" ersetzt, um hier auch die Patienten mit nichterythrozytären Zytopenien und unilineärer Dysplasie erfassen zu können.

- Die Gruppen der RCMD und RCMD-RS wurden zusammengeführt und als eigene Gruppe klassifiziert.

- Die Bezeichnung „5q-Syndrom“ wurde durch die neue Bezeichnung „MDS mit del $(5 q)^{\prime \prime}$ ersetzt.

- Die Gruppe der unklassifizierbaren MDS erhielt eine neue Definition.

- Als eine eigenständige Gruppe wurde „RARS-T“ innerhalb der myelodysplastisch/ myeloproliferativen Erkrankungen definiert (Vardiman et al. 2008). 
Einleitung

Tabelle 2: WHO-Klassifikation Myelodysplastischer Syndrome (Brunning et al. 2008, S. 89)

\begin{tabular}{|c|c|c|}
\hline MDS-Subtypen & peripheres Blut & Knochenmark \\
\hline $\begin{array}{l}\text { Refraktäre Zytopenie (RCUD) } \\
\text { Unilineäre Dysplasie } \\
\text { - Refraktäre Anämie } \\
\text { - Refraktäre Neutrozytopenie } \\
\text { - Refraktäre Thrombozytopenie }\end{array}$ & $\begin{array}{l}\text { Uni- oder Bizytopenie }{ }^{1} \text {, } \\
\text { keine oder }<1 \% \\
\text { Blasten }^{2}\end{array}$ & $\begin{array}{l}<5 \% \text { Blasten, Dysplasien in } \geq 10 \% \\
\text { der Zellen einer Reihe, }<15 \% \\
\text { Ringsideroblasten }\end{array}$ \\
\hline $\begin{array}{l}\text { Refraktäre Anämie mit } \\
\text { Ringsideroblasten (RARS) }\end{array}$ & $\begin{array}{l}\text { Anämie, } \\
0 \% \text { Blasten }\end{array}$ & $\begin{array}{l}\geq 15 \% \text { Ringsideroblasten, } \\
\text { ausschließlich Dyserythropoiese, } \\
<5 \% \text { Blasten }\end{array}$ \\
\hline $\begin{array}{l}\text { Refraktäre Zytopenie mit } \\
\text { multilineären Dysplasien (RCMD) }\end{array}$ & $\begin{array}{l}\text { Zytopenie }(\mathrm{n}) \text {, } \\
\text { keine oder <1\% } \\
\text { Blasten }^{2} \\
<1000 / \mu l \text { Monozyten }\end{array}$ & $\begin{array}{l}<5 \% \text { Blasten, Dysplasien in } \\
\geq 10 \% \text { der Zellen in } 2-3 \text { Zellreihen, } \\
\pm \text { Ringsideroblasten, keine Auer- } \\
\text { stächen }\end{array}$ \\
\hline $\begin{array}{l}\text { Refraktäre Anämie mit } \\
\text { Blastenexzess-I (RAEB-I) }\end{array}$ & 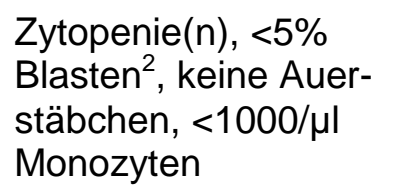 & $\begin{array}{l}\text { unilineäre oder multilineäre } \\
\text { Dysplasien, 5-9\% Blasten }{ }^{2} \text {, } \\
\text { keine Auerstäbchen }\end{array}$ \\
\hline $\begin{array}{l}\text { Refraktäre Anämie mit } \\
\text { Blastenexzess-II (RAEB-II) }\end{array}$ & 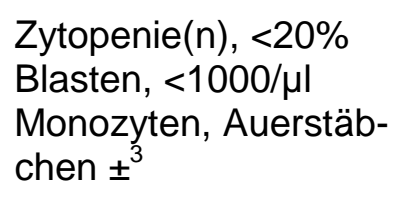 & $\begin{array}{l}\text { unilineäre oder multilineäre Dys- } \\
\text { plasien, } \\
\text { 10-19\% Blasten, Auerstäbchen } \pm^{3}\end{array}$ \\
\hline Unklassifizierte MDS (MDS-U) & $\begin{array}{l}\text { Zytopenien, } \\
\leq 1 \% \text { Blasten }^{2}\end{array}$ & $<5 \%$ Blasten \\
\hline MDS mit isolierter del(5q) & $\begin{array}{l}<1 \% \text { Blasten, Anämie, } \\
\text { Thrombozyten oft ver- } \\
\text { mehrt }\end{array}$ & $\begin{array}{l}\text { meist typische mononukleäre } \\
\text { Megakaryozyten, }<5 \% \text { Blasten, } \\
\text { isolierte del(5q) Anomalie, keine } \\
\text { Auerstäbchen }\end{array}$ \\
\hline
\end{tabular}

${ }^{1}$ Bei Vorliegen einer Panzytopenie erfolgt die Zuordnung in die Gruppe MDS-U.

${ }^{2}$ Bei $<5 \%$ KM-Blasten und 2-4\% Blasten im pB erfolgt die Einteilung in die Gruppe RAEB-I. Patienten mit 1\% Blasten im pB in den Gruppen RCUD und RCMD werden der Gruppe MDS-U zugeordnet.

${ }^{3}$ Bei Vorliegen von Auerstäbchen, $<5 \%$ Blasten im pB und $<10 \%$ Blasten im KM erfolgt die Einteilung in die Gruppe RAEB-II. 


\subsection{Prognostische Risikostratifizierung}

Zur Abschätzung der Prognose werden verschiedene Prognose-Scores eingesetzt, die unterschiedliche Parameter beinhalten. Ein sehr gut etabliertes Scoring-System stellt das International Prognostic Scoring System (IPSS) dar, welches durch die Anzahl der Zytopenien, den Blastenanteil im Knochenmark und die zytogenetischen Merkmale vier relevante prognostische Risikogruppen generiert. Diese haben sich zur Beurteilung der Prognose bewährt und sind zur Risikostratifizierung geeignet (Greenberg et al. 1997). Eine überarbeitete Version des IPSS stellt das IPSS-R (Revised International Prognostic Scoring System) dar (Greenberg et al. 2012) (Tabelle 3). Auch hier sind die Anzahl der Zytopenien, der Blastenanteil im Knochenmark und zytogenetische Merkmale die prognostisch relevanten Parameter. Neuerungen zeigen sich in den Definitionen der zytogenetischen Risikogruppen, die nun aus fünf statt der bisher drei zytogenetischen Risikogruppen bestehen (Schanz et al. 2012). Daneben wurden neue Grenzwerte für den Knochenmark-Blastenanteil und die Zytopenien festgelegt, wobei hier erstmals auch das Ausmaß der Zytopenien berücksichtigt wurde. Insgesamt werden im IPSS-R fünf klinisch relevante Risikogruppen unterschieden. Ein weiteres Scoring-System stellt das WHOadaptierte Prognostic Scoring System (WPSS) dar (Tabelle 4). In diesem werden auch die Transfusionsabhängigkeit und die WHO-Subtypen als weitere Prognoseparameter zur Zuordnung der MDS-Patienten in die verschiedenen Risikogruppen berücksichtigt (Malcovati et al. 2007, Malcovati et al. 2011).

Tabelle 3: Revision des International Prognostic Scoring System (IPSS-R) (Greenberg et al. 2012, S. 2458)

\begin{tabular}{lccccccc}
\hline Score & $\mathbf{0}$ & $\mathbf{0 , 5}$ & $\mathbf{1 , 0}$ & $\mathbf{1 , 5}$ & $\mathbf{2 , 0}$ & $\mathbf{3 , 0}$ & $\mathbf{4 , 0}$ \\
$\begin{array}{l}\text { Zytogenetische Risi- } \\
\text { kogruppe }\end{array}$ & $\begin{array}{c}\text { sehr } \\
\text { gut }\end{array}$ & - & gut & - & intermediär & ungünstig & $\begin{array}{c}\text { sehr un- } \\
\text { günstig }\end{array}$ \\
\hline $\begin{array}{l}\text { Knochenmark-Blasten } \\
(\%)\end{array}$ & $\leq 2$ & - & $>2-<5$ & - & $5-10$ & $>10$ & - \\
\hline Hämoglobin g/dl & $\geq 10$ & - & $8-<10$ & $<8$ & - & - & - \\
\hline $\begin{array}{l}\text { Thrombozyten }\left(\times 10^{9} /\right) \\
\text { ANC }\left(\times 10^{9} / \text { /) (absolute }\right.\end{array}$ & $>0,8$ & $50-<100$ & $<50$ & - & - & - & - \\
Neutrophilenzahl) & $<0,8$ & - & - & - & - & - \\
\hline
\end{tabular}

Einteilung der Patienten in fünf Risikogruppen nach den ermittelten Scoringpunkten: "very low risk": $\leq 1,5$; "low risk": >1,53; "intermediate risk": >3-4,5; "high risk": >4,5-6; "very high risk": >6. 
Tabelle 4: WHO-adaptiertes Prognostic Scoring System (WPSS) (Malcovati et al. 2011, S. 1437)

\begin{tabular}{lllll}
\hline Score & $\mathbf{0}$ & $\mathbf{1}$ & $\mathbf{2}$ & $\mathbf{3}$ \\
WHO-Typ & RCUD/RARS/ & RCMD & RAEB-I & RAEB-II \\
& $\begin{array}{l}\text { MDS mit isolierter } \\
\text { del }(5 \mathrm{q})\end{array}$ & & & \\
\hline $\begin{array}{l}\text { zytogenetische } \\
\text { Risikogruppe }\end{array}$ & gut & intermediär & ungünstig & - \\
\hline Transfusion & & & & \\
\hline
\end{tabular}

* Niedrig-Risiko: normaler Karyotyp,5q-,20q-,-Y; Hoch-Risiko: komplexer Karyotyp, Chromosom 7-Anomalien; Intermediäres Risiko: alle anderen Aberrationen

** Transfusionsbedarf: Männer $\mathrm{Hb}<9 \mathrm{~g} / \mathrm{dl}$, Frauen $\mathrm{Hb}<8 \mathrm{~g} / \mathrm{dl}$

\subsection{Therapieansätze bei MDS}

Abhängig vom Risikoprofil des Patienten, welches über die beschriebenen ScoringSysteme erfasst wird, ist eine an die Prognose adaptierte Therapie möglich und aufgrund der sehr heterogenen Krankheitsverläufe der MDS auch erforderlich.

Niedrig-Risiko-Patienten: Für die Niedrig-Risiko-Subgruppen stehen Therapiemöglichkeiten im Rahmen einer supportiven Therapie zur Verfügung. Hierbei ist die symptomatische Behandlung der Zytopenien mit Erythrozytenkonzentraten und/oder Thrombozytenkonzentraten im Vordergrund. Eine Eisenchelattherapie wird zusätzlich bei polytransfundierten eisenüberladenen Patienten angewendet, um Folgeschäden durch vermehrte Eisenablagerung zu vermeiden. Ein weiteres supportives Vorgehen, insbesondere bei ausgeprägten Zytopenien, stellt die Gabe von Wachstumsfaktoren wie EPO (Erythropoietin) oder G-CSF (Granulozyten-Kolonie-stimulierender Faktor) sowie die Gabe von Antibiotika und/oder Antimykotika zur Vorbeugung von Infektionen dar (Germing et al. 2013, Hofmann et al. 2013, Malcovati et al. 2013). Patienten mit einer isolierten 5q-minusAnomalie der Niedrig- und Intermediär-1-Risikogruppe nach IPSS mit transfusionspflichtiger Anämie können mit dem immunomodulierenden Medikament Lenalidomid behandelt werden (Germing et al. 2013). Das Ansprechen beträgt für Patienten mit del(5q) 67\% (List et al. 2006).

Hoch-Risiko-Patienten: Bei nach Alter und biologischen Faktoren geeigneten Patienten mit einem intermediären-II oder hohem Risiko nach IPSS ohne bedeutende Begleiterkrankungen kommt die allogene Stammzelltransplantation in Frage. Sie stellt bisher die einzig kurative Therapie dar (Platzbecker et al. 2012). MDS-Patienten, die nicht für eine allogene Transplantation geeignet sind, können mit demethylierenden Substanzen wie 5Azazytidine oder Decitabine behandelt werden. Bei Hoch-Risiko-Patienten mit günstigem 
Karyotyp kann eine Induktionschemotherapie eingesetzt werden (Germing et al. 2013, Hofmann et al. 2013, Malcovati et al. 2013). Hierbei handelt es sich um eine Polychemotherapie, die der Therapie der AML entspricht. Die Rate an dauerhaften Remissionen ist unter anderem abhängig vom initialen Karyotyp, Geschlecht und LDH (Laktatdehydrogenase)-Serumspiegel (Giagounidis 2013), aber meist niedrig. Zurzeit werden weitere Substanzen in klinischen Studien hinsichtlich ihrer Wirksamkeit geprüft.

\subsection{Zytogenetik und zytogenetische Evolution}

MDS weisen in etwa $50 \%$ der Fälle klonale zytogenetische Aberrationen auf (Toyama et al. 1993, Solé et al. 2005, Haase et al. 2007, Haase 2008). Die Zytogenetik ist ein wichtiger und in vielen Studien gut validierter Prognoseparameter (Greenberg et al. 1997, Germing et al. 2005, Solé et al. 2005, Haase et al. 2007, Schanz et al. 2012). In den letzten Jahren wurden die Patientenkollektive, in denen zytogenetische Aberrationen untersucht wurden, zunehmend größer. In der aktuellsten Studie wurden von Schanz et al. (2012) die Daten von über 2900 Patienten ausgewertet. MDS sind unter anderem durch die Vielzahl der zytogenetischen Aberrationen charakterisiert. Hierbei stehen unbalancierte Anomalien (häufig Deletionen und Monosomien, weniger häufig Trisomien) im Vordergrund (Haase 2008).

Die häufigste Aberration ist eine Deletion im langen Arm des Chromosoms 5 (5q-). Diese ist bei ca. 30\% der Patienten nachweisbar (Greenberg et al. 1997, Haase et al. 2007, Haase 2008). Die zweithäufigste Aberration stellt mit ca. 25\% der Fälle die Monosomie 7 dar und weitere häufige Chromosomenanomalien sind die Trisomie 8, die partielle oder totale Monosomie 20 (-20/20q-), der Verlust des Y-Chromosoms sowie die Trisomie 21 (White et al. 1994, Solé et al. 2000, Haase et al. 2007, Haase 2008). Die Chromosomenanomalien können isoliert oder innerhalb von komplexen Aberrationen ( $\geq 3$ Aberrationen) vorkommen, die für die Prognose des betroffenen Patienten eine wichtige Rolle spielt. Komplexe Chromosomenanomalien machen 15\% der Fälle aus. Nur von den aberranten Fällen ausgehend, liegt der Anteil der komplexen Anomalien bei ca. $30 \%$ und das Auftreten einer klonalen Evolution steht im engen Zusammenhang mit komplexen Anomalien (Greenberg et al. 1997, Solé et al. 2005, Haase et al. 2007, Haase 2008). Als klonale Evolution wird laut der ISCN-Nomenklatur (International System for Human Cytogenetic Nomenclature) der Nachweis von zusätzlichen Chromosomenanomalien bei bereits vorhandener primärer Aberration eines Zellklons definiert (Shaffer et al. 2013). Die klonale Evolution kann durch sequentielle Chromosomenanalysen diagnostiziert werden. Zusätz- 
lich kann die Karyotypevolution auch in Metaphasen, die die Evolutionsschritte zu ein und demselben Zeitpunkt aufzeigen, in einer einzigen zytogenetischen Untersuchung erfasst werden (Schanz 2003, Haase 2005, Haase 2008). In der folgenden Abbildung 1 wird dies schematisch dargestellt:

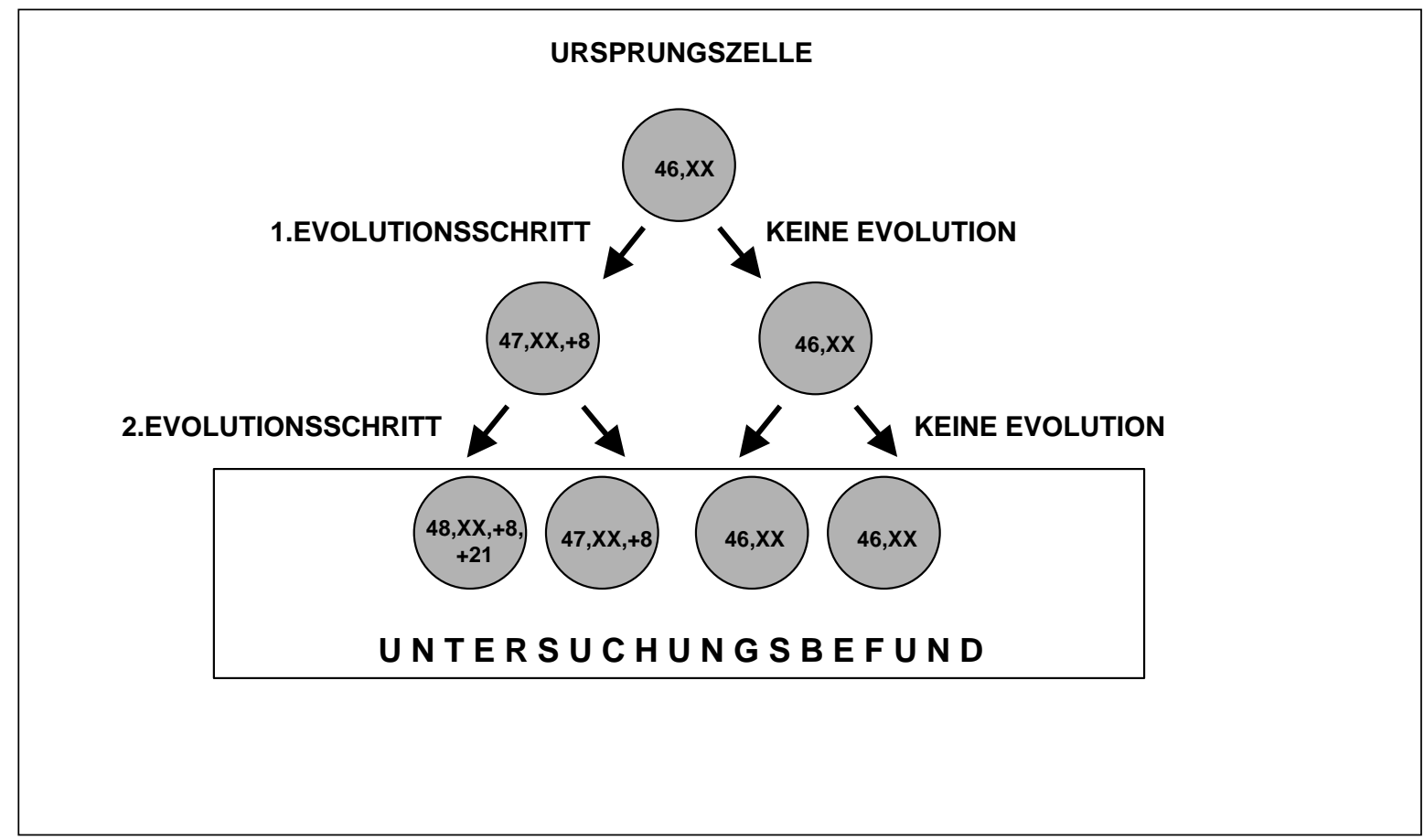

Abbildung 1: Exemplarische Darstellung einer klonalen Evolution (Schanz 2003, S. 20. Mit freundlicher Genehmigung des Autors)

Die Inzidenz für eine klonale Evolution wird in der Literatur mit $11,9 \%$ bis zu $39,0 \%$ angegeben (Horiike et al. 1988, White et al. 1994, Tien et al. 1995, Lai et al. 2004, Bernasconi et al. 2010, Wang et al. 2010). Häufig wird außer Acht gelassen, dass das Krankheitsbild der MDS hinsichtlich der Genetik eine fortschreitende Erkrankung darstellt und in den bisher publizierten Studien wird der Karyotyp oft nur zum Zeitpunkt der Erstdiagnose berücksichtigt (Haase et al. 2011). 1985 wurde von Tricot et al. eine Studie publiziert, die sich mit MDS-Evolution befasst. In dieser beschreibt der Autor verschiedene Evolutionsmuster und die damit assoziierten unterschiedlichen klinischen Verläufe der MDS. Dabei bezieht sich das Tricot-Modell im Wesentlichen auf den Blastenanteil (Abbildung 2). 


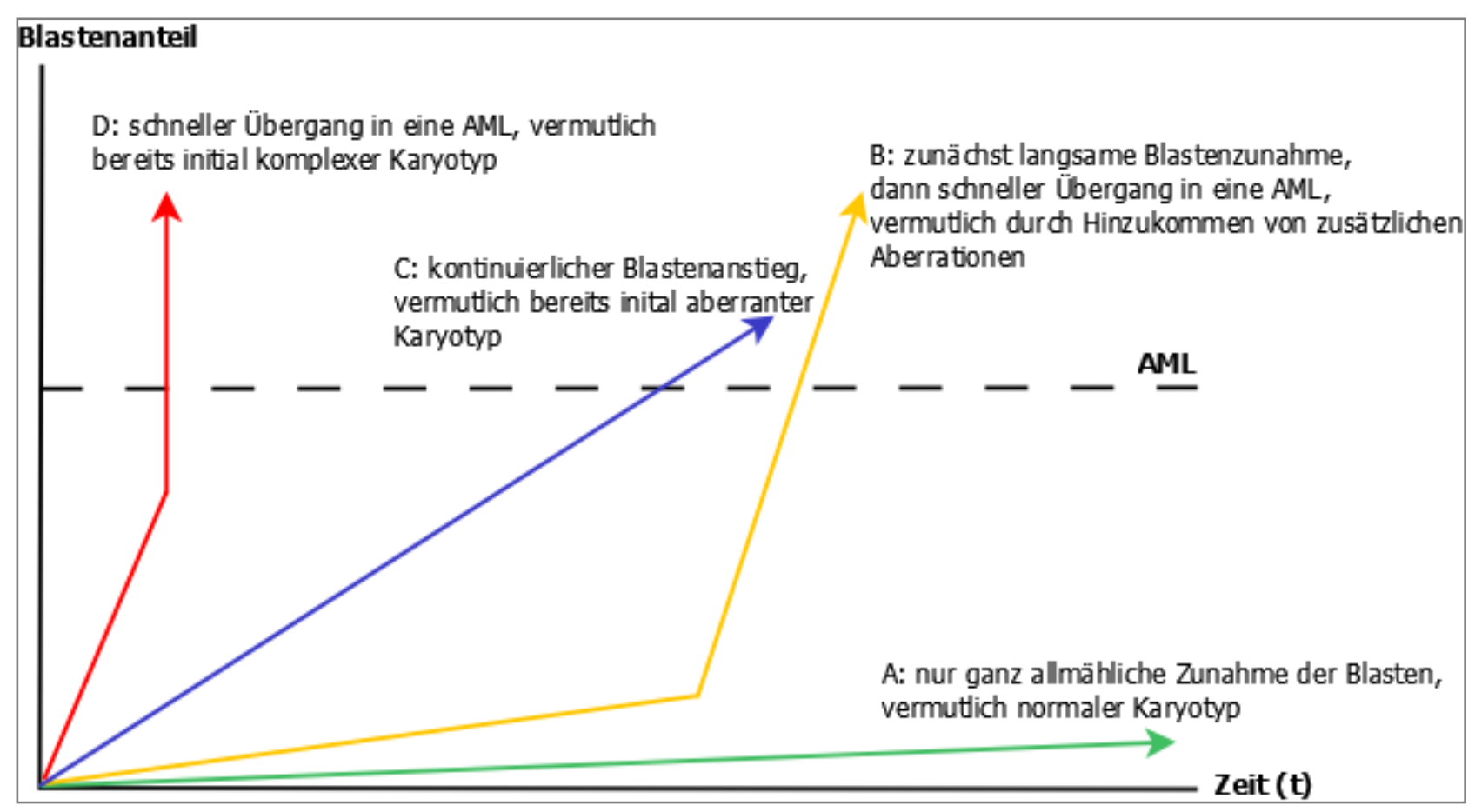

Abbildung 2: Muster der MDS-Evolution (Tricot et al. 1985, S. 668. Mit freundlicher Genehmigung des Autors)

MDS-Patienten mit stabilem Klon und geringer Proliferationskapazität (minimaler oder kein Anstieg der Blasten im Knochenmark) werden dem Typ A zugeordnet. Zum Zeitpunkt der Diagnose hat die Mehrheit dieser Patienten vermutlich einen normalen Karyotyp. Zum Typ B werden MDS-Patienten mit initial geringem Blastenanstieg gezählt, welche im Verlauf aber einen raschen Übergang in eine AML zeigen. Vermutlich weist ein Großteil dieser Patienten zum Zeitpunkt der Diagnose bereits Aberrationen auf. Mit hinzukommenden oder neuen Aberrationen führt dieses Ereignis dann wahrscheinlich zum raschen und abrupten Übergang in eine AML. Im Typ $C$ werden Patienten mit einem kontinuierlichen Blastenanstieg zusammengefasst, die letztlich eine AML, vermutlich ohne das Auftreten zusätzlicher Aberrationen, entwickeln. Der klinische Verlauf der vierten Gruppe (Typ D) zeigt ein deutlich verkürztes Progressionsintervall mit raschem Blastenansteig. Diese Patienten haben vermutlich bei Erkrankungsbeginn bereits einen komplex aberranten Karyotyp.

In den folgenden Jahren wurden einige weitere Studien zu sequentiellen zytogenetischen Untersuchungen in kleineren Patientenkollektiven ( $n=30$ bis 150) zum Thema Karyotypevolution veröffentlicht, die in allen Studien mit einem ungünstigeren Krankheitsverlauf verbunden war (Horiike et al. 1988, Geddes et al. 1990, White et al.1994, Bernasconi et al. 2010, Wang et al. 2010). Die bisher größte Studie von Haferlach et al. zu 
diesem Thema konnte zusätzlich zeigen, dass die Karyotypevolution mit einer Progression des Karyotyps und einem ungünstigeren IPSS-Risiko assoziiert ist (Haferlach et al. 2011).

\subsection{Pathogenese der klonalen Evolution}

Ein möglicher Erklärungsversuch für die Entstehung der klonalen Evolution ist das Modell der Mehrschritt-Pathogenese auf der Stammzellebene (Raskind et al. 1984, Cermák et al. 2005, Haase 2005, Haase et al. 2011). Es kommt zur Aktivierung von Onkogenen und Inaktivierung von Tumorsuppressorgenen mit Dysregulation der Zellproliferation und der Apoptose (Parker et al. 2001, Nolte und Hofmann 2009, Janka-Schaub und Haase 2009). Hieraus resultieren Chromosomeninstabilitäten, die im Verlauf der Erkrankung zu nachweisbaren klonalen Chromosomenaberrationen führen (Haase et al. 1992, Haase 2005). Das sequentielle Auftreten von Aberrationen und die Akkumulation dieser Veränderungen führen dann zu einer nachweisbaren klonalen Evolution (Haase 2005). Neben dieser Theorie der Akkumulierung von Chromosomenaberrationen, gibt es das Konzept der "Chromothripsis" (Stephens et al. 2011). Dieser Theorie liegt zugrunde, dass ein einziges Ereignis, welches als „zelluläre Krise“ beschrieben wird, zu multiplen Chromosomenumlagerungen führt. Dieses Phänomen ließ keine Rückschlüsse auf einen graduellen Prozess zu und wird bei 2-3\% aller Tumoren sowie in $25 \%$ aller Knochentumoren vermutet.

\subsection{Fragestellung der vorliegenden Arbeit}

Ziel der vorliegenden Arbeit ist es, fundierte Kenntnisse über die bei MDS auftretenden zytogenetischen Evolutionsverläufe zu erhalten. Die Auswertung sowohl der klinischen als auch zytogenetischen Daten eines Kollektivs von 729 Patienten, hiervon 225 Patienten mit Verlaufsdaten, soll zur Klärung der prognostischen Relevanz der Karyotypevolution beitragen. Es liegen nur wenige Studien vor, die MDS als eine fortschreitende Krankheit erfassen und die Patienten im Verlauf beobachten. In der hier vorliegenden Arbeit sollen folgende Fragestellungen untersucht werden:

1. Wie häufig kommen klonale Evolutionen bereits bei Erstuntersuchung in dem hier untersuchten Patientenkollektiv vor und wie häufig treten sie innerhalb von sequentiellen Untersuchungen auf?

2. Bestehen Unterschiede bezüglich der klinischen Verläufe zwischen Patienten mit klonaler Evolution und solchen ohne klonale Evolution? 
3. Gibt es Korrelationen zwischen der klonalen Evolution und allgemeinen zytogenetischen Veränderungen, wie dem Auftreten von komplexen Aberrationen, dem Verteilungstyp, der Anzahl der Aberrationen pro Fall und sind diese Korrelationen von prognostischer Bedeutung?

4. Bestehen prognostische Unterschiede innerhalb der klonalen Evolution abhängig vom zeitlichen Auftreten?

5. Bestehen definierbare klonale Evolutionsmuster und sind diese von prognostischer Relevanz?

6. Gibt es Unterschiede hinsichtlich der Häufigkeit und der prognostischen Bedeutung bestimmter Aberrationen innerhalb der klonalen Evolution?

\section{Material und Methoden}

\subsection{Patientenkollektiv und Datenerhebung}

Der vorliegenden Arbeit liegt eine retrospektive Analyse zugrunde, die sich auf insgesamt 729 Patientinnen und Patienten mit nachgewiesenem MDS oder sekundärer AML nach MDS aus dem Zeitraum 1982 bis 2009 bezieht. Im Rahmen der Diagnostik wurden bei diesen Patienten zytogenetische Analysen aus Knochenmarkproben durchgeführt. Zusätzlich wurden laborchemisch relevante Parameter erhoben. Die Aufarbeitung und die Analyse der Proben wurden unter der Leitung von Prof. Dr. med. D. Haase in den zytogenetischen Laboren des Universitätsklinikums Lübeck bis 1992 und ab 1992 an der Universitätsmedizin Göttingen durchgeführt.

Die Auswertung der Daten für die Studie erfolgte anonymisiert. Diese wurden in einer Excel-Datenbank (Microsoft Office Excel 2007) erfasst. Die Datenbank wurde von Frau PD Dr. med. J. Schanz mit Daten bis 2003 zur Verfügung gestellt. Diese wurde ergänzt und fortgeführt. Die Richtlinien des Datenschutzes sowie der Schweigepflicht wurden eingehalten. Neben den Basisdaten von Patienten (Alter, Geschlecht) wurden zytogenetische Befunde (Aberrationen pro Fall, komplexe und nicht-komplexe Aberrationen, Verteilungstyp und Klongröße) erfasst. Zudem wurden Laborparameter (Blutbild, Blastenanteil KM/pB), Überlebensdaten und Therapien dokumentiert. Anhand der zytogenetischen Primärdaten und den Verlaufsdaten wurden theoretische klonale Evolutionsmuster generiert. Es wur- 
den keine zusätzlichen Untersuchungen an den Patienten durchgeführt oder Patienten kontaktiert.

Untersucht wurden 429 männliche Patienten (58,8\%) und 300 weibliche Patienten (41,2\%) mit einem Altersmedian von 65,6 Jahren zum Zeitpunkt der Erstdiagnose. Der Großteil der in der Studie erfassten Patienten ( $n=663,90,9 \%$ ) hatte ein primäres (idiopathisches) MDS. 66 Patienten $(9,1 \%)$ wiesen ein sekundäres MDS auf. Daten zur Therapie lagen für 472 Patienten (64,7\%) vor. Bei 257 Patienten (35,3\%) wurde keine Therapie erfasst. Es wurden 1208 zytogenetische Untersuchungen mit 1 bis maximal 194 Metaphasen (Median 25 Metaphasen) durchgeführt. In 225 Fällen wurden zwischen zwei und zehn Folgeuntersuchungen erfasst, wobei bei 76 Patienten bereits bei Erstdiagnose eine klonale Evolution nachgewiesen werden konnte und bei 18 Patienten diese erst im Krankheitsverlauf identifiziert wurde. In Abbildung 3 ist die Häufigkeitsverteilung der MDS-Subgruppen gemäß der FAB-Klassifikation im Gesamtkollektiv der in der Studie erfassten Patienten bei Erstdiagnose dargestellt. Zusätzlich wurde eine Gruppe von Patienten erfasst, bei denen im Rahmen ihrer myelodysplastischen Erkrankung eine akute Leukämie (sekundäre Leukämie) diagnostiziert wurde. Eine Einteilung nach der WHO-Klassifikation war für die Patienten mit älteren Untersuchungszeitpunkten nicht möglich, da diese Klassifikation erst 2001 eingeführt wurde.

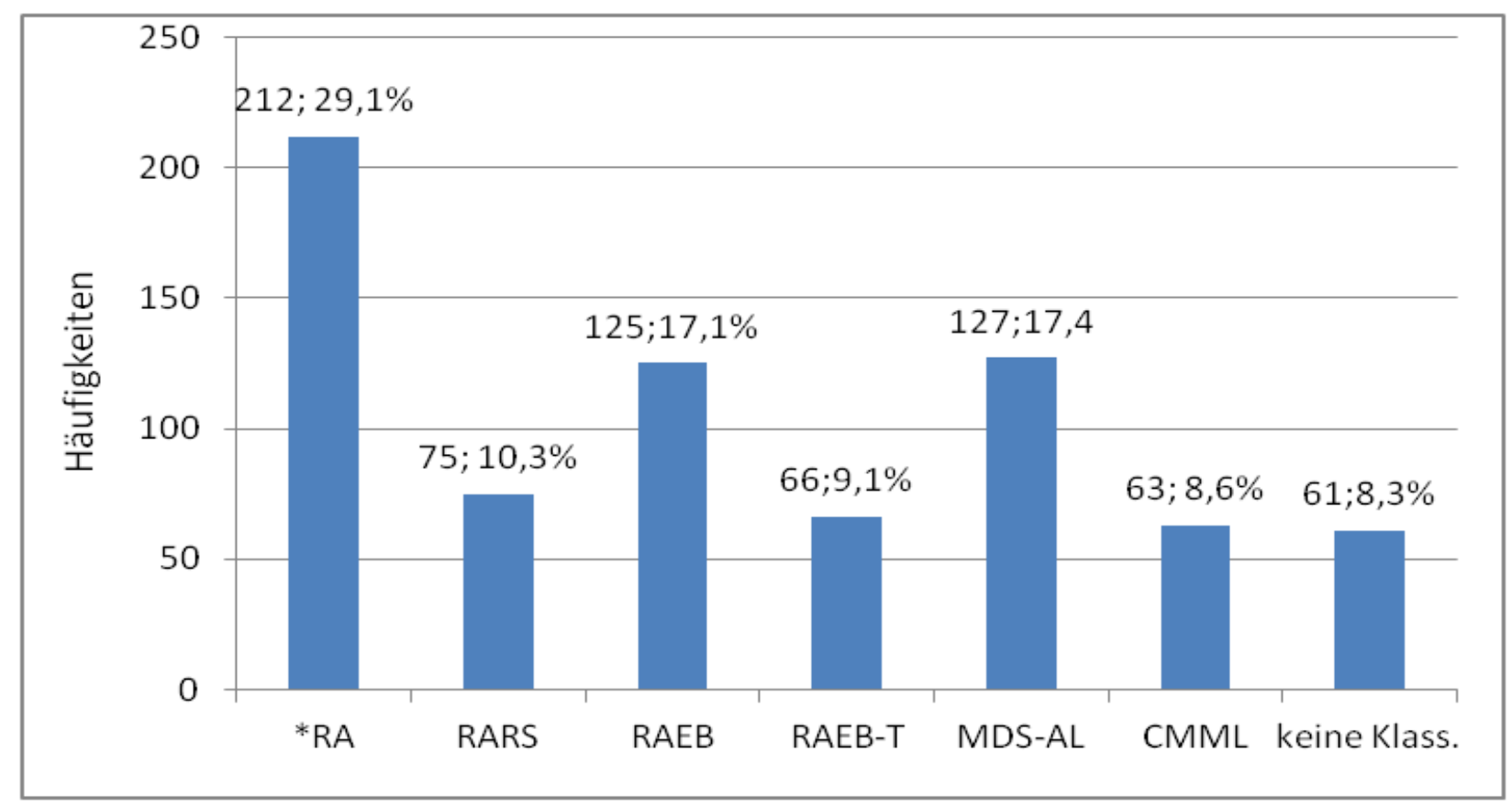

\section{Abbildung 3: Häufigkeitsverteilung der MDS-Subgruppen gemäß der FAB-Klassifikation im Gesamt- kollektiv bei Erstdiagnose}

${ }^{*} \mathrm{RA}=$ Refraktäre Anämie; RARS= Refraktäre Anämie mit Ringsideroblasten; RAEB= Refraktäre Anämie mit Exzess von Blasten; RAEB-T= Refraktäre Anämie mit Exzess von Blasten in Transformation; MDS-AL= Akute Leukämie nach Myelodysplastischem Syndrom; CMML = Chronische Myelomonozytäre Leukämie 


\subsection{Definition zytogenetischer Befunde}

Nach internationaler Einkunft wird der Karyotyp gemäß ISCN erfasst und beschrieben. Eine aktuelle Version wurde 2013 veröffentlicht (Shaffer et al. 2013). Somit ist eine einheitliche Dokumentation des Karyoptyps gewährleistet.

> Klonale Chromosomenaberrationen: Nachweis von Strukturanomalien oder Vorkommen gleicher Chromosomen in wenigstens zwei Metaphasen oder der Verlust von Chromosomen in mindestens drei Metaphasen.

Komplexe Aberration: Nachweis von mindestens drei Chromosomenaberrationen.

KIonale Evolution: Nachweis von zusätzlichen Chromosomenanomalien bei bereits vorhandener primärer Aberration eines Zellklons.

Eine klonale Evolution kann bereits bei Erstdiagnose oder im Erkrankungsverlauf nachgewiesen werden, abhängig davon, ob zusätzliche Chromosomenaberrationen gleichzeitig mit der Ursprungszelle oder unabhängig von dieser im Krankheitsprogress vorkommen (Schanz 2003, Haase 2005, Haase 2008). Entsprechend erfolgte im Rahmen dieser Studie eine Unterteilung der Patienten mit Nachweis einer klonalen Evolution in zwei Gruppen.

Gruppe 1: klonale Evolution bei Erstdiagnose

Gruppe 2: klonale Evolution im Verlauf der Erkrankung

\subsubsection{Verteilungstyp und Klongröße}

Der semiquanitative Anteil aberranter Metaphasen wird durch den Verteilungstyps (VT) angegeben (Schanz 2003, Haase 2005). Gemäß der Publikation von Bloomfield et al. (1984) erfolgte eine Unterteilung in die unten aufgeführten Gruppen:

NN-Karyotyp (^ Klongröße 0\%): Ausschließlich normale Metaphasen

NA-Karyotyp (^ Klongröße 1 - 99\%): Normale und aberrante Metaphasen im Mosaik AA-Karyotyp (^ Klongröße 100\%): Ausschließlich aberrante Metaphasen

Eine genauere Beurteilung über den Anteil aberranter Metaphasen erfolgt über die Bestimmung der Klongröße (Shaffer et al. 2013). Hierdurch ist eine quantitative Angabe möglich. Folgende Gruppeneinteilung für spätere Auswertungen wurde vorgenommen (Schanz 2003): 
Gruppe 1: Klongröße 1 - 20\%

Gruppe 2: Klongröße 21 - 50\%

Gruppe 3: Klongröße 51 - 80\%

Gruppe 4: Klongröße 81 - 99\%

Gruppe 5: Klongröße 100\%

\subsubsection{Aberrationen pro Fall}

Durch die Berechnung der Aberrationen pro Fall (A/Fall) ist die Erfassung der Anzahl von Chromosomenanomalien pro Fall exakt darstellbar. Die Methode wurde 1986 von Heim und Mitelman publiziert. Generell wurde der maximal komplexeste Klon zur Berechnung der Aberrationen pro Fall herangezogen.

\subsubsection{Theoretische klonale Evolutionsmuster}

Angelehnt an die publizierte Studie von Tricot et al. (1985) erfolgte eine erweiterte, modifizierte Kategorisierung verschiedener klonaler Evolutionsmuster durch die Arbeitsgruppe von Herrn Prof. Dr. med. D. Haase (Laborleitung Diagnostiklabore der Klinik für Hämatologie und Medizinische Onkologie, UMG) und damit auch eine Einteilung in unterschiedliche klinische Verläufe der MDS. Im Rahmen dieser Arbeit wurden fünf relevante Hauptgruppen mit jeweils mehreren Untergruppen gebildet. Die Zuordnung in die Gruppe „Genetische Progression“ erfolgte beim Nachweis des Auftretens einer Aberration bei initial normalem Karyotyp. Patienten mit zusätzlich auftretenden Anomalien bei primär aberrantem Karyotyp wurden ebenfalls dieser Gruppe zugeordnet. Die Größenzunahme des Klons um $>20 \%$ oder der Übergang in einen komplett aberranten Klon bei initialem Mosaik-Karyotyp (Steidl et al. 2005) wurden ebenfalls in der Kategorie „Genetische Progression“ erfasst. Patienten mit unverändert normalem oder aberrantem Karyotyp wurden in der Gruppe „Genetische Stabilität“ subsummiert. Patienten, die im Verlauf ihrer Erkrankung intermittierend einen normalen Karyotyp aufwiesen, wurden ebenfalls als genetisch stabil angesehen. In der Kategorie „Genetische Regression“ wurden Patienten mit primär aberrantem Karyotyp, bei denen im Krankheitsverlauf eine relevante Verkleinerung des Klons (>20\%) oder ein normaler Karyotyp nachgewiesen werden konnte, zusammengefasst. Die Zuordnung der Patienten in die Kategorie „Primäre Karyotypevolution“ erfolgte bei Nachweis von zusätzlich veränderten Metaphasen bei Erstdiagnose, die sich auf eine veränderte Ursprungszelle zurückführen ließen und mit dieser simultan auftraten (Schanz 
2003, Haase 2005, Haase 2008). Wechselnde transiente Klone sowie wechselnde Klongrößen wurden der Kategorie „Undulierenden Verlauf“ zugeordnet. Die unten aufgeführte Liste zeigt einen Überblick der beschriebenen Gruppen.

\section{A Genetische Progression:}

primär normal, im Verlauf aberrant

primär Mosaik, im Verlauf komplett aberrant

primär aberrant, Klon wird größer (>20\%)

primär aberrant, im Verlauf Zusatzanomalie

\section{B Genetische Stabilität:}

unverändert normal

unverändert aberrant

primär aberrant zwischendurch normaler Karyotyp

$$
\begin{aligned}
N & >A \\
N A & >A A \\
a N & >A n \\
A & >A+B
\end{aligned}
$$

\section{Genetische Regression:}

primär aberrant, im Verlauf normal

$$
A>N
$$

primär aberrant, Klon wird kleiner (<20\%)

An $>$ aN

D Primäre Karyotypevolution

$$
A / A+B / A+C
$$

\section{E Undulierend}

wechselnde transiente Klone

$\mathrm{A}>\mathrm{B}>\mathrm{C}$ 


\subsection{Klinische Daten}

Die klinischen Daten wurden sowohl bei der Erstdiagnose als auch im Verlauf zum Zeitpunkt der zytogenetischen Untersuchungen erfasst.

\section{Hämoglobin}

Der Hämoglobinwert $(\mathrm{Hb})$ wurde in g/dl angegeben. Für spätere Analysen erfolgte analog dem IPSS die Einteilung in folgende Gruppen (Greenberg et al. 1997):

Gruppe 0: Hb $\geq 10 \mathrm{~g} / \mathrm{dl}$

Gruppe 1: $\mathrm{Hb}<10 \mathrm{~g} / \mathrm{dl}$

\section{Leukozyten}

Die Anzahl der Leukozten im peripheren Blut wurde in * $10^{3} / \mu$ l angegeben und in folgenden zwei Gruppen unterschieden:

Gruppe 0: Leukozyten $\geq 1,8 * 10^{3} / \mu l$

Gruppe 1: Leukozyten $<1,8 * 10^{3} / \mu l$

\section{$>$ Neutrophile}

Die neutrophilen Granulozyten wurden als prozentualer Anteil der Leukozyten erfasst. Mit Hilfe der Leukozytenzahl wurde dann der Absolutwert (ANC) der neutrophilen Granulozyten berechnet. Die Angabe erfolgte in ${ }^{*} 10^{3} / \mu l$. Eine Aufteilung für nachfolgende Auswertungen war nicht erforderlich.

\section{Thrombozyten}

Die Anzahl der Thrombozyten wurde in * $10^{3} / \mu$ l erfasst und nach der Höhe der Werte in zwei Gruppen aufgeteilt (Greenberg et al. 1997):

Gruppe 0: Thrombozyten $\geq 100 * 10^{3} / \mu \mathrm{l}$

Gruppe 1: Thrombozyten $<100^{*} 10^{3} / \mu \mathrm{l}$

\section{Blasten im Knochenmark}

Die Blasten im Knochenmark wurden ebenfalls in Prozent (\%) erfasst und die Einteilung in Gruppen erfolgte anhand der Höhe des Blastenanteils entsprechend der Publikation von Schanz et al. (2011) mit einer zusätzlichen Gruppe (Gruppe 5): 
Gruppe 1: $\quad<5 \%$

Gruppe 2: $\quad 5-10 \%$

Gruppe 3: $\quad 11-20 \%$

Gruppe 4: $\quad 21-30 \%$

Gruppe 5: $\quad>30 \%$

\subsection{Statistische Methoden}

Die statistische Auswertung erfolgte computergestützt mit dem Statistikprogramm SPSS Version 20 (IBM Corp. IBM SPSS Statistics for Windows, Armonk, New York).

Zur Schätzung der Überlebenswahrscheinlichkeit wurde die von Kaplan und Meier (1958) beschriebene Methode angewendet und zur Signifikanzberechnung für die Gruppenvergleiche wurde der Log-Rank-Test (Peto et al. 1977) verwendet. Sowohl Patienten, die am Ende des Beobachtungszeitraumes noch lebten, als auch Patienten mit „Iost to follow up“, wurden zensiert.

Unterschiede zwischen qualitativen Variablen wurden mittels des Chi-Quadrat-Tests nach Pearson überprüft.

Bei nicht normalverteilten Stichproben wurden zur Signifikanzberechnung nichtparametrische Tests angewendet. Bei unabhängigen Stichproben erfolgte die Berechnung mit dem Mann-Whitney-U-Test (M-W-U-Test). Bei gepaarten Stichproben wurde der WilcoxonVorzeichen-Rang-Test (Wilcoxon-Test) angewandet.

Zur Berechnung von multivariaten Analysen wurde das „proportional-hazardRegressionsmodell“ verwendet (Cox 1972).

Als gängige Grenzen wurden die Unterschiede bei $p$-Werten $<0,05$ als signifikant und Unterschiede bei $p-W e r t e n$ von $<0,01$ als hochsignifikant gewertet. 


\section{Ergebnisse}

\subsection{Die klonale Evolution}

\subsubsection{Häufigkeit in der Gesamtgruppe}

Innerhalb der Gesamtgruppe trat die klonale Evolution mit einer Häufigkeit von 12,9\% $(n=94)$ auf. Betrachtete man aber nur die aberranten Fälle, lag der Anteil der klonalen Evolution bei 25,8\%. In den folgenden Abschnitten wird die klonale Evolution in den einzelnen Subgruppen dargestellt.

\subsubsection{Geschlecht}

Bei 16\% ( $n=48)$ aller zytogenetisch untersuchten Frauen und 10,7\% $(n=46)$ aller Männer wurde eine klonale Evolution nachgewiesen ( $p=0,037)$. Somit trat bei den Frauen des Patientenkollektivs eine klonale Evolution signifikant häufiger auf als bei den Männern (Abbildung 4).

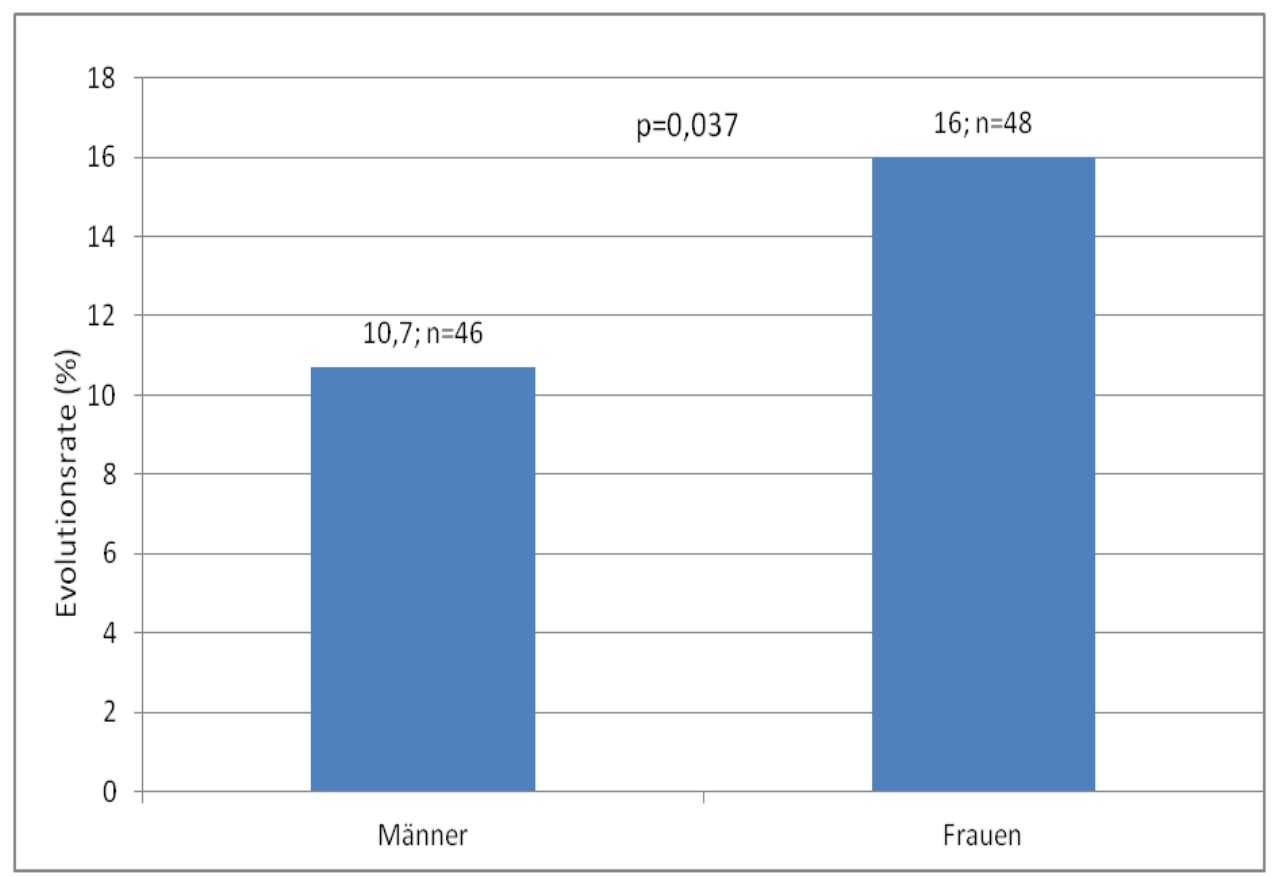

\section{Abbildung 4: Klonale Evolution nach Geschlecht}

\subsubsection{Altersgruppe}

Hinsichtlich des Auftretens einer klonalen Evolution in den definierten Altersgruppen (AG1: < 16 Jahre; AG2: 16 - 60 Jahre; AG3: > 60 Jahre, Schanz 2003, Haase 2005) konnten keine signifikanten Unterschiede festgestellt werden. Allerdings zeigte sich, dass die Häufigkeit der klonalen Evolution mit steigendem Alter zunimmt (Schanz 2003) (Tabelle 5). 
Ergebnisse

Tabelle 5: Häufigkeit klonaler Evolution in den Altersgruppen

\begin{tabular}{lccc}
\hline & Evolution (n) & Evolution (\%) & p-Wert \\
\hline AG1 (< 16 Jahre) & 2 & 7,1 & \\
\cline { 1 - 3 } AG2 (16-60 Jahre) & 27 & 12,0 & \multirow{2}{*}{0,417} \\
\cline { 1 - 3 } AG3 (>60 Jahre) & 64 & 13,8 & \\
\hline
\end{tabular}

\subsubsection{3 Ätiologie}

Bei 12,7\% ( $n=84)$ der Patienten mit primärem MDS und 15,2\% $(n=10)$ der Patienten mit sekundärem MDS konnte das Auftreten einer klonalen Evolution gefunden werden. Mit einem p-Wert von 0,566 konnte kein signifikanter Unterschied im Auftreten der klonalen Evolution zwischen diesen Gruppen festgestellt werden (Abbildung 5).

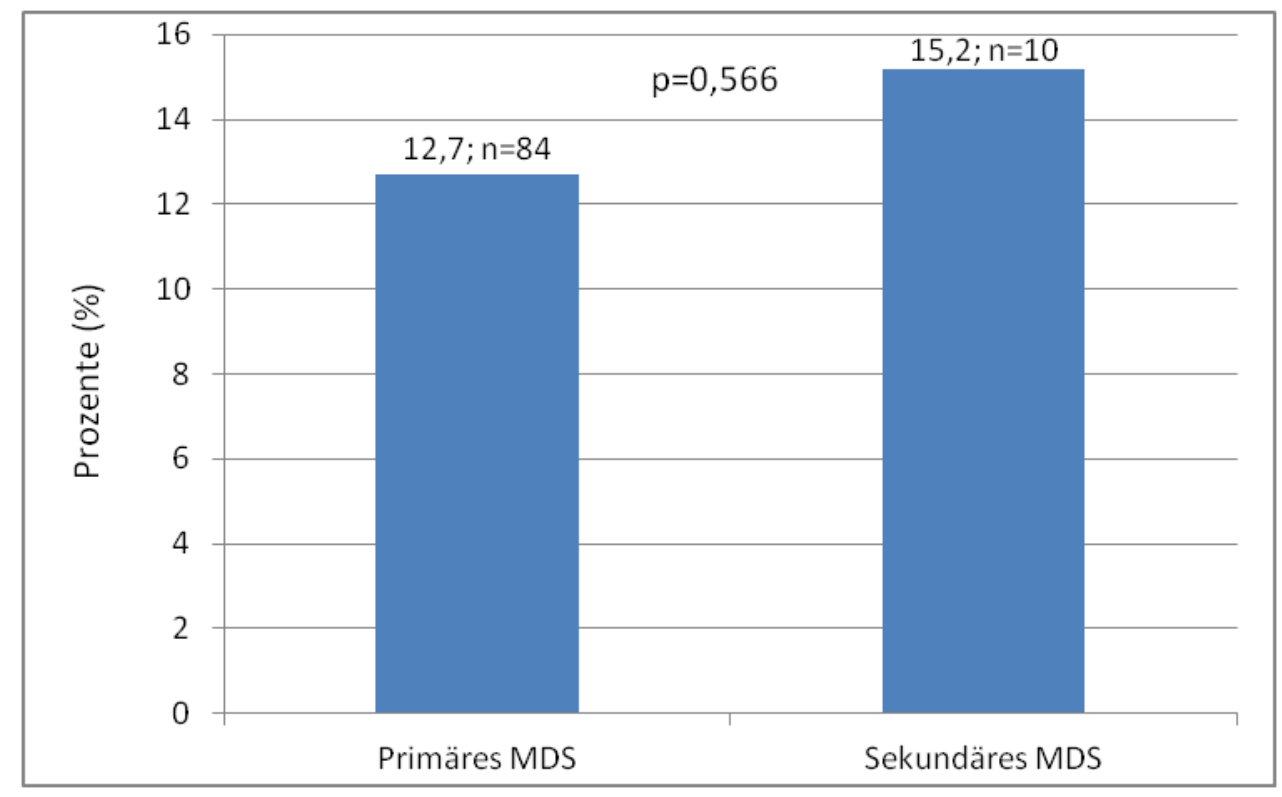

Abbildung 5: Häufigkeit klonaler Evolution abhängig von der Ätiologie

\subsection{Klinische Daten}

\subsubsection{Gesamtgruppe zum Zeitpunkt der Erstdiagnose}

Die Darstellung der hämatologischen Parameter und des Blastenanteils erfolgte für Patienten mit und ohne klonale Evolution sowie für das Gesamtkollektiv. Von 729 Patienten im Gesamtkollektiv konnte in 538 Fällen der Blastenanteil im Knochenmark (KM) eruiert werden. Hiervon waren 72 Patienten der Gruppe mit klonaler Evolution und 466 Patienten der Gruppe ohne klonale Evolution. In 191 Fällen konnten keine Daten erfasst werden. Ebenfalls konnten die Daten für die hämatologischen Parameter Hämoglobin, Leukozyten, ab- 
solute Neutrophilenzahl (ANC) sowie Thrombozyten nicht vollständig erhoben werden. Die gültige Anzahl an Patienten, bei denen eine Auswertung durchgeführt werden konnte, ist in der Tabelle 6 im Detail dargestellt.

Tabelle 6: Klinische Daten der Gesamtgruppe bei Erstdiagnose

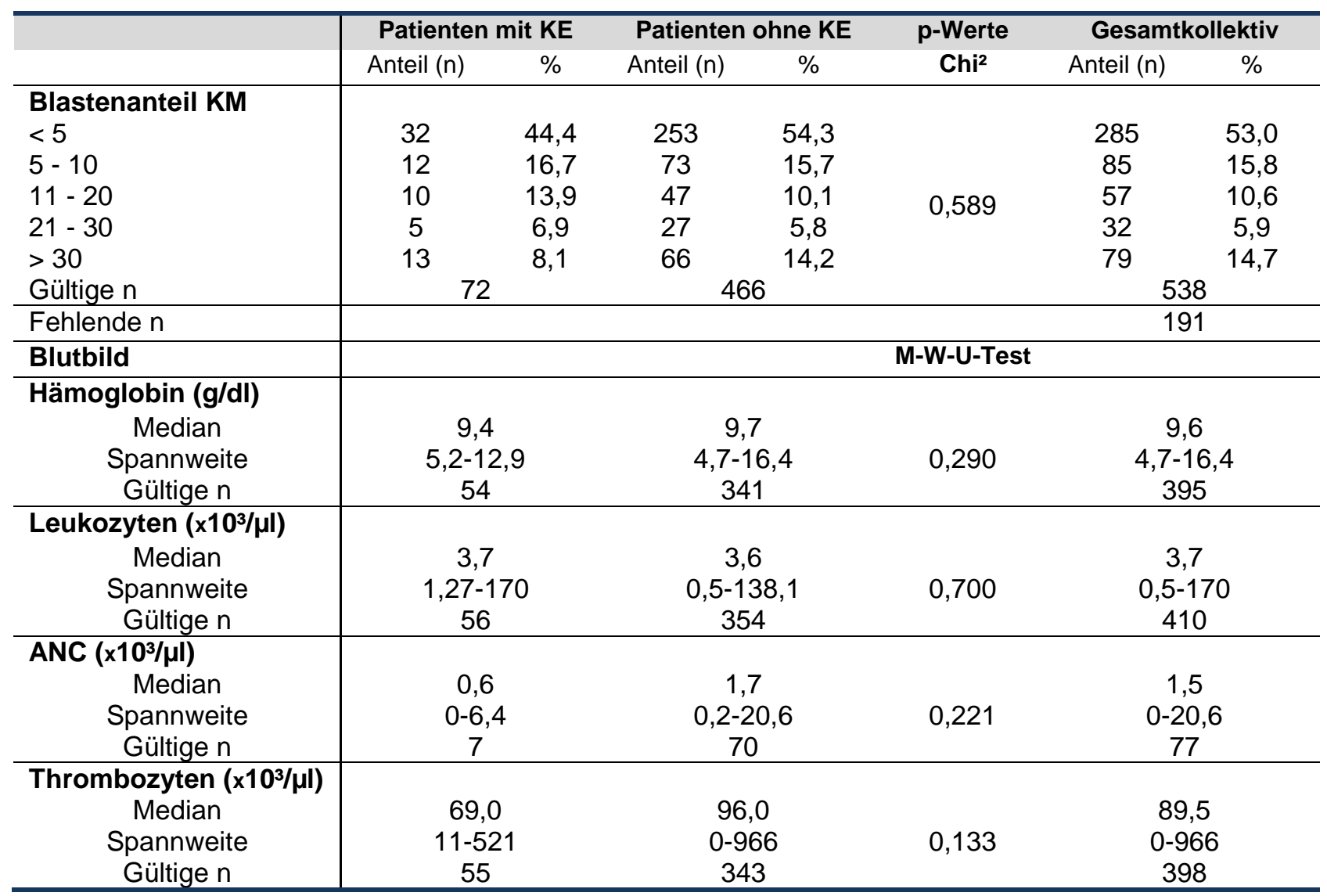

Im Vergleich beider Gruppen (Patienten mit vs. ohne klonale Evolution (KE)) zeigte sich, dass die Neutrophilen- und Thrombozytenwerte im Median bei Patienten mit klonaler Evolution deutlich niedriger waren. Ein signifikantes Niveau konnte jedoch nicht erreicht werden. Hinsichtlich der übrigen klinischen Parameter konnten ebenfalls keine signifikanten Unterschiede festgestellt werden (Tabelle 6).

\subsubsection{Vergleich der klinischen Daten}

\subsubsection{Patienten ohne klonale Evolution mit Verlaufsdaten}

In 186 Fällen konnten bei Patienten ohne Nachweis einer klonalen Evolution zytogenetische Verlaufsdaten erhoben werden. In Tabelle 7 sind die klinischen Daten dieser Patienten zum Zeitpunkt der Erstdiagnose und am Ende der Beobachtungszeit aufgeführt, wobei die klinischen Daten nicht immer lückenlos erfasst werden konnten. Die Daten zum Blastenanteil im Knochenmark (KM) wurden in 164 Fällen bei Erstdiagnose und in 
121 Fällen am Ende der Beobachtungszeit dokumentiert. Ebenfalls konnten die Parameter für die übrigen klinischen Daten (Hämoglobin, Leukozyten, absolute Neutrophilenzahl (ANC) sowie Thrombozyten) nicht vollständig erhoben werden. Die Anzahl der hier ausgewerteten Patienten ist in Tabelle 7 im Detail dargestellt. Die mediane Beobachtungszeit betrug 13,9 Monate mit einem Minimum von 0,2 und einem Maximum von 117,6 Monaten.

Der Vergleich der klinischen Daten zeigte einen signifikanten Anstieg der Neutrophilenwerte zum Ende der Beobachtung $(p<0,01)$. Die Thrombozytenwerte waren zum Ende der Beobachtung mit einem Median von $77,1 \times 10^{3} / \mu \mathrm{l}$ signifikant niedriger als zum Zeitpunkt der Erstdiagnose $(p=0,015)$. Hinsichtlich der übrigen klinischen Parameter fanden sich keine signifikanten Differenzen im Vergleich zu den Ausgangswerten. Sowohl der Blastenanteil im Knochenmark als auch der Hämoglobinwert und die Leukozytenzahl lagen zum Zeitpunkt der Erstdiagnose und am Ende der Beobachtung nahe beieinander.

Tabelle 7: Klinische Daten der Patienten ohne klonale Evolution mit Verlaufsdaten

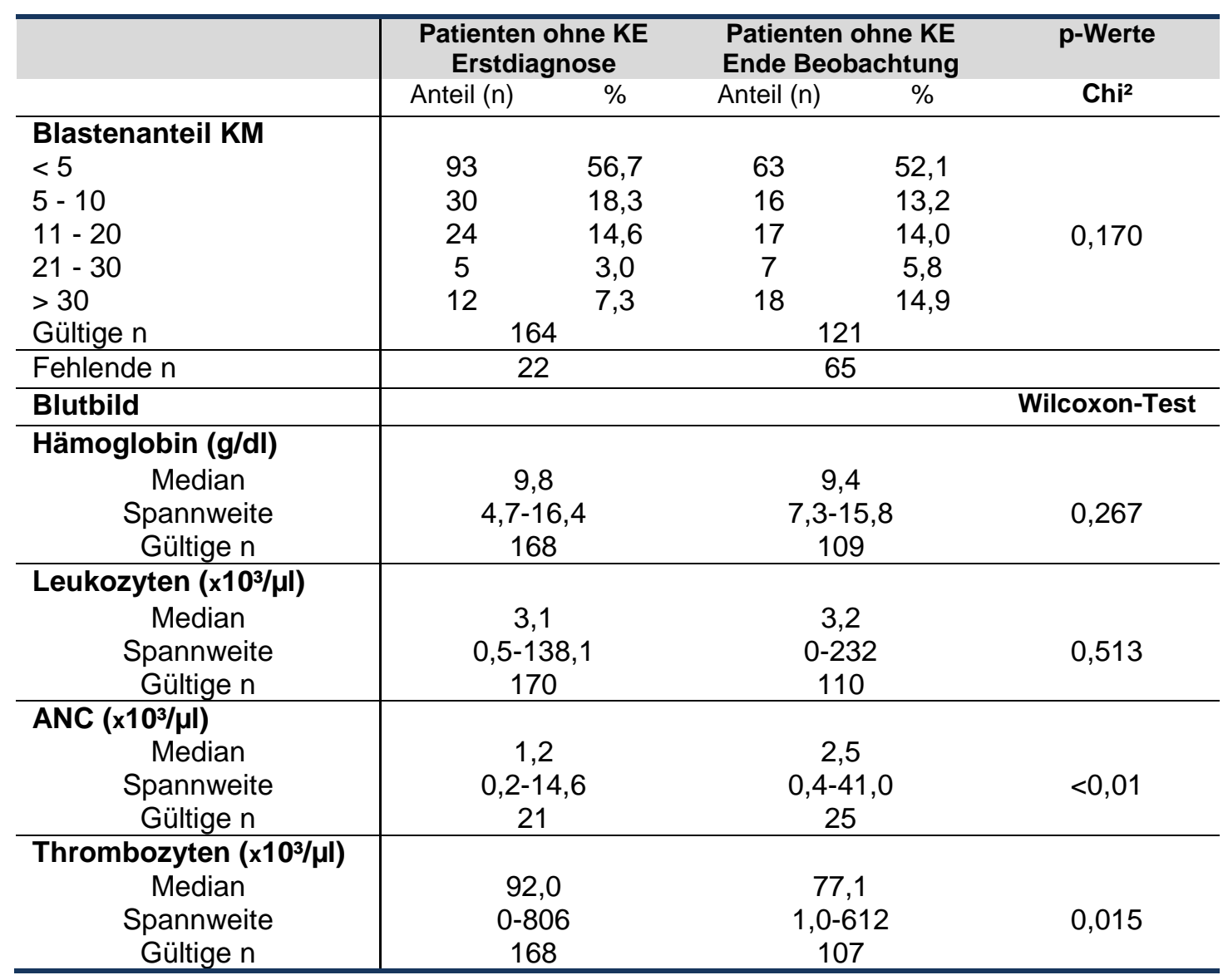




\subsubsection{Patienten mit klonaler Evolution}

Dargestellt sind die klinischen Daten der Patienten, bei denen bereits bei der Erstdiagnose eine klonale Evolution („Primäre Karyotypevolution“) nachgewiesen wurde (Tabelle 8). Auch hier wurden die prognostisch relevanten klinischen Daten zum Zeitpunkt der Erstdiagnose und zum Ende des Beobachtungszeitraumes ausgewertet. Bei 76 Patienten konnte bereits bei Erstdiagnose eine klonale Evolution nachgewiesen werden. Die klinischen Daten konnten nicht lückenlos erfasst werden. Es wurden in 57 der Fälle Daten zum Blastenanteil im Knochenmark (KM) bei Erstdiagnose dokumentiert. In 21 von 76 Fällen wurden Verlaufsdaten erfasst. Hiervon konnten in 10 Fällen Daten zum Blastenanteil im Knochenmark (KM) am Ende der Beobachtungszeit ausgewertet werden. In 11 der 21 Fälle konnten die Daten nicht eruiert werden. Ebenfalls zeigten sich in der Auswertung der übrigen hämatologischen Parameter (Hämoglobin, Leukozyten, absolute Neutrophilenzahl (ANC) sowie Thrombozyten) Diskrepanzen. Die Anzahl der ausgewerteten Patienten ist in Tabelle 8 im Einzelnen dargestellt. Die mediane Beobachtungszeit betrug 5,8 Monate mit einem Minimum von 0,2 und ein Maximum von 38,7 Monaten.

Die Patienten hatten am Ende des Beobachtungszeitraumes signifikant niedrigere Hämoglobinwerte als bei Erstdiagnose $(p<0,01)$. Auch die weiteren Laborparameter wiesen zum Ende des Beobachtungszeitraumes eine Tendenz zu niedrigeren Werten auf. Diese Differenzen zum jeweiligen Ausgangswert erreichten jedoch kein signifikantes Niveau, möglicherweise bedingt durch die geringen Fallzahlen. Der $p$-Wert für die Änderung der Neutrophilenwerte konnte wegen kleiner Fallzahl nicht berechnet werden. 
Ergebnisse

Tabelle 8: Klinische Daten der Patienten mit klonaler Evolution bei Erstdiagnose

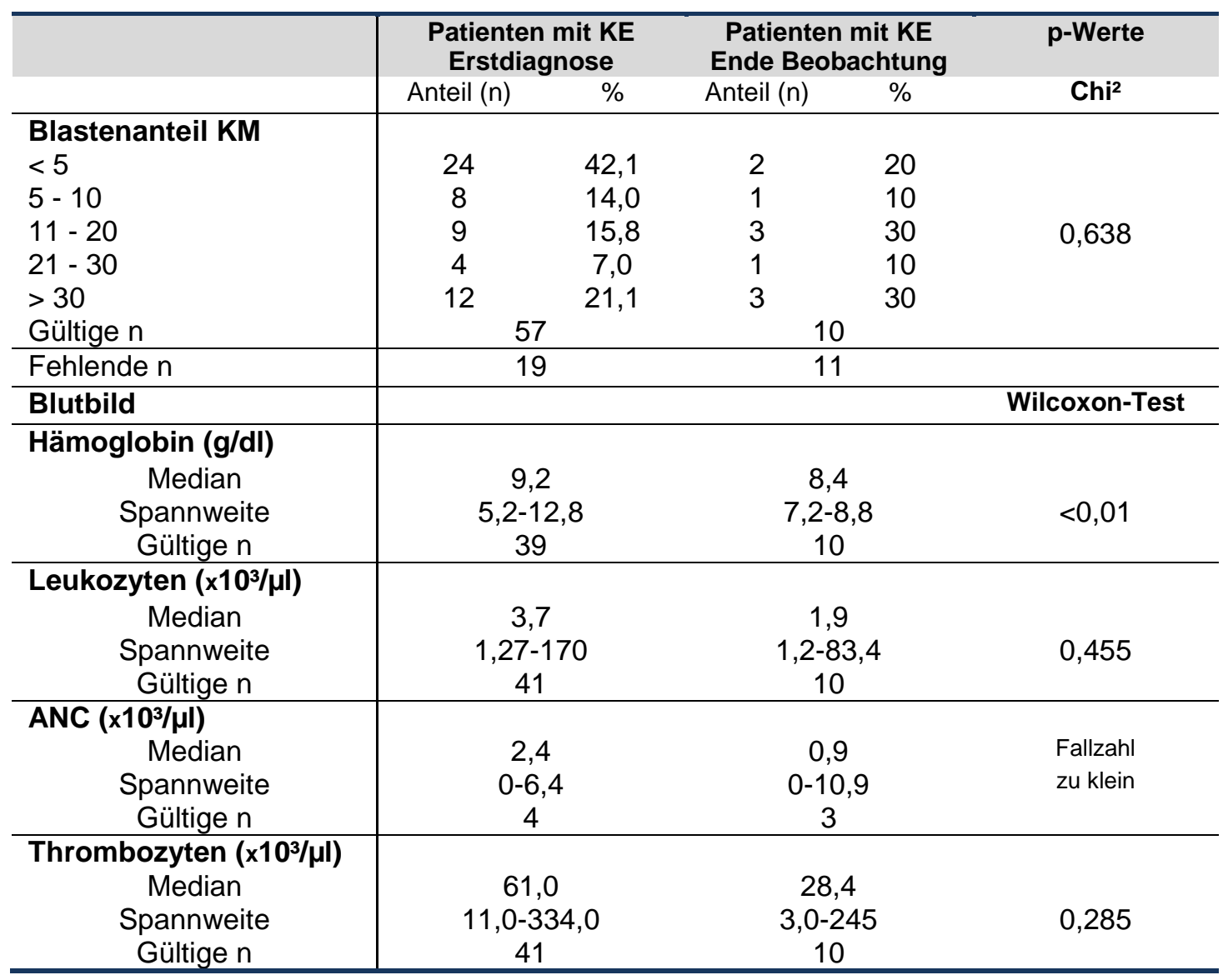

\subsubsection{Patienten mit klonaler Evolution im Krankheitsverlauf}

Die klinischen Daten der 18 Patienten, bei denen im Krankheitsverlauf eine klonale Evolution nachgewiesen werden konnte, wurden auch hier entsprechend miteinander verglichen (Tabelle 9). Zum einen wurden die klinischen Parameter zum Ende des Beobachtungspunkts und zum anderen bei zytogenetischem Nachweis klonaler Evolution mit den klinischen Daten bei Erstdiagnose verglichen. Zum Zeitpunkt der Erstdiagnose eines MDS konnten in 15 der 18 Fälle Daten zum Blastenanteil im Knochenmark (KM) erfasst werden. Im Weiteren wurden in 11 Fällen Daten zum Blastenanteil im Knochenmark (KM) bei Nachweis einer klonalen Evolution im Krankheitsverlauf erhoben. Ebenfalls konnten Daten zum Blastenanteil im Knochenmark (KM) in weiteren 11 Fällen am Ende der Beobachtungszeit erfasst werden. Aufgrund der lückenhaften Datenerhebung konnten nicht alle Daten für die hämatologischen Parameter Hämoglobin, Leukozyten, absolute Neutrophilenzahl (ANC) sowie Thrombozyten vollständig erfasst werden. Die Anzahl der Patienten, die zur Auswertung herangezogen werden konnten, sind in der Tabelle $9 \mathrm{im}$ Detail dargestellt. Die mediane Beobachtungszeit bis zum Nachweis einer klonalen Evolution betrug 11,9 Monate mit einem Minimum von 2,8 und einem Maximum von 46,3 Monaten. 
Der Anteil der Blasten im Knochenmark am Ende des Beobachtungszeitraumes lag im Vergleich zur Erstdiagnose signifikant höher $(p=0,029)$. Auch zum Zeitpunkt des Nachweises einer klonalen Evolution fanden sich im Vergleich zur Erstdiagnose höhere Blastenanteile. Ein signifikanter Unterschied konnte hier jedoch nicht festgestellt werden $(p=0,069)$.

Ein weiterer Unterschied konnte bezüglich der Thrombozytenwerte erfasst werden. Es fand sich ein deutlicher Abfall der Thrombozytenzahl zum Ende des Beobachtungszeitraumes im Vergleich zum Ausgangswert $(p=0,012)$. Ebenfalls zeigten die Leukozyten- und Neutrophilenzahlen im Verlauf der Beobachtung eine abnehmende Tendenz.

Tabelle 9: Klinische Daten der Patienten mit klonaler Evolution im Krankheitsverlauf

\begin{tabular}{|c|c|c|c|c|c|c|c|}
\hline & \multicolumn{7}{|c|}{ Patienten mit klonaler Evoluiton im Krankheitsverlauf } \\
\hline & Bei Erstdiagnos & Ende Beobacht & ung & $\mathrm{p}$-Werte & Bei Nachweis v & von $\mathrm{KE}$ & p-Wert \\
\hline & Anteil (n) $\%$ & Anteil (n) & $\%$ & $\mathrm{Chi}^{2}$ & Anteil (n) & $\%$ & $\mathrm{Chi}^{2}$ \\
\hline $\begin{array}{l}\text { Blastenanteil KM } \\
<5 \\
5-10 \\
11-20 \\
21-30 \\
>30 \\
\text { Gültige } n\end{array}$ & 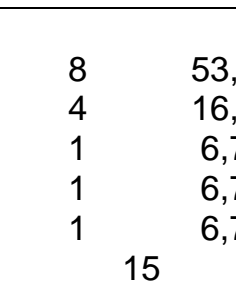 & $\begin{array}{ll}3 & \\
2 & \\
1 & \\
5 & \\
& 11\end{array}$ & $\begin{array}{c}27,3 \\
18,2 \\
9,1 \\
45,5\end{array}$ & 0,029 & $\begin{array}{ll}1 & \\
2 & \\
2 & \\
1 & \\
5 & \\
& 11\end{array}$ & $\begin{array}{c}9,1 \\
18,2 \\
18,2 \\
9,1 \\
45,5\end{array}$ & 0,069 \\
\hline Fehlende $\mathrm{n}$ & 3 & 7 & & & 7 & & \\
\hline Blutbild & & & & $\begin{array}{c}\text { Wilcoxon- } \\
\text { Test }\end{array}$ & & & $\begin{array}{c}\text { Wilcoxon } \\
\text { Test }\end{array}$ \\
\hline $\begin{array}{c}\text { Hämoglobin }(\mathbf{g} / \mathbf{d l}) \\
\text { Median } \\
\text { Spannweite } \\
\text { Gültige n }\end{array}$ & $\begin{array}{c}9,6 \\
6,1-12,9 \\
15\end{array}$ & $\begin{array}{c}9,4 \\
8,4-10,2 \\
10\end{array}$ & & 0,343 & $\begin{array}{c}9,4 \\
8,4-10,2 \\
10\end{array}$ & & 0,441 \\
\hline $\begin{array}{c}\text { Leukozyten }\left(x^{10} / \mu l\right) \\
\text { Median } \\
\text { Spannweite } \\
\text { Gültige n } \\
\end{array}$ & $\begin{array}{c}3,7 \\
1,4-31,3 \\
15 \\
\end{array}$ & $\begin{array}{c}1,7 \\
0,4-10,6 \\
10 \\
\end{array}$ & & 0,441 & $\begin{array}{c}1,7 \\
0,4-10,6 \\
10 \\
\end{array}$ & & 0,441 \\
\hline $\begin{array}{c}\text { ANC }\left(\mathbf{x} 10^{3} / \mu \mathrm{l}\right) \\
\text { Median } \\
\text { Spannweite } \\
\text { Gültige } n\end{array}$ & $\begin{array}{c}0,4 \\
0,3-1,2 \\
3\end{array}$ & $\begin{array}{c}0,15 \\
0,1-0,2 \\
2\end{array}$ & & $\begin{array}{c}\text { Fallzahl zu } \\
\text { klein }\end{array}$ & $\begin{array}{c}0,2 \\
0,1-1,7 \\
3\end{array}$ & & 0,655 \\
\hline $\begin{array}{c}\text { Thrombozyten }\left(\mathbf{x} 10^{3} / \mu l\right) \\
\text { Median } \\
\text { Spannweite } \\
\text { Gültige } n\end{array}$ & $\begin{array}{c}118,5 \\
27-521 \\
14\end{array}$ & $\begin{array}{c}38,1 \\
11,5-213 \\
9\end{array}$ & & 0,012 & $\begin{array}{c}41,4 \\
11,5-25 \\
10\end{array}$ & & 0,086 \\
\hline
\end{tabular}

\subsubsection{Die Prognostische Relevanz der klonalen Evolution}

\subsubsection{Gesamtkollektiv}

Der Vergleich der Überlebenskurven für Patienten mit bzw. ohne klonale Evolution zeigte einen hochsignifikanten Unterschied (Abbildung 6). 
Bei 72 Patienten mit einer klonalen Evolution lagen Überlebensdaten vor; davon waren $51,4 \%(n=37)$ zensiert. Bei 421 Patienten ohne klonale Evolution konnten Überlebensdaten mit einer Rate von 70,3\% ( $n=296)$ zensierter Fälle ausgewertet werden. Patienten mit klonaler Evolution zeigten signifikant kürzere Überlebenszeiten und damit eine deutlich schlechtere Prognose. Die mediane Überlebenszeit betrug 24,4 Monate. Diese verlängerte sich bei Patienten ohne klonale Evolution auf 103,6 Monate $(p<0,01)$.

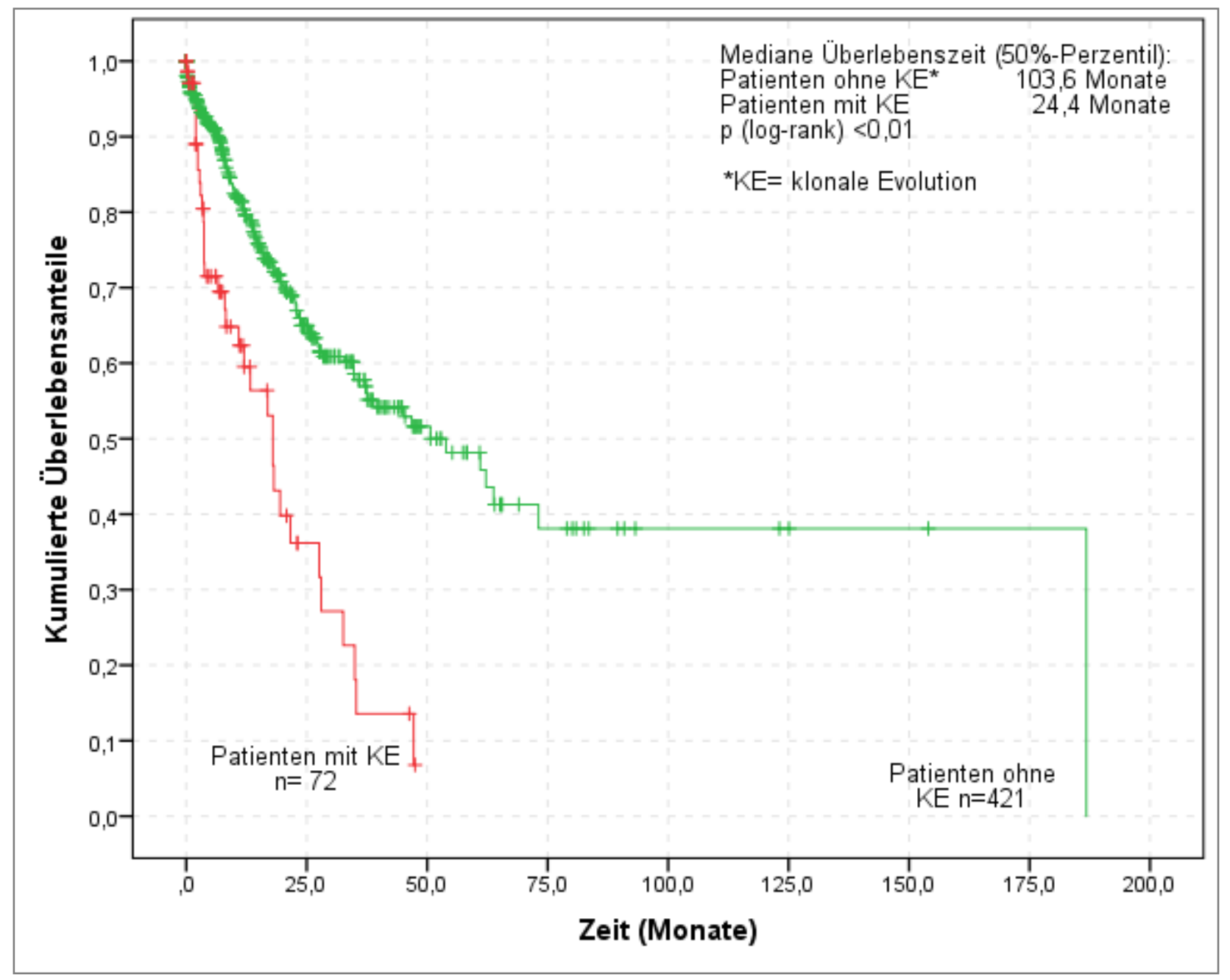

Abbildung 6: Kumulierte Überlebensanteile (Kaplan-Meier) der Gesamtgruppe

\subsubsection{Prognostische Relevanz der klonalen Evolution zum Diagnosezeitpunkt}

Untersucht wurden Patienten mit klonaler Evolution zum Zeitpunkt der Erstdiagnose und im Krankheitsverlauf (Abbildung 7). Bei 54 Patienten, bei denen bereits bei Erstdiagnose eine klonale Evolution diagnostiziert wurde, lagen Überlebensdaten vor. 48,1\% ( $n=26)$ dieser Daten wurden zensiert. In der zweiten Gruppe konnten bei 18 Patienten Überlebensdaten mit einer Rate von $61,1 \%(n=11)$ zensierter Fälle eruiert werden. Auch hier 
zeigten sich hochsignifikante Unterschiede im Überleben. Bei Nachweis einer klonalen Evolution bereits bei Erstdiagnose lag die mediane Überlebenszeit bei nur 8,2 Monaten. Diese verlängerte sich auf 32,6 Monate bei Nachweis einer klonalen Evolution im Verlauf der Erkrankung $(p<0,01)$.

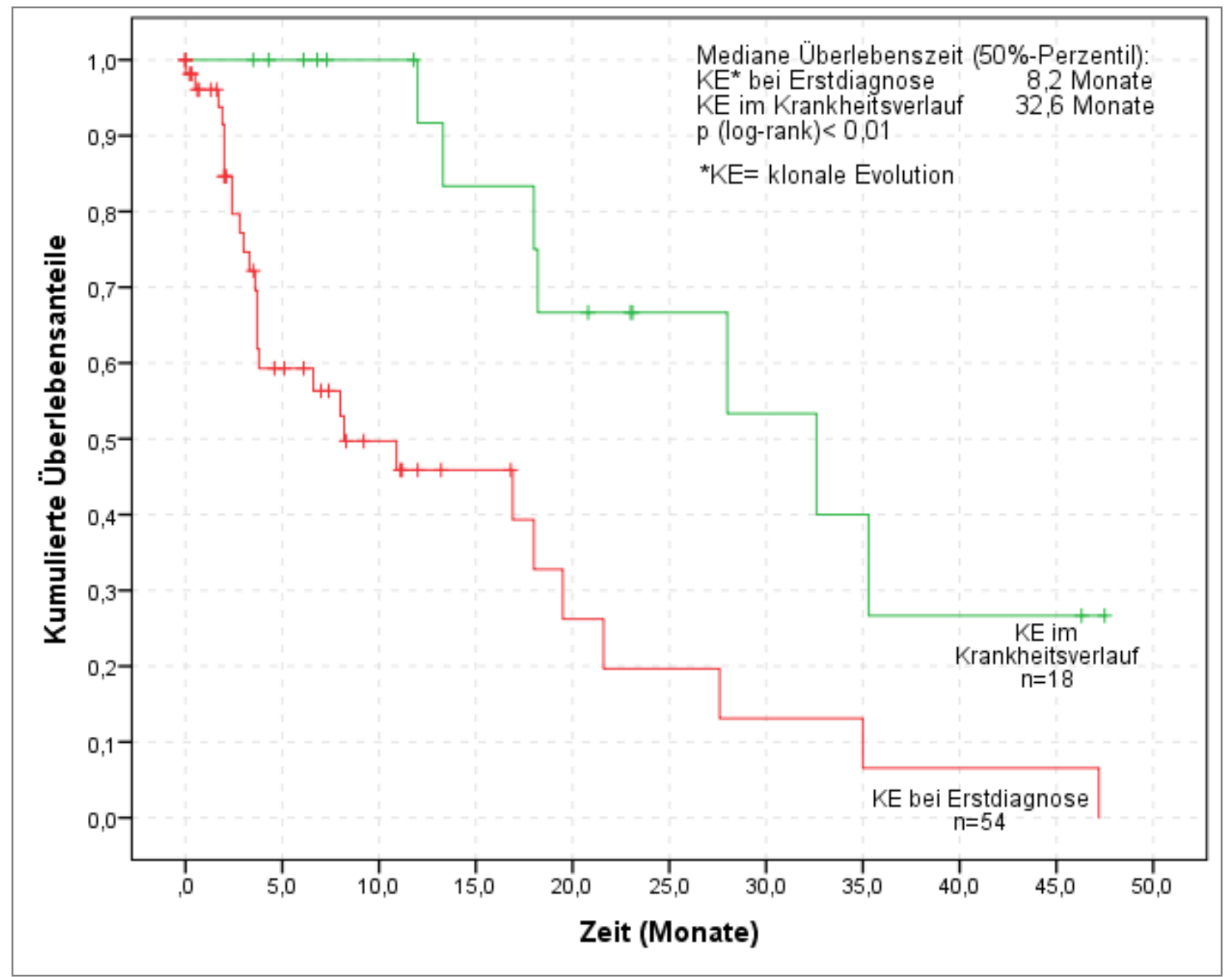

Abbildung 7: Kumulierte Überlebensanteile (Kaplan-Meier) in Abhängigkeit vom Diagnosezeitpunkt der klonalen Evolution

Patienten zum Zeitpunkt der Erstdiagnose wiesen, im Vergleich zu denen, die im Krankheitsverlauf eine klonale Evolution aufzeigten, eine höhere Anzahl an Aberrationen auf (Abbildung 8). Diese Patienten sind mutmaßlich bereits bei Erstdiagnose in ihrem Krankheitsprogress weiter fortgeschritten. Diese Annahme ist hypothetisch, da der biologische Entstehungszeitpunkt der Erkrankung nicht sicher eruierbar ist. 


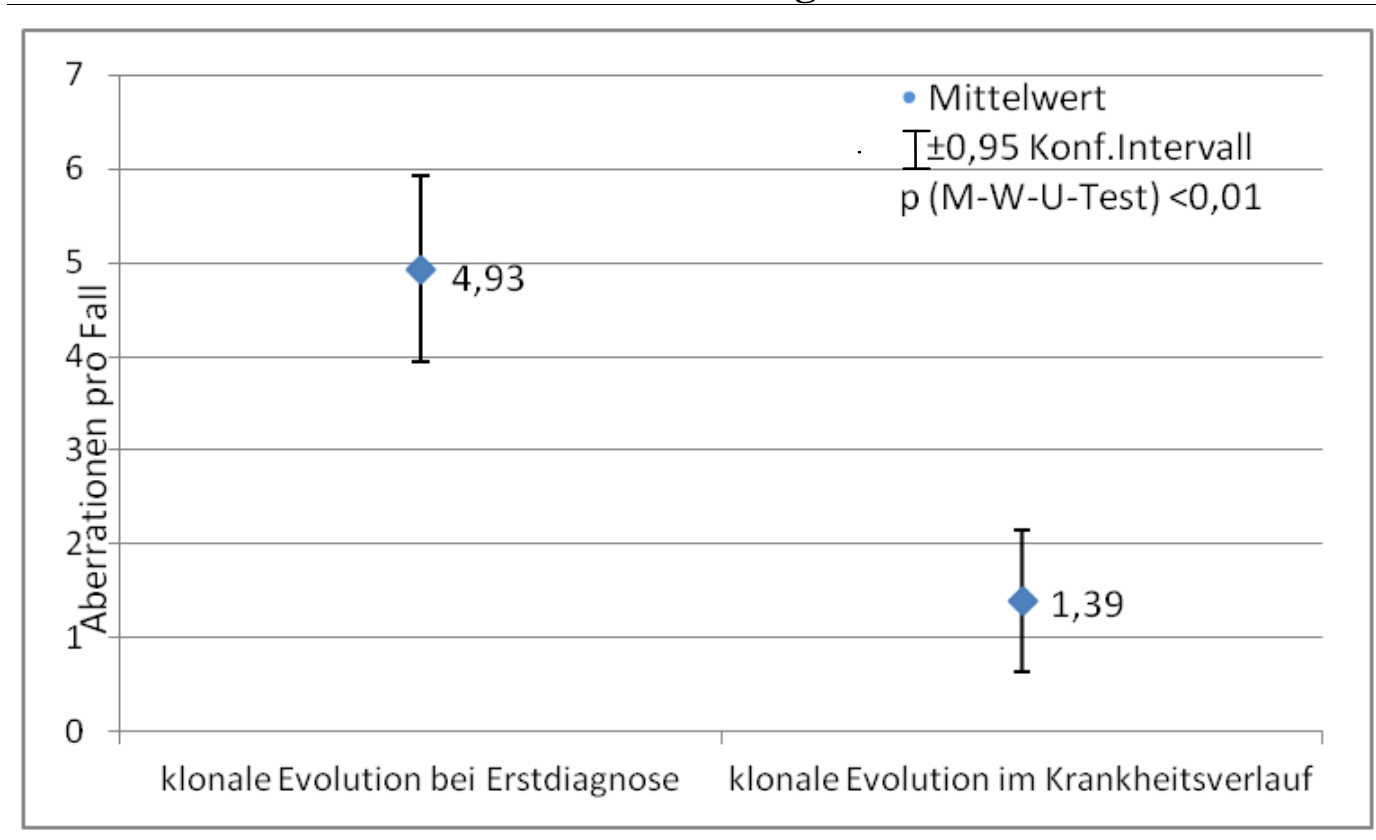

Abbildung 8: Aberrationen pro Fall in Abhängigkeit vom Diagnosezeitpunkt der klonalen Evolution

\subsubsection{Prognostische Relevanz des zeitlichen Auftretens der klonalen Evolution}

Es konnte bereits gezeigt werden, dass der Nachweis einer klonalen Evolution mit einer schlechten Prognose einhergeht (siehe 3.2.3.1). Unter Berücksichtigung der Tatsache, dass die klonale Evolution die genetische Instabilität und den Krankheitsprozess widerspiegelt (Haase 2005, Haase et al. 2011), erfolgte eine weitere Analyse hinsichtlich des zeitlichen Auftretens der klonalen Evolution (Abbildung 9). Die mediane Beobachtungszeit betrug 363 Tage. Darauf basierend wurden die Patienten, bei denen innerhalb von 365 Tagen nach Diagnosestellung eine klonale Evolution nachgewiesen wurde, in die Gruppe 1 (frühe klonale Evolution) und die Patienten, bei denen >365 Tage nach der Diagnosestellung eine klonale Evolution festgestellt wurde, in die Gruppe 2 (späte klonale Evolution) aufgeteilt.

Diese Aufteilung zeigte einen hochsignifikanten Unterschied im zeitlichen Verlauf für das Auftreten einer klonalen Evolution. In der Gruppe 1 wurde eine klonale Evolution im Median 5,3 Monate nach der Erstdiagnose festgestellt. Im Vergleich dazu war in der Gruppe 2 mit im Median 21,9 Monaten ein deutlich längeres Zeitintervall bis zum Nachweis der klonalen Evolution nachweisbar.

Anhand dieser Ergebnisse konnte ein Unterschied im Krankheitsprozess dargestellt werden. Die MDS-Zellen bei Patienten mit einer frühen Evolution sind wahrscheinlich genetisch instabiler. Es zeigt sich hier ein rasches Auftreten einer klonalen Evolution. In der Gruppe der späten klonalen Evolution zeigte sich das Auftreten der Evolution dagegen 
verzögert, was möglicherweise durch eine schrittweise Akkumulation genetischer Aberrationen erklärbar ist (Haase 2005, Haase 2008).

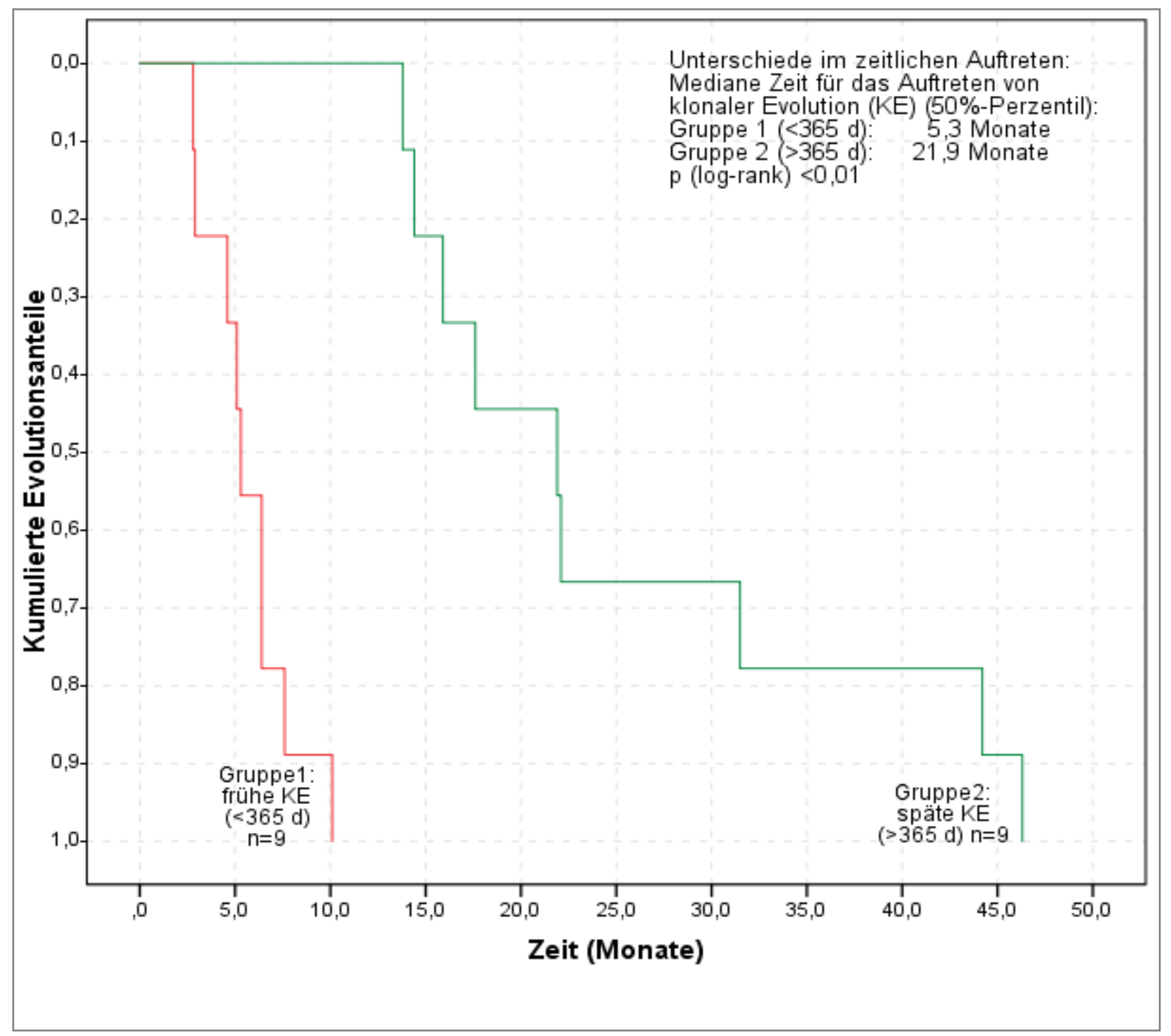

Abbildung 9: Auftreten klonaler Evolution im zeitlichen Verlauf: Frühe und späte klonale Evolution

Neben der Darstellung des zeitlichen Auftretens der klonalen Evolution (Abbildung 9), konnten in einer weiteren Untersuchung Unterschiede in den medianen Überlebenszeiten der beiden Gruppen festgestellt werden (Abbildung 10). Das Auftreten einer klonalen Evolution innerhalb von 365 Tagen (frühe klonale Evolution; Gruppe 1) nach Diagnosestellung ist prognostisch eher ungünstig. Die mediane Überlebenszeit betrug 13,25 Monate ( $n=9$, zensiert: $n=7$ ). Diese lag bei Patienten mit einer späten klonalen Evolution (>365 d; Gruppe 2) bei 32,56 Monaten ( $n=9$, zensiert: $n=4)$. Dieser Unterschied erreichte kein signifikantes Niveau. Dies ist wahrscheinlich durch die geringe Fallzahl in den jeweiligen Gruppen 
bedingt. Dennoch ist die Tendenz ersichtlich, dass die Patienten mit einer frühen klonalen Evolution auch früher versterben.

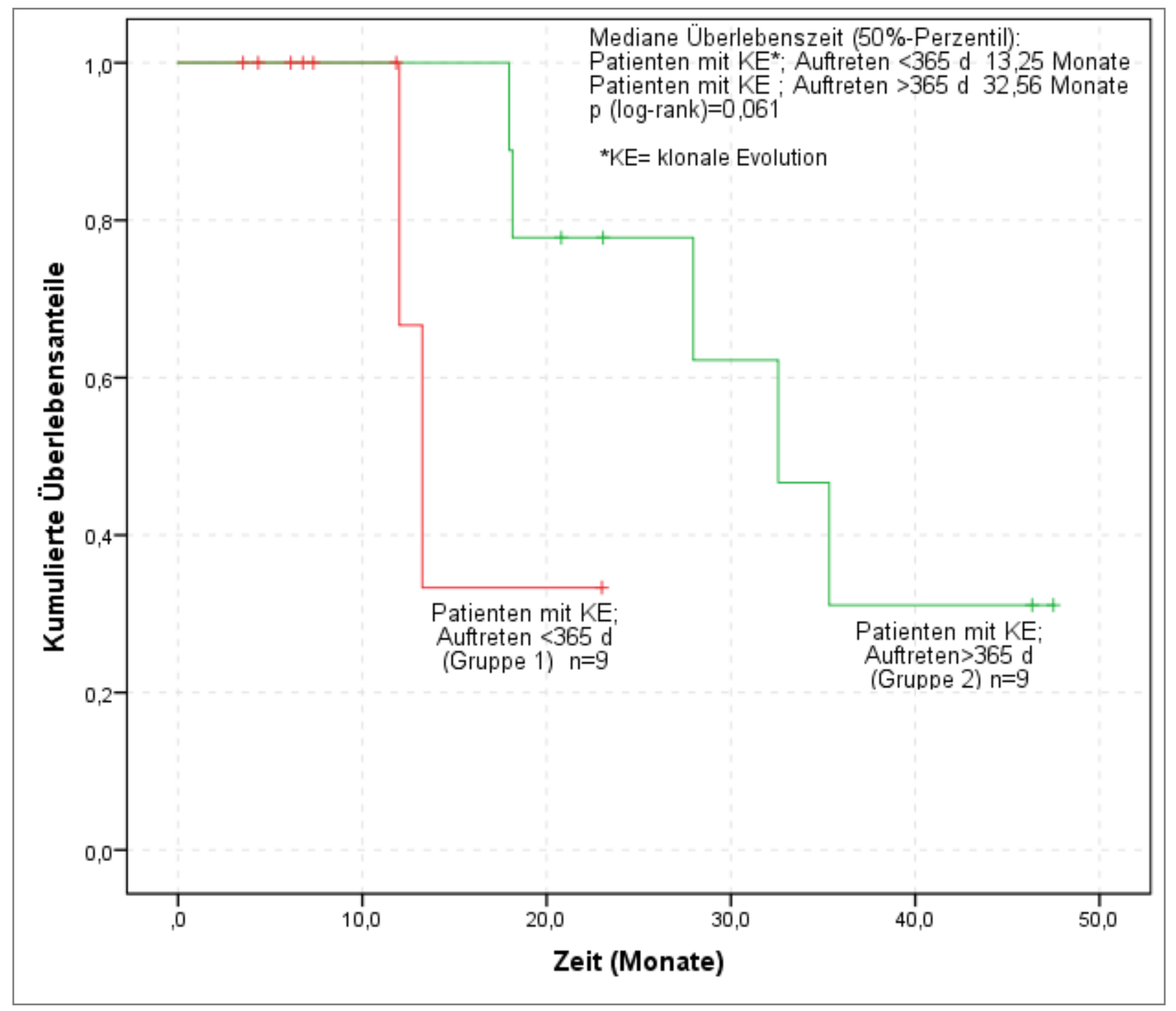

Abbildung 10: Kumulierte Überlebensanteile (Kaplan-Meier) in Abhängigkeit vom Evolutionszeitpunkt: Frühe und späte klonale Evolution

\subsection{Zytogenetische Befunde klonaler Evolution und ihre prognostische Bedeutung}

Für die Darstellung der zytogenetischen Befundkonstellationen wurde die Gesamtgruppe in Patienten mit und ohne klonale Evolution aufgeteilt. Die Gruppe der Patienten mit klonaler Evolution umfasst sowohl die Patienten mit Nachweis einer klonalen Evolution bereits bei Erstdiagnose als auch die Patienten, bei denen erst im Verlauf ihrer Erkrankung eine klonale Evolution nachgewiesen wurde. Allgemeine zytogenetische Befunde (Aberrationen pro Fall, komplexe und nicht-komplexe Aberrationen, Verteilungstyp und Klongröße) werden in Abhängigkeit von klonaler Evolution und deren prognostischer Relevanz im Weiteren dargestellt. 


\subsubsection{Aberrationen pro Fall}

Hier wurde für eine exakte Einschätzung der Komplexität der Chromosomenanomalien die Aberrationen pro Fall nach Heim und Mitelman (1986) erfasst (siehe 2.2.2). Für die Darstellung der prognostischen Bedeutung erfolgte eine Einteilung in fünf Gruppen (Schanz 2003):

\section{Gruppe 1: keine Aberration}

Gruppe 2: 1 Aberration

\section{Gruppe 3: 2 Aberrationen}

Gruppe 4: 3 Aberrationen

\section{Gruppe 5: >3 Aberrationen}

Patienten ohne klonale Evolution zeigten mit 41,4\% die geringste Aberrationsrate. Im Vergleich dazu fand sich bei Patienten mit klonaler Evolution eine hohe Aberrationsrate mit Nachweis von mehr als drei Aberrationen in 43,6\% der Fälle. Dies lässt vermuten, dass zytogenetische Aberrationen bei klonaler Evolution kumulieren. Die Gruppe der Patienten mit klonaler Evolution fasst hier sowohl Patienten, die bereits bei der Erstdiagnose eine klonale Evolution aufwiesen, als auch solche die erst im Verlauf der Erkrankung eine Evolution entwickelten, zusammen. Daher sind Patienten mit einer klonalen Evolution auch in der Gruppe 1 (keine Aberration) und in Gruppe 2 (1 Aberration/Fall) repräsentiert. Diese Patienten entwickelten erst im Krankheitsverlauf eine klonale Evolution. Die Gruppeneinteilung ist auf den Ausgangsbefund bezogen (Tabelle 10).

\section{Tabelle 10: Aberrationen pro Fall in Abhängigkeit von klonaler Evolution}

\begin{tabular}{lccccc}
\hline $\begin{array}{l}\text { Gruppeneinteilung bezogen } \\
\text { auf den Ausgangsbefund }\end{array}$ & \multicolumn{2}{c}{ Patienten ohne KE } & \multicolumn{2}{c}{ Patienten mit KE } & p-Wert \\
\hline Gruppe 1: keine Aberration & $371(n)$ & $\%$ & Anteil $(n)$ & $\%$ & $\left(\mathrm{Chi}^{2}\right)$ \\
Gruppe 2: 1 A/Fall & 177 & 58,6 & 2 & 2,1 & \\
Gruppe 3: 2 A/Fall & 25 & 28,0 & 13 & 13,8 & \\
Gruppe 4: 3 A/Fall & 16 & 3,9 & 29 & 30,9 & $<0,01$ \\
Gruppe 5: $>$ 3 A/Fall & 44 & 7,5 & 9 & 9,6 & \\
\hline
\end{tabular}

\subsubsection{Komplexe Aberrationen}

Berücksichtigt wurden für die Berechnung der Häufigkeiten komplexer Aberrationen ausschließlich aberrante Fälle (NA- und AA-Fälle). Innerhalb der klonalen Evolution zeigte sich eine deutliche Zunahme der Komplexität des Karyotyps. Bei diesen Patienten fand sich in $54,3 \%$ der Fälle ein komplexer Karyotyp. Im Vergleich dazu konnte lediglich in 


\section{Ergebnisse}

21,6\% Fälle bei Patienten ohne klonale Evolution ein komplexer Karyotyp nachgewiesen werden $(p<0,01)$.

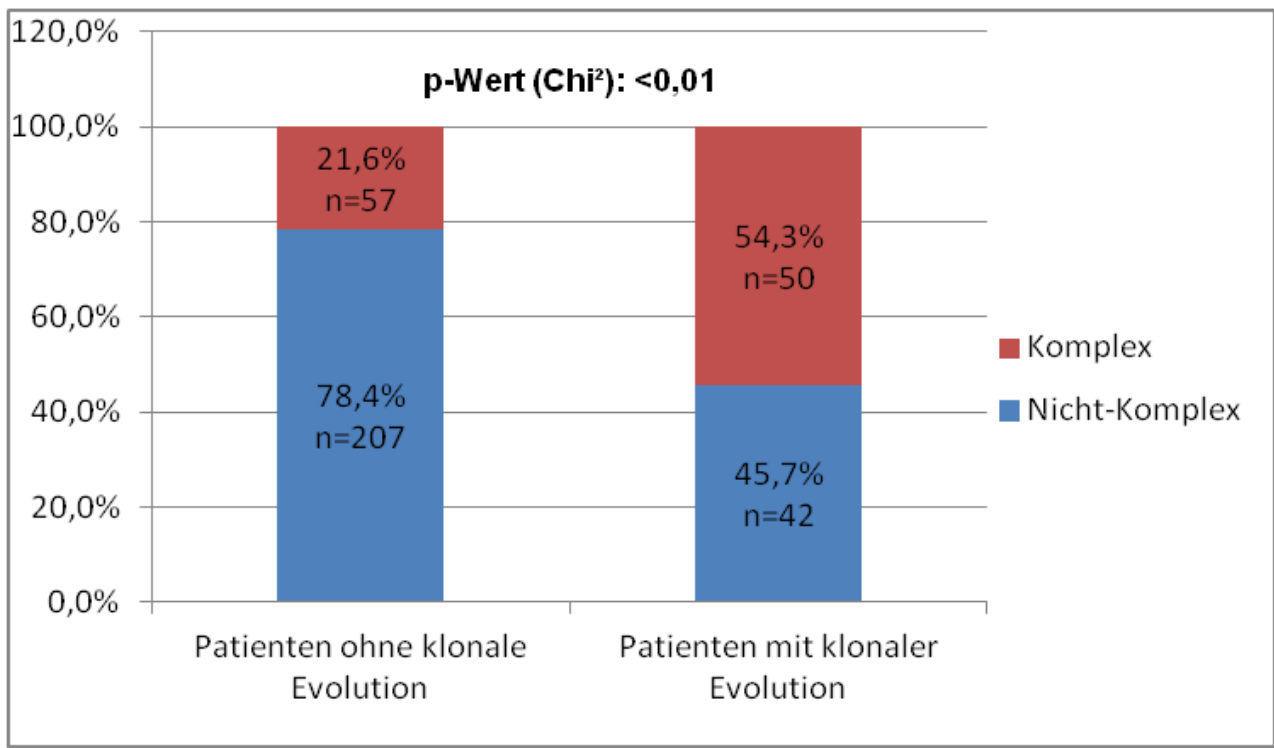

Abbildung 11: Häufigkeit komplexer Aberrationen in Abhängigkeit vom Auftreten klonaler Evolution

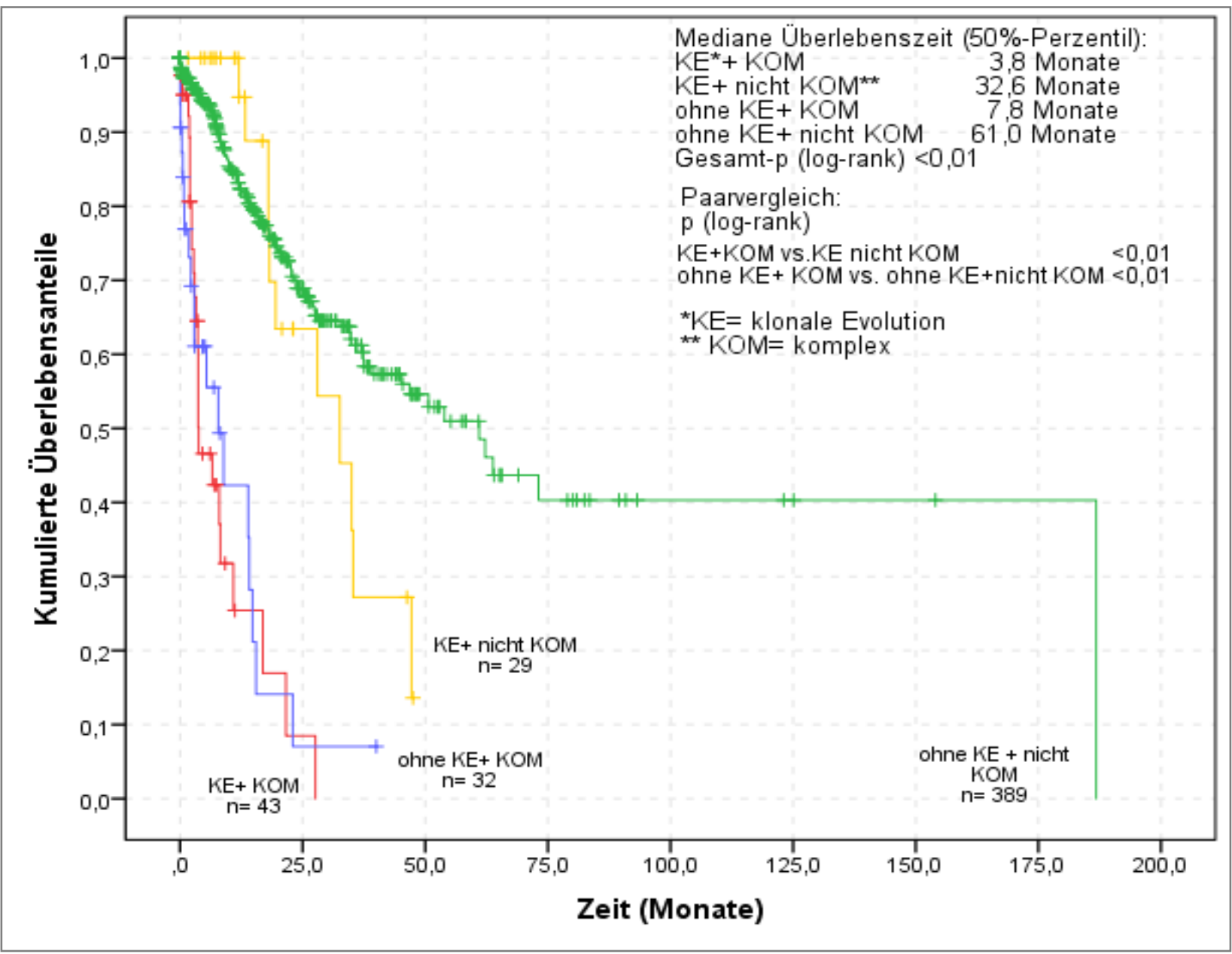

Abbildung 12: Kumulierte Überlebensanteile (Kaplan-Meier) in Abhängigkeit vom Auftreten komplexer Aberrationen mit oder ohne zusätzliche klonale Evolution 
Bei Patienten ohne klonale Evolution fanden sich signifikante Unterschiede im Überleben bei Auftreten eines komplexen Karyotyps (7,8 vs. 61,0 Monate, $p<0,01$ ). Dies konnte auch bei Patienten mit klonaler Evolution festgestellt werden. Die mediane Überlebenszeit verkürzte sich bei Auftreten eines komplexen Karyotyps von 32,6 auf nur 3,8 Monate. Der prognostisch ungünstige Einfluss eines komplexen Karyotyps bleibt innerhalb der klonalen Evolution bestehen (Abbildung 12).

\subsubsection{Verteilungstyp und Klongröße}

Es wurde untersucht, ob die Größe des aberranten Klons eine prognostische Relevanz bei Patienten mit klonaler Evolution hat.

Die klonale Zusammensetzung kann zum einen semiquantitativ durch den Verteilungstyp und zum anderen quantitativ durch die Klongröße dargestellt werden (siehe 2.2.1).

Der Verteilungstyp ist für die semiquantitative Darstellung gut geeignet. Die Klongröße hingegen ist ein $\mathrm{Maß}$ für den genauen prozentualen Anteil aberranter Metaphasen (Schanz 2003, Haase 2005).

Der AA-Karyotyp (ausschließlich aberrante Zellen) konnte in 40,4\% der Fälle mit klonaler Evolution und in nur $12,4 \%$ der Fälle ohne klonale Evolution nachgewiesen werden. Auch trat der NA-Karyotyp (Vorkommen von normalen und aberranten Metaphasen im Mosaik) bei den Patienten mit klonaler Evolution 2-fach häufiger auf (57,4\% vs. $28,3 \%$ ) (Abbildung 13). 
Ergebnisse

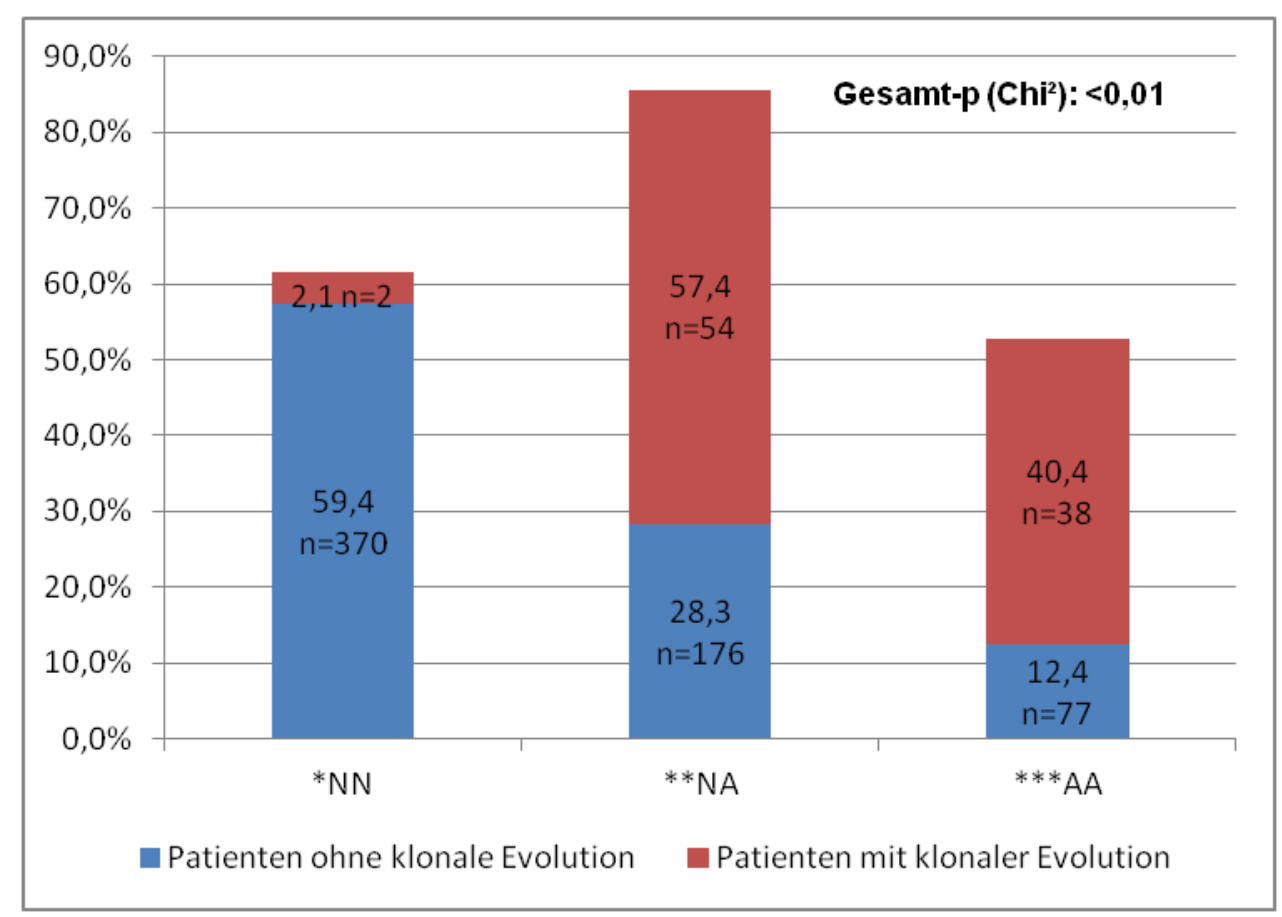

Abbildung 13: Verteilungstyp in Abhängigkeit vom Auftreten klonaler Evolution

${ }^{\star} \mathrm{NN}=$ ausschließlich normale Metaphasen; ${ }^{* *} \mathrm{NA}=$ normale und aberrante Metaphasen im Mosaik; ${ }^{\star \star *} \mathrm{~A} A=$ ausschließlich aberrante Mataphasen

Außerdem fanden sich in der Aufteilung der Klongröße der beiden Patientengruppen deutliche Unterschiede, wie in der Abbildung 14 dargestellt ist.

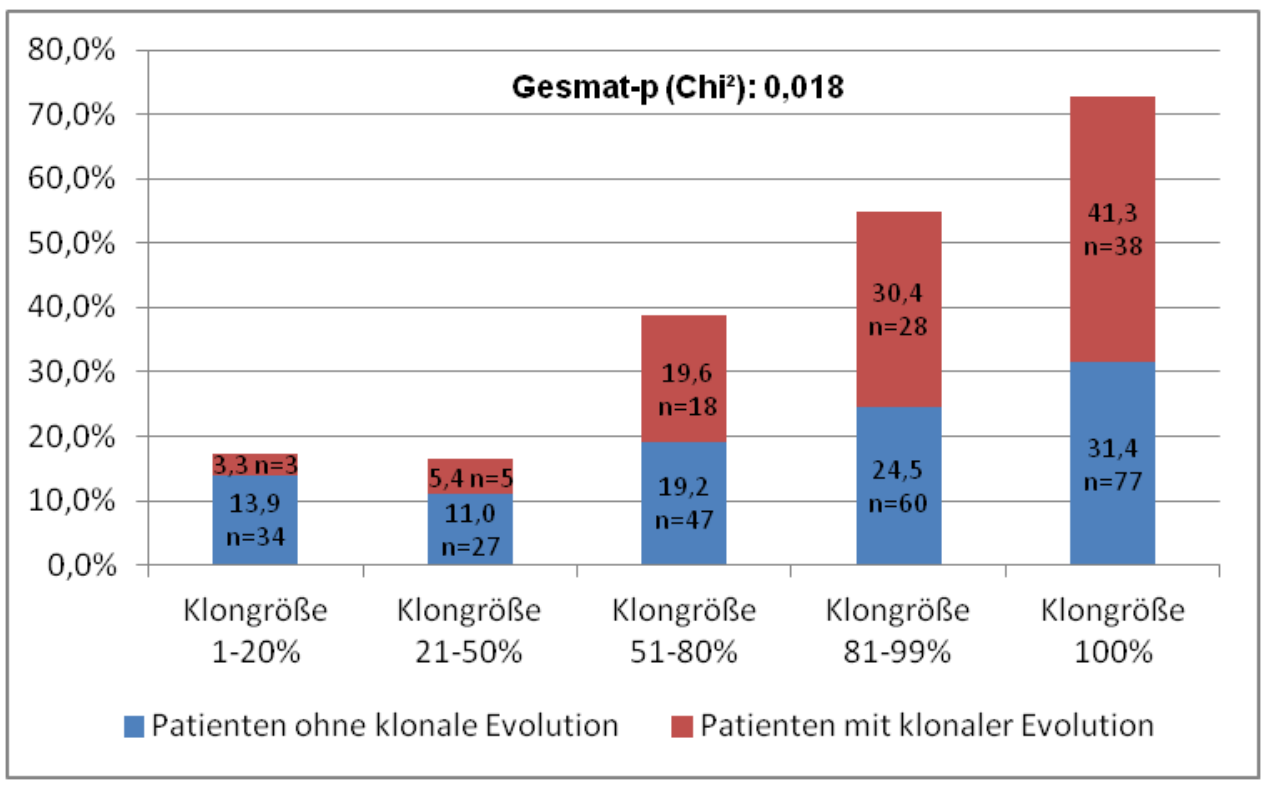

Abbildung 14: Klongröße in Abhängigkeit vom Auftreten klonaler Evolution

Die Berechnungen für die Mittelwerte der aberranten Fälle bei zytogenetischer Erstdiagnose ergaben folgende Ergebnisse: der Mittelwert der Klongröße der Patienten ohne Evolution lag bei $79,2 \%$. Für die Patienten mit klonaler Evolution lag der Mittelwert bei 84,8\% (M-W-U-Test, $\mathrm{p}<0,01)$. 
Sowohl die klonale Zusammensetzung als auch das Hinzukommen von zusätzlichen Aberrationen, wie sie bei klonaler Evolution beobachtet werden, haben einen prognostisch ungünstigen Einfluss auf die mediane Überlebenszeit (Abbildung 15).

Der Einfluss des Verteilungstyps hinsichtlich der Prognose innerhalb der Patienten mit klonaler Evolution bleibt bestehen. Betrachtet man ausschließlich die Patienten mit klonaler Evolution zeigten sich signifikante Unterschiede im Überleben bei Zunahme der Klongröße (18,0 Monate vs. 6,6 Monate, p=0,046). Ein weiterer Vergleich der AAKaryotypen beider Gruppen (Patienten mit und ohne klonale Evolution) zeigte ebenfalls eine Verkürzung der medianen Überlebenszeit (19,5 Monate vs. 6,6 Monate). Auch hier erreichte diese Differenz im Paarvergleich ein signifikantes Niveau mit einem p-Wert von 0,022 . Hier zeigt sich erneut, dass bei einer Klongröße von $100 \%$ das Auftreten zusätzlicher Anomalien zu einer Verschlechterung der Prognose führt.

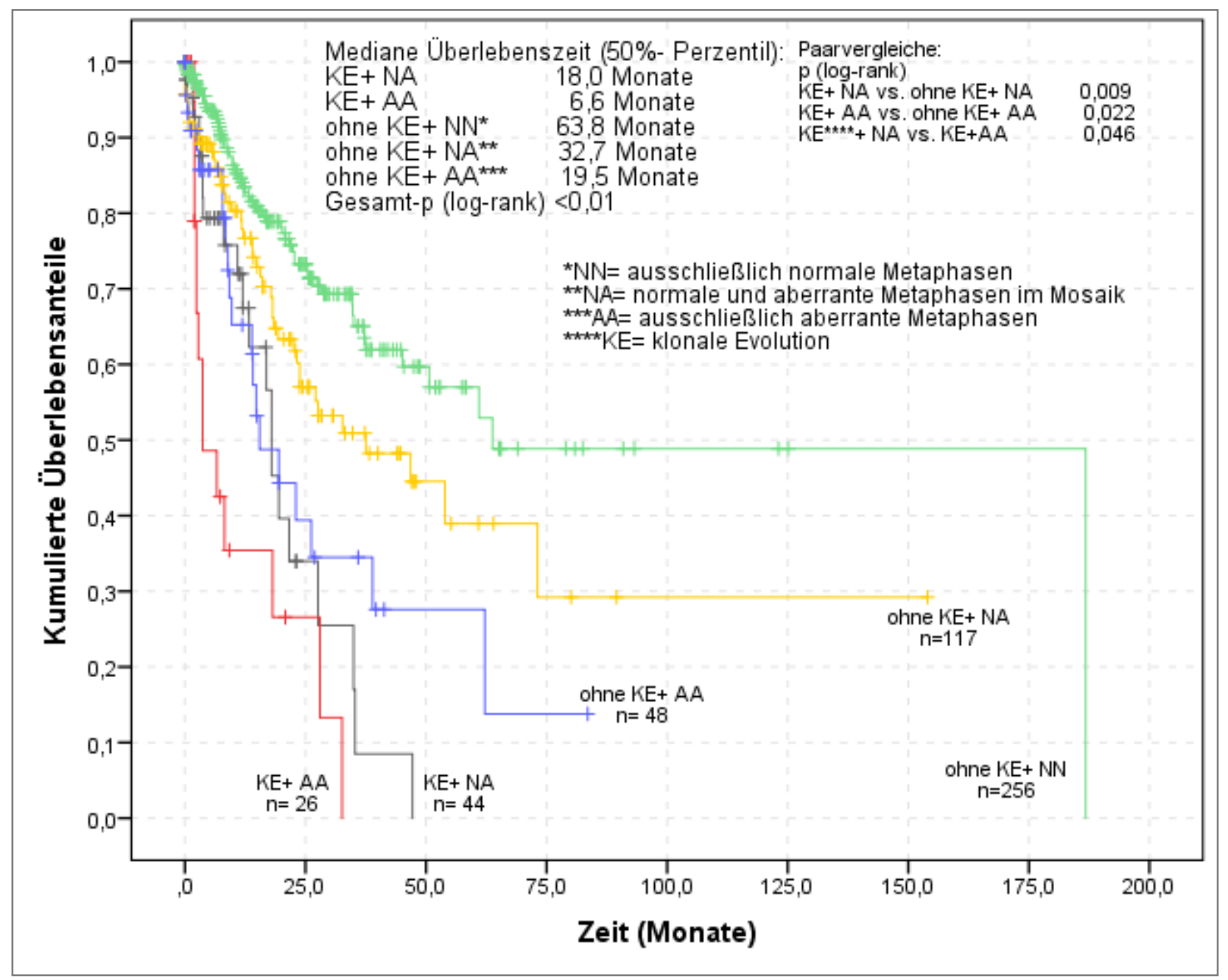

Abbildung 15: Kumulierte Überlebensanteile (Kaplan-Meier) in Abhängigkeit von der klonalen Zusammensetzung und dem Auftreten klonaler Evolution (Cevik et al. 2013) 


\subsection{AML-freies Überleben innerhalb der Gruppe von Patienten mit klonaler Evolu- tion}

\subsubsection{Gesamtkollektiv}

Die Häufigkeit eines Überganges in eine AML war bei Patienten mit und ohne klonale Evolution hochsignifikant unterschiedlich (Abbildung 16). Das Auftreten einer AML bei klonaler Evolution war mehr als doppelt so häufig wie bei Patienten ohne klonale Evolution. So wurde bei 48,0\% ( $n=24)$ aller untersuchten Patienten mit klonaler Evolution ein Übergang in eine AML nachgewiesen. Im Vergleich hierzu zeigten lediglich 21,4\% ( $n=67)$ aller untersuchten Patienten ohne klonale Evolution einen Übergang in eine AML.

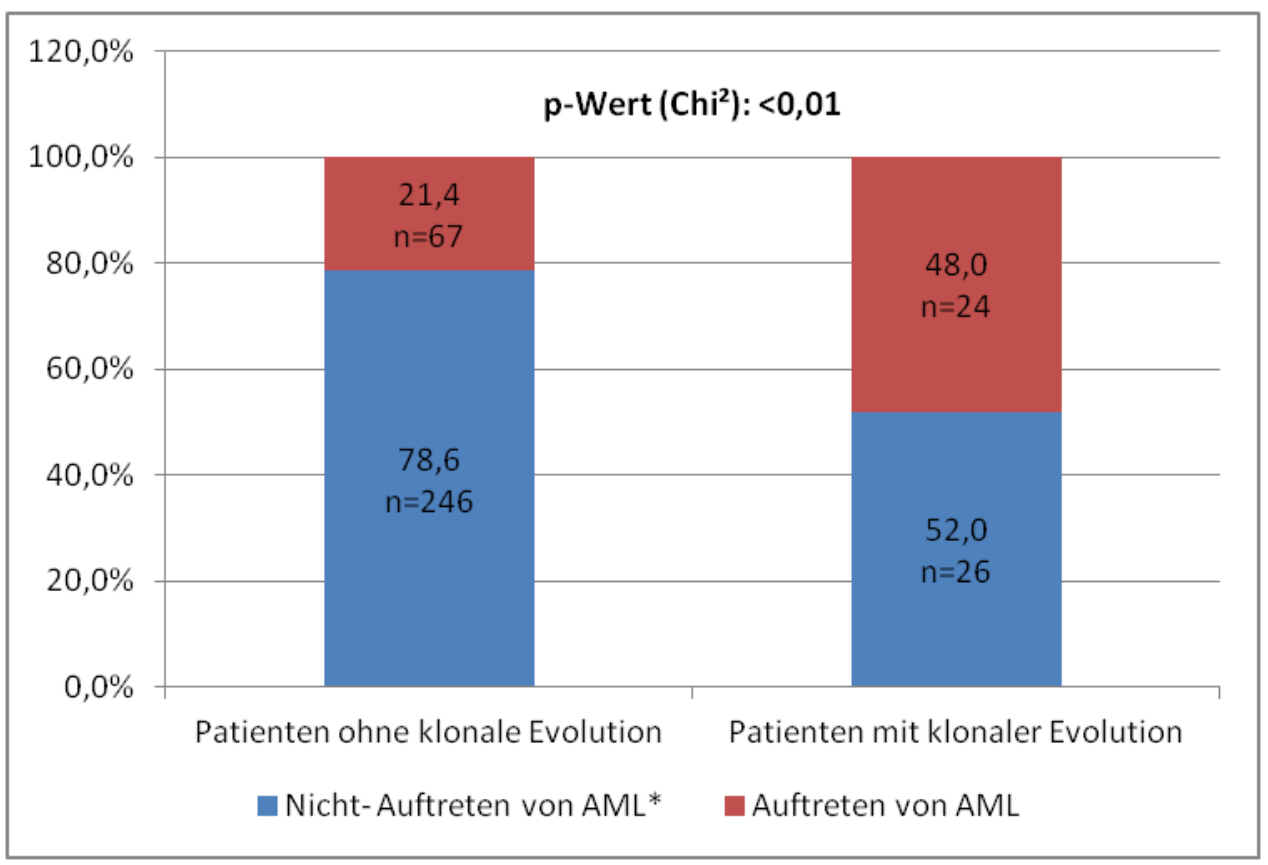

Abbildung 16: Häufigkeit der AML in Abhängigkeit vom Auftreten klonaler Evolution ${ }^{*} \mathrm{AML}=$ akute myeloische Leukämie

Auch hinsichtlich der AML-freien Überlebenszeit konnten hochsignifikante Unterschiede festgestellt werden (Abbildung 17). Bei Patienten ohne klonale Evolution wurden das 50\%und auch das 25\%-Perzentil nicht erreicht. Die Überlebenskurve erreichte ein Plateau. Hieraus lässt sich schlussfolgern, dass nur ein geringer Teil der Patienten einen Übergang in eine AML zeigte. Bei Auftreten einer klonalen Evolution kam es sehr rasch bei allen $\mathrm{Pa}-$ tienten zu einem AML-Übergang. Die AML-freie Überlebenszeit bei Patienten mit klonaler Evolution lag im Median bei 17,5 Monaten. 


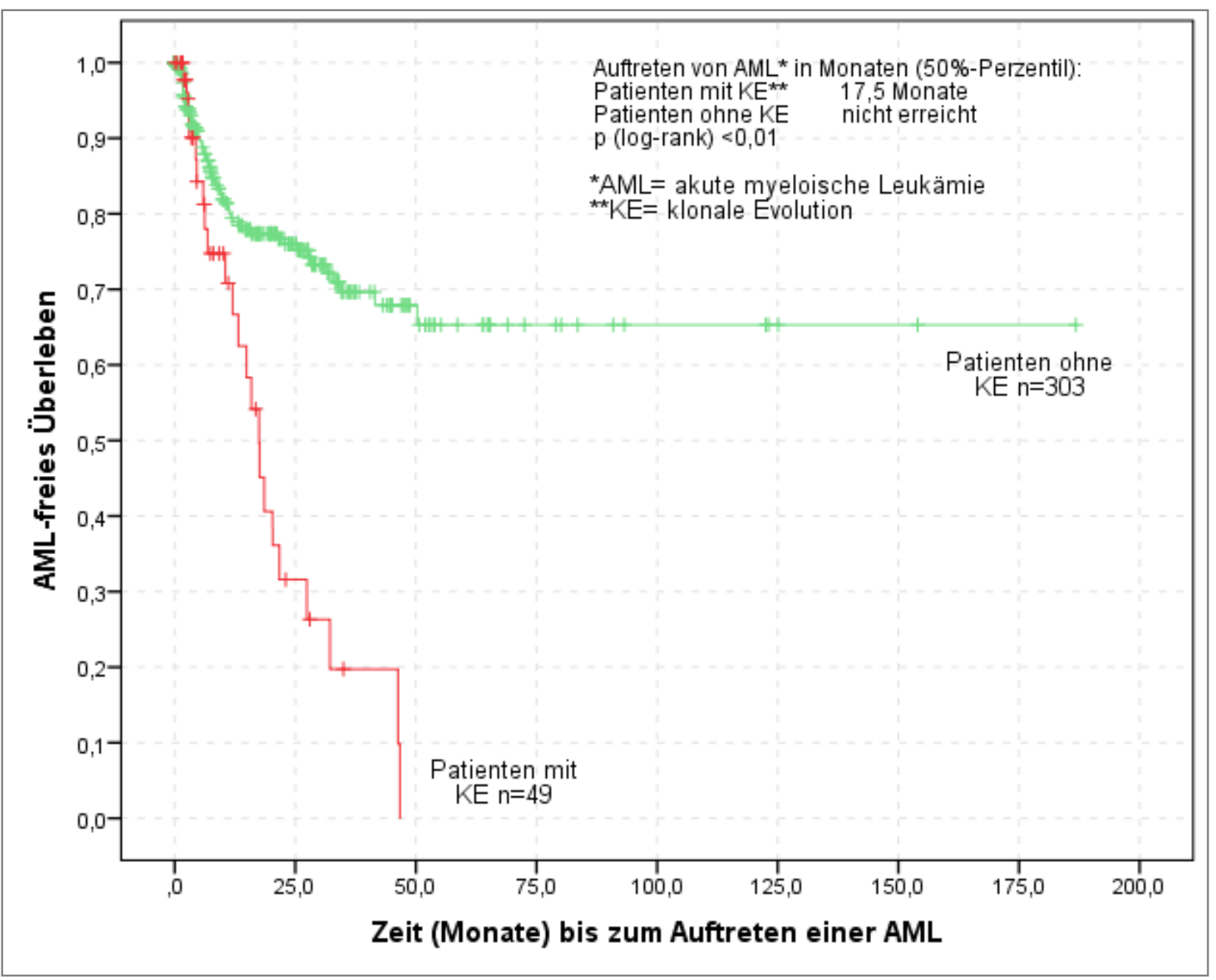

\section{Abbildung 17: AML-freies Überleben der Gesamtgruppe}

\subsubsection{Frühe klonale Evolution}

Die AML-freie Überlebenszeit bei Patienten mit einer frühen klonalen Evolution ( $K E<365 \mathrm{~d}$; Gruppe 1, Vergleich 3.2.4) lag bei 6,8 Monaten (Abbildung 18). Diese verlängerte sich bei Patienten mit einer späten klonalen Evolution (KE >365 d; Gruppe 2) und betrug 20,3 Monate. Somit zeigte sich die Tendenz, dass eine frühe klonale Evolution ebenfalls mit einem frühen Übergang in eine AML assoziiert ist. Vermutlich bedingt durch die geringen Gruppengrößen erreichte dieser Unterschied allerdings keine Signifikanz. 


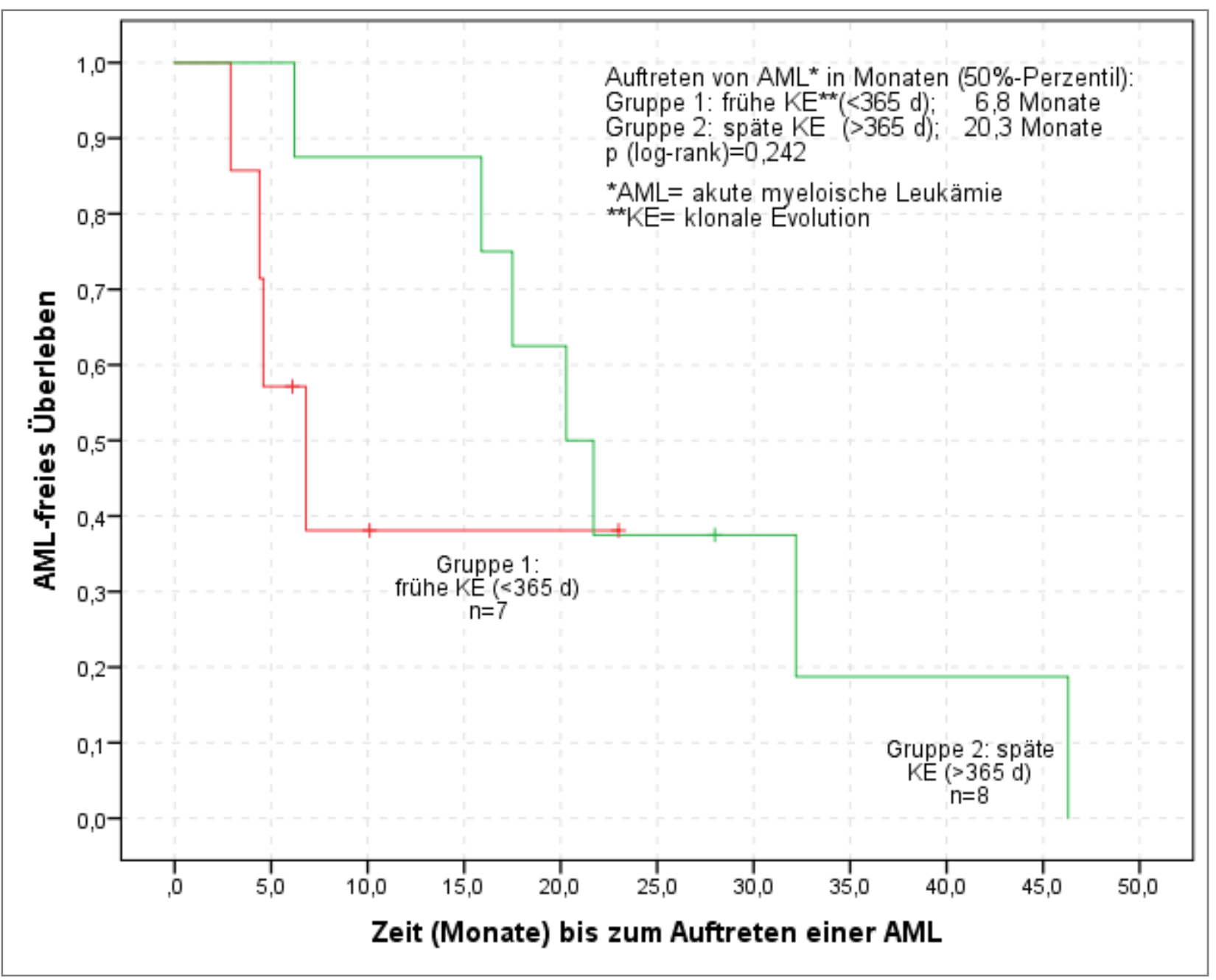

Abbildung 18: AML-freies Überleben in Abhängigkeit vom Evolutionszeitpunkt: Frühe und späte klonale Evolution

\subsubsection{Anomalie-Verteilungstyp}

Untersucht wurde in diesem Abschnitt die AML-freie Überlebenszeit in Abhängigkeit von der klonalen Zusammensetzung. Verglichen wurden Patienten mit und ohne klonale Evolution. In den beiden Gruppen mit einem AA-Karyotyp (Klongröße 100\%; ausschließlich aberrante Metaphasen) zeigte sich kein signifikanter Unterschied (21,7 Monate vs. 32,9 Monate, $p=0,318)$. Im Gegensatz hierzu zeigten sich bei Patienten mit einem NA-Karyotyp (Klongröße 1-99\%; normale und aberrante Metaphasen im Mosaik) signifikante Unterschiede hinsichtlich des Übergangs in eine AML. Patienten ohne klonale Evolution erreichten das 50\%-Perzentil nicht und hatten damit eine bessere Prognose. Bei Auftreten einer klonalen Evolution wurde dagegen eine AML-freie Überlebenszeit im Median von nur 14,9 Monaten berechnet $(p<0,01)$ (Abbildung 19). 


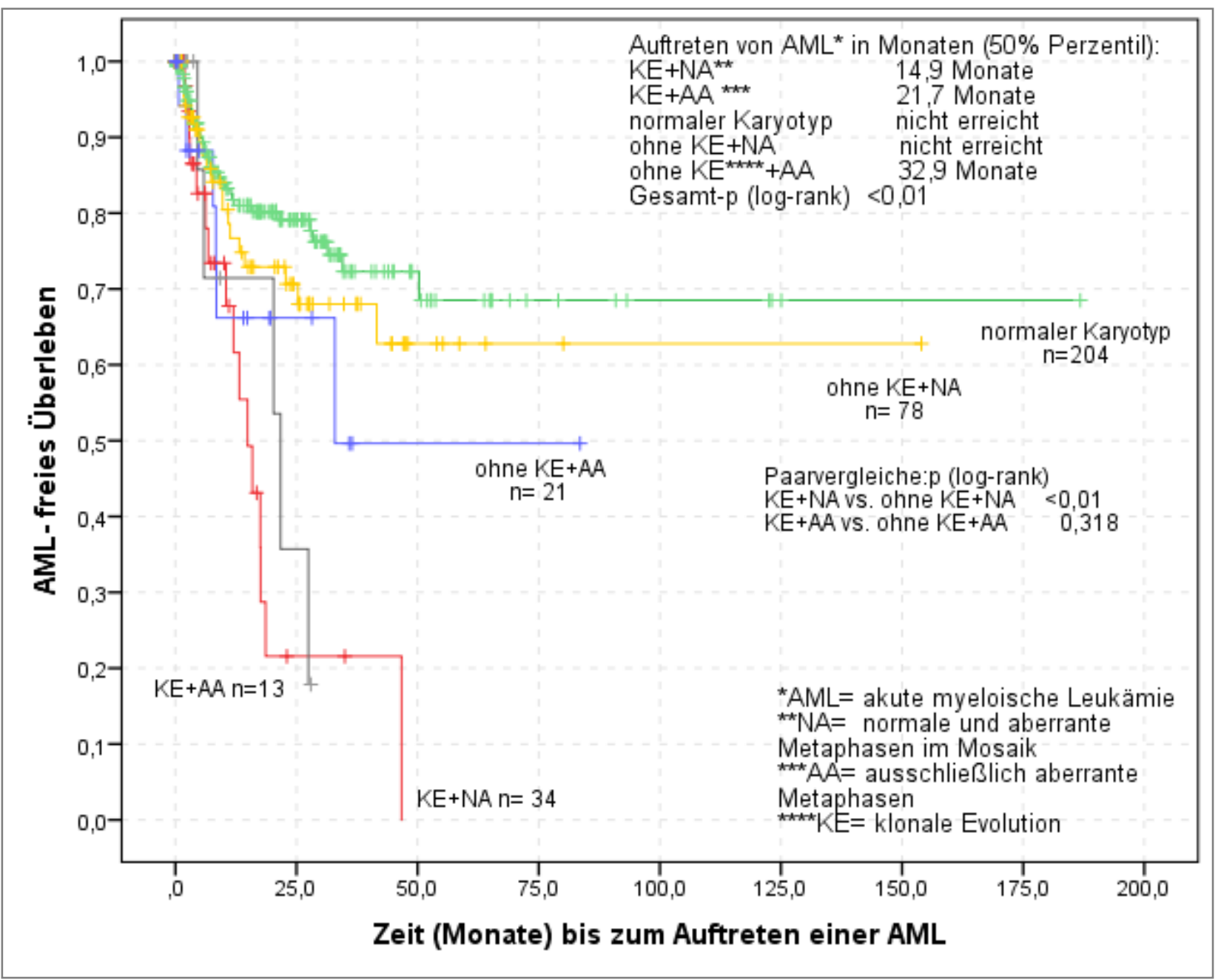

Abbildung 19: AML-freies Überleben in Abhängigkeit von der klonalen Zusammensetzung und dem Auftreten klonaler Evolution

\subsection{Korrelation distinkter Aberrationen mit der klonalen Evolution}

\subsubsection{Häufigkeit spezieller zytogenetischer Aberrationen}

Es wurden sämtliche nachgewiesenen Chromosomenaberrationen für die jeweiligen Chromosomen erfasst und die Häufigkeit innerhalb der Gruppe der Patienten mit klonaler Evolution berechnet. Nachfolgend werden die häufigsten nachgewiesenen Aberrationen in absteigender Anzahl dargestellt: Aberrationen von Chromosom 5 ( $n=51)$, Aberrationen von Chromosom 7 ( $n=35)$ und Aberrationen von Chromosom 8 ( $n=27)$ (Abbildung 20). 


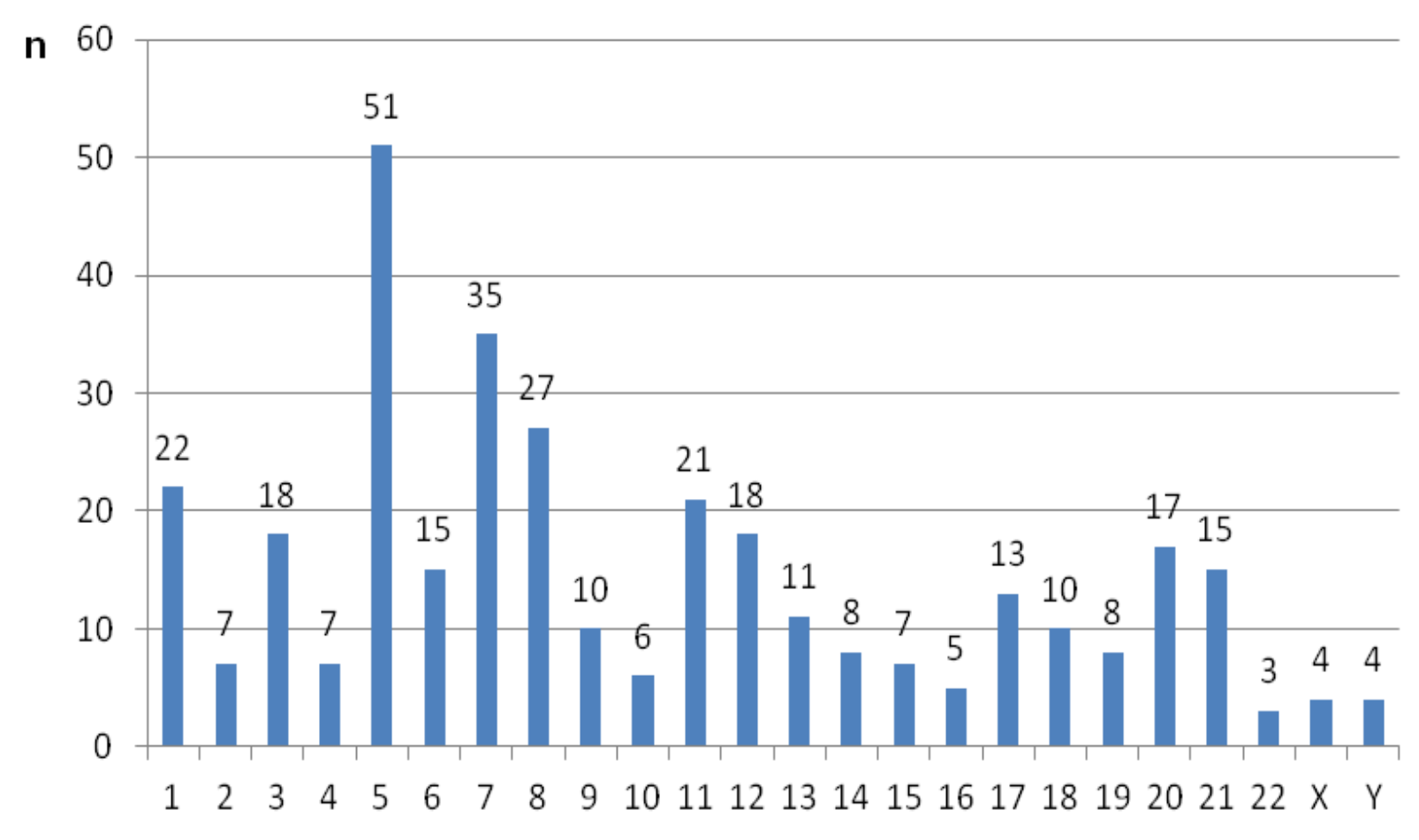

Abbildung 20: Häufigkeit klonaler Aberrationen an einzelnen Chromosomen innerhalb der Gruppe von Patienten mit klonaler Evolution

In den folgenden Abschnitten werden spezielle, mit MDS häufig einhergehende Aberrationen und deren Zusammenhang mit dem Auftreten einer klonalen Evolution, dargestellt. Im Einzelnen wurden folgende, beim MDS häufig auftretende Chromosomenanomalien, näher betrachtet:

- Deletion $5 q$

- Monosomie 7/Deletion 7q

- Trisomie 8

- 17p-Anomalien

- 20q-Anomalien

- Y-Verlust

\subsubsection{Verlust des langen Armes von Chromosom 5 (5q-)}

Die Deletion des langen Armes von Chromosom 5 stellt die häufigste Chromosomenaberration bei Patienten mit MDS dar (Greenberg et al. 1997, Haase et al. 2007, Haase 2008). Diese Anomalie konnte in der vorliegenden Studie bei 109 Patienten (30,6\%) nachgewie- 
sen werden. Der Vergleich der Patienten mit und ohne klonale Evolution zeigte hochsignifikante Unterschiede im Auftreten der 5q-Deletion (Abbildung 21).

Die Patienten mit einer klonalen Evolution wiesen fast doppelt so häufig eine 5q-Deletion auf $(46,7 \%$ vs. $25,0 \%)$. Diese Differenz erreichte ein hochsignifikantes Niveau $(p<0,01)$.

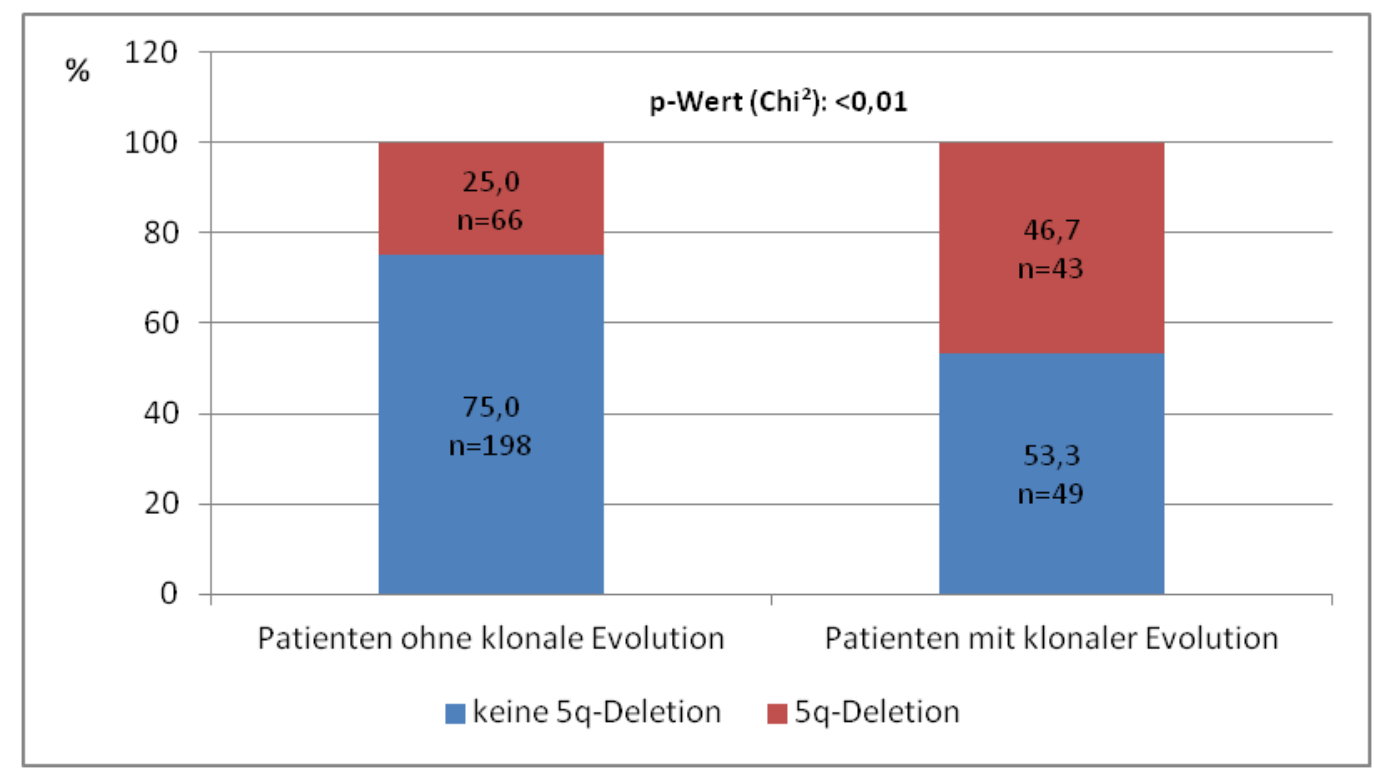

\section{Abbildung 21: Häufigkeit einer 5q-Deletion in Abhängigkeit vom Auftreten klonaler Evolution}

Die Überlebensdaten von 31 Patienten mit klonaler Evolution und Nachweis einer 5qDeletion konnten für die Berechnung der medianen Überlebenszeit herangezogen werden. Zensiert wurden $54,8 \%$ der Fälle $(n=17)$. Patienten ohne klonale Evolution aber mit Nachweis einer 5q-Deletion konnten in 37 Fällen eruiert werden, davon waren 67,6\% der Fälle zensiert.

Patienten mit klonaler Evolution zeigten eine mediane Überlebenszeit von 13,3 Monaten. Die Patienten ohne Evolution erreichten im Vergleich ein Plateau im Überleben. Das 50\%Perzentil konnte nicht errechnet werden. Dieser Unterschied erreichte ein signifikantes Niveau mit einem $p$-Wert von 0,027 . 


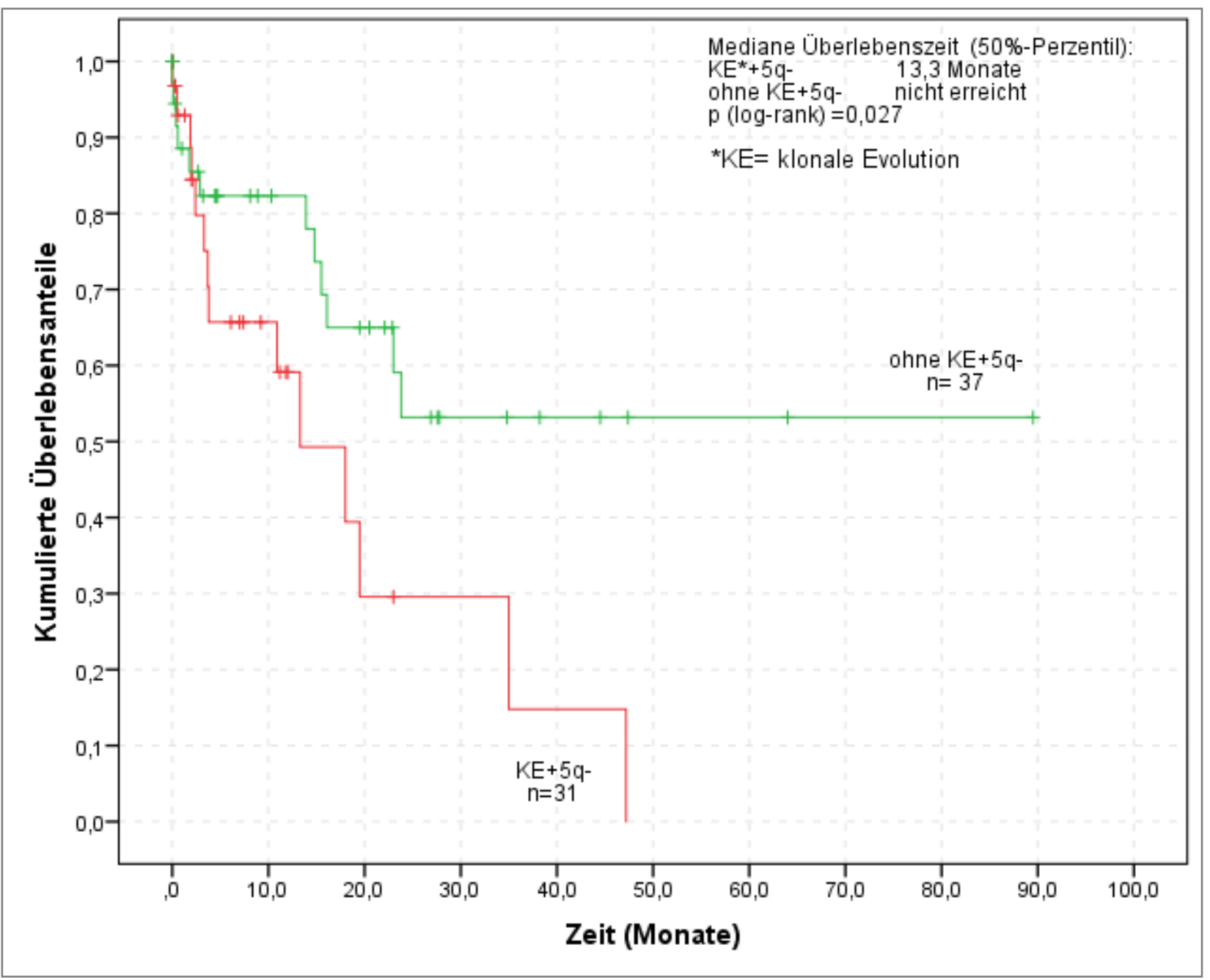

Abbildung 22: Kumulierte Überlebensanteile (Kaplan-Meier) für Patienten mit 5q-Deletion in Abhängigkeit vom Auftreten klonaler Evolution

Inwieweit das Vorkommen einer 5q-Deletion Einfluss auf die Prognose der Patienten mit klonaler Evolution hat, wurde in einer weiteren Berechnung untersucht. Die mediane Überlebenszeit, abhängig von der oben genannten Index-Aberration innerhalb der klonalen Evolution, betrug 13,3 Monate. Ohne Nachweis einer 5q-Deletion lag die mediane Überlebenszeit bei 18,2 Monaten (Abbildung 23). In der Abbildung 22 konnte gezeigt werden, dass sich die ungünstige Prognose der klonalen Evolution auch durchsetzt, wenn ausschließlich Patienten mit 5q-Deletion berücksichtigt werden. Das Auftreten einer 5qDeletion innerhalb der klonalen Evolution zeigte keinen prognostisch relevanten Unterschied im Vergleich zu Patienten mit klonaler Evolution ohne Nachweis einer 5q-Deletion. 


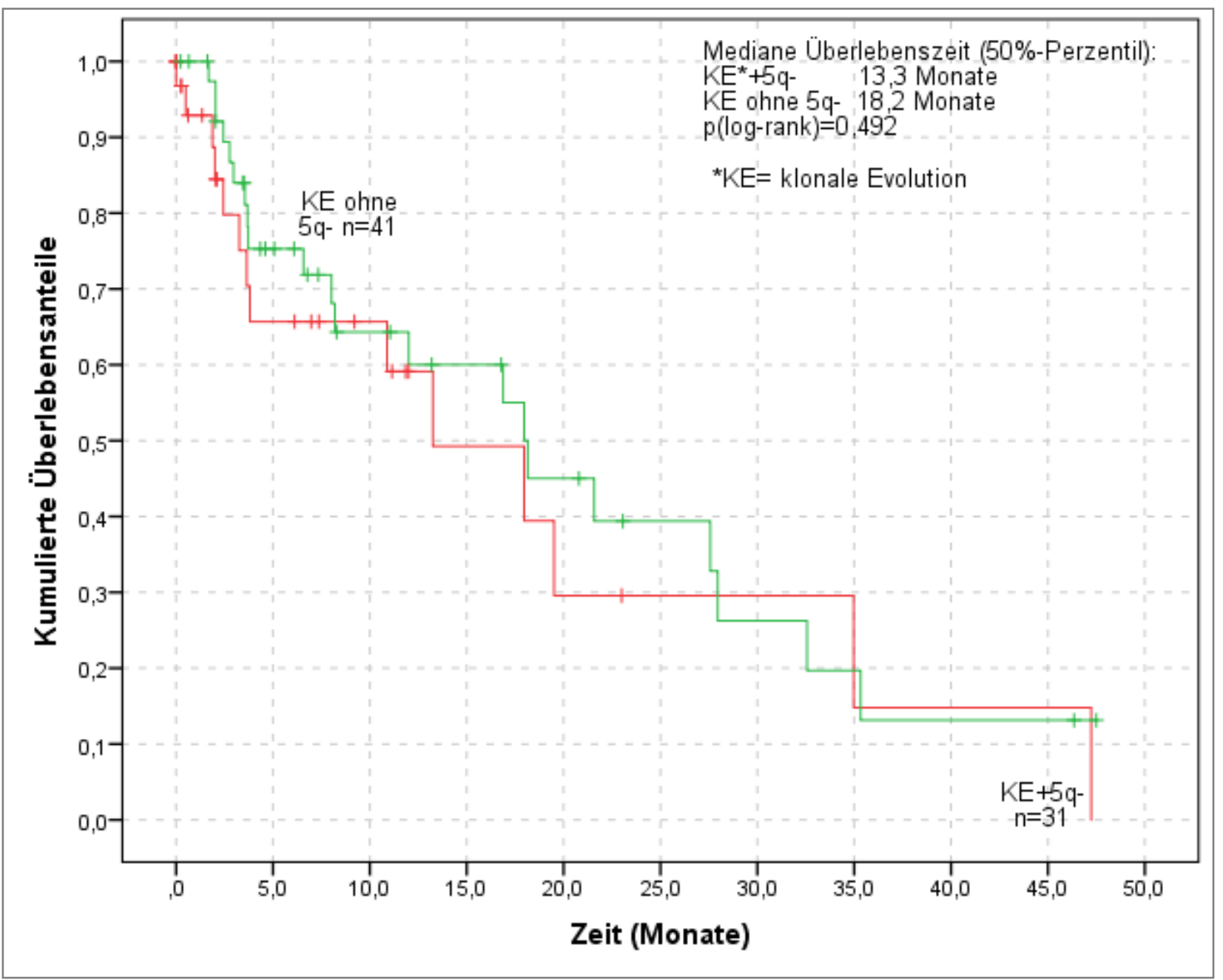

Abbildung 23: Kumulierte Überlebensanteile (Kaplan-Meier) für Patienten mit klonaler Evolution in Abhägigkeit vom Auftreten einer 5q-Deletion

\subsubsection{Monosomie 7/Verlust des langen Armes von Chromosom 7 (7q-)}

Die Monosomie 7 bzw. der Verlust des langen Armes von Chromosom 7 (7q-) konnte bei 88 Patienten (24,7\%) nachgewiesen werden. Hinsichtlich der Häufigkeit des Vorkommens bei Patienten mit und ohne klonale Evolution zeigte sich kein signifikanter Unterschied $(p=0,323)$. $-7 / 7 q-$ fand sich in 29,3\% $(n=27)$ der Fälle mit und in 23,1\% $(n=61)$ der Fälle ohne klonale Evolution (Abbildung 24). 
Ergebnisse

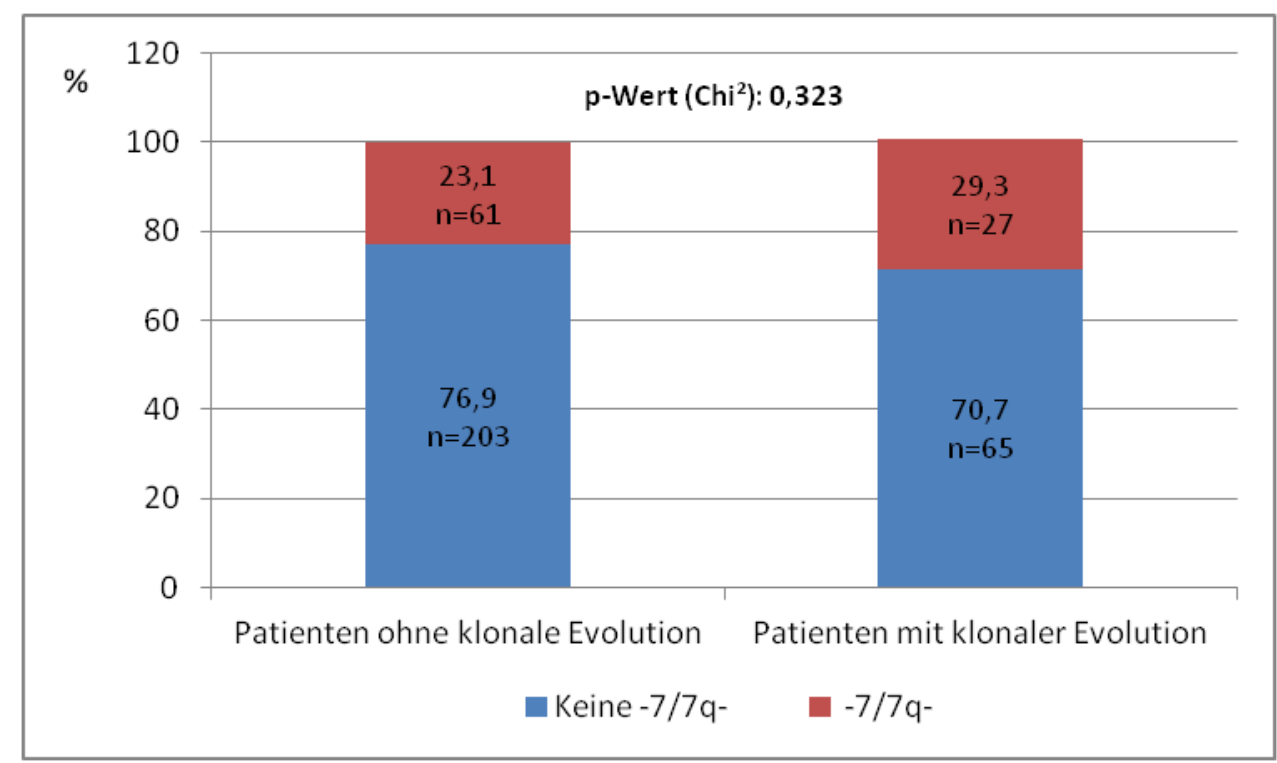

Abbildung 24: Häufigkeit einer totalen oder partiellen Monosomie 7 in Abhängigkeit vom Auftreten klonaler Evolution

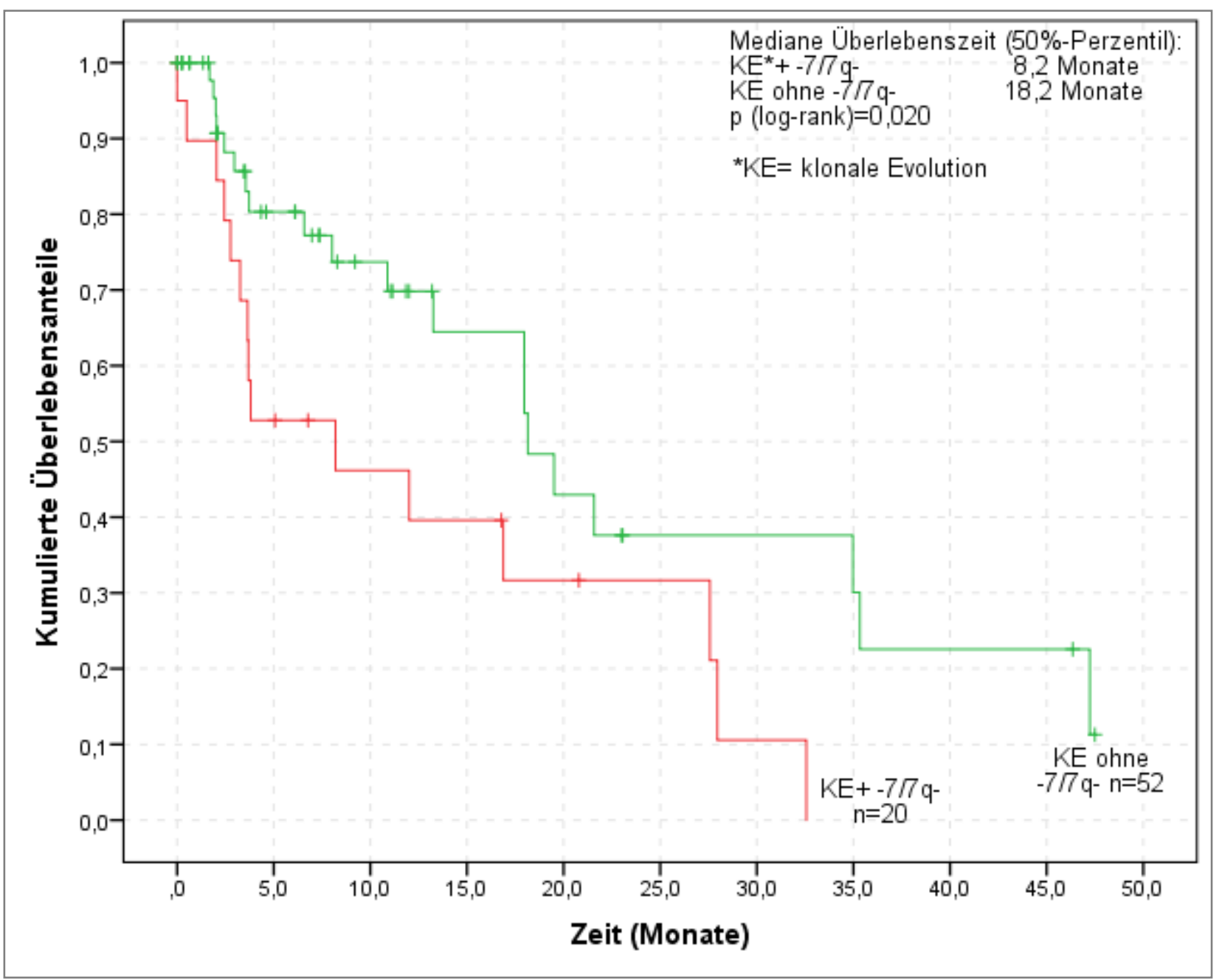

Abbildung 25: Kumulierte Überlebensanteile (Kaplan-Meier) für Patienten mit klonaler Evolution in Abhägigkeit vom Auftreten einer totalen oder partiellen Monosomie 7 
Die prognostische Bedeutung der Monosomie 7/7q-Deletion als Index-Aberration innerhalb der klonalen Evolution erscheint eher ungünstig: So betrug die mediane Überlebenszeit bei Auftreten dieser Aberration 8,2 Monate (Abbildung 25). Patienten mit klonaler Evolution, bei denen diese Index-Aberration nicht nachgewiesen wurde, wiesen eine mediane Überlebenszeit von 18,2 Monaten auf $(p=0,020)$.

\subsubsection{Trisomie 8}

Im Gesamtkollektiv konnte eine Trisomie 8 in 65 der untersuchten aberranten Fälle $(18,2 \%)$ erfasst werden. Dabei wurden in 17,4\% $(n=46)$ der Fälle ohne und in 20,7\% $(n=19)$ der Fälle mit klonaler Evolution eine Trisomie 8 nachgewiesen. Der Vergleich beider Gruppen erreichte kein signifikant unterschiedliches Niveau. Demnach ist die Häufigkeit der Trisomie 8 in beiden Gruppen nahezu identisch (Abbildung 26).

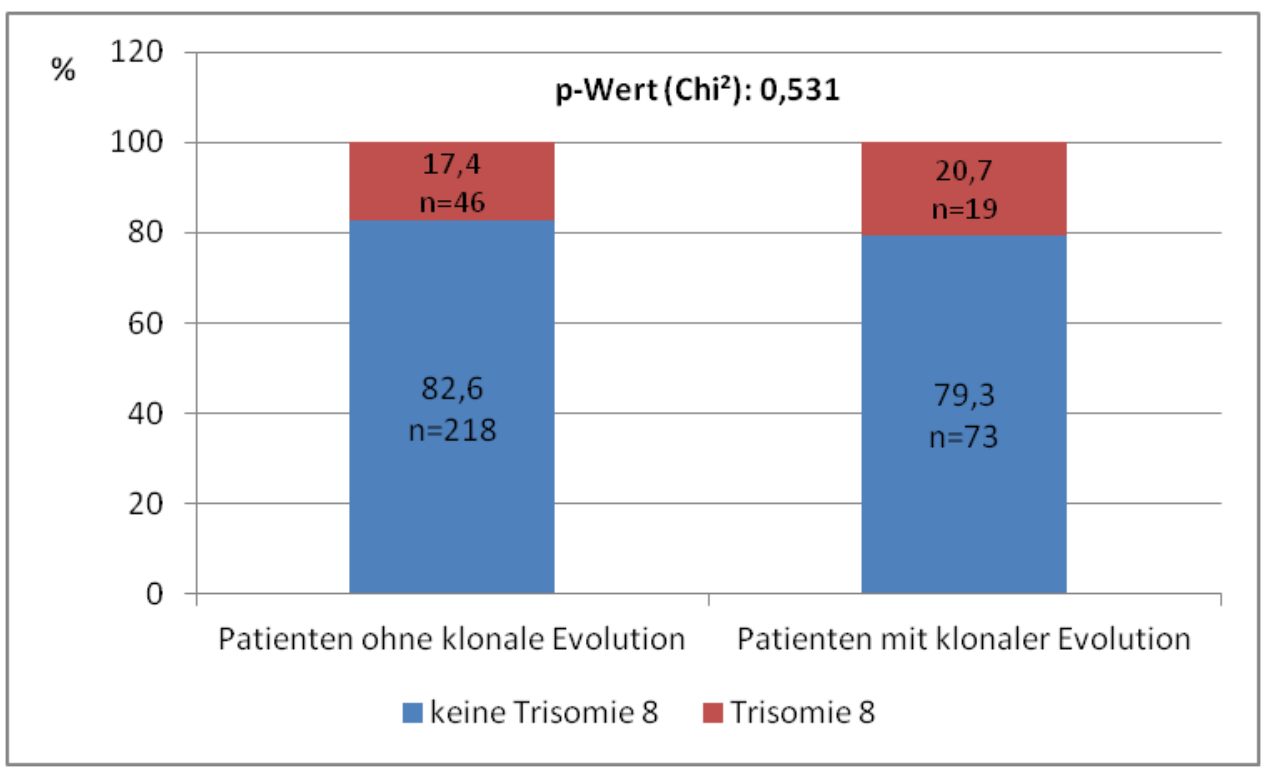

Abbildung 26: Häufigkeit einer Trisomie 8 in Abhängigkeit vom Auftreten klonaler Evolution

Im Gegensatz zur Monosomie 7/7q-Deletion fand sich keine signifikante Verkürzung der Überlebenszeit, wenn die Trisomie 8 als Index-Aberration innerhalb der klonalen Evolution auftrat $(p=0,935)$ (Abbildung 27). 


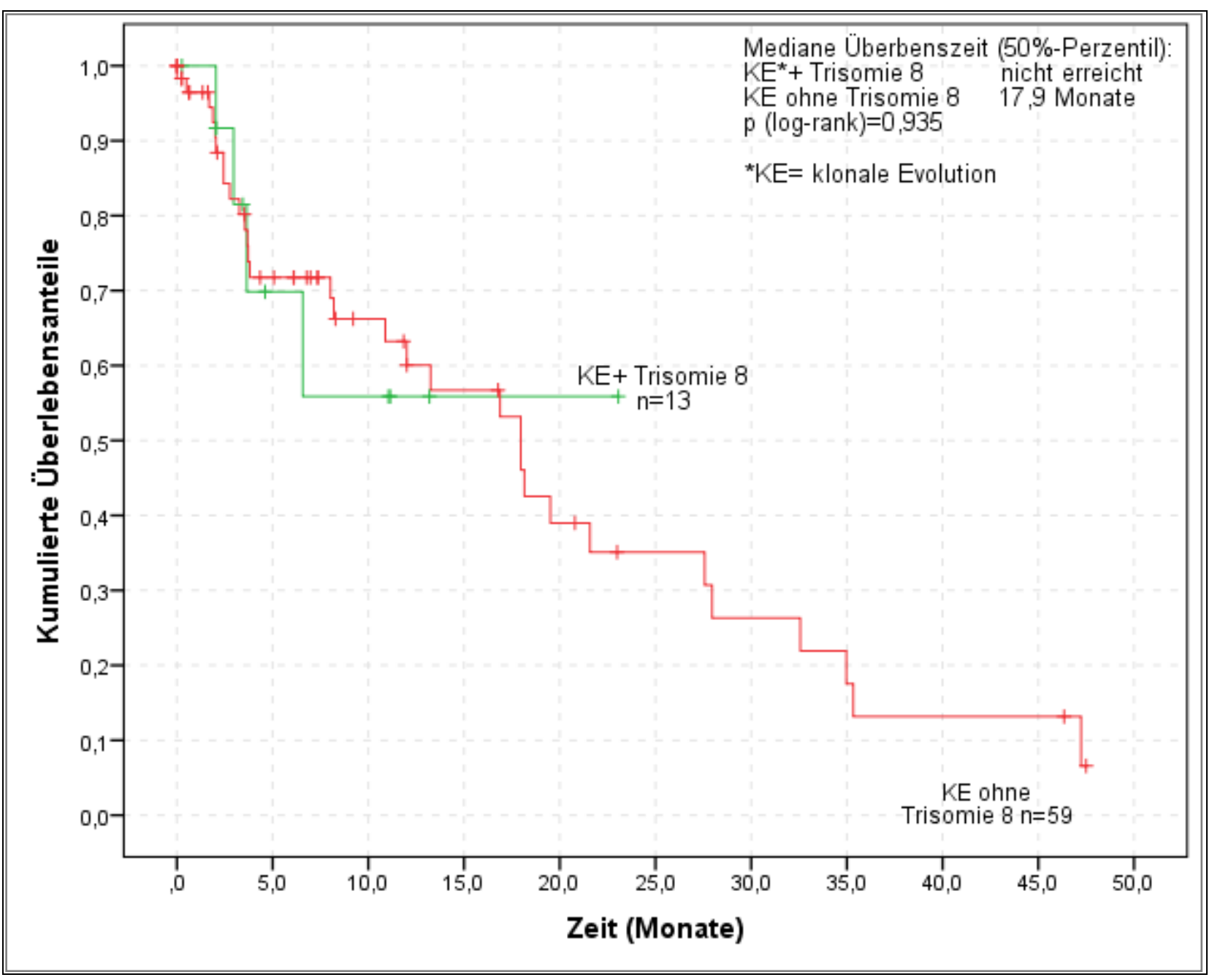

Abbildung 27: Kumulierte Überlebensanteile (Kaplan-Meier) für Patienten mit klonaler Evolution in Abhägigkeit vom Auftreten einer Trisomie 8

\subsubsection{Anomalien des kurzen Armes von Chromosom 17 (17p-Anomalien)}

17p-Anomalien fanden sich im Gesamtkollektiv in acht von 356 aberranten Fällen (Abbildung 28). Bei klonaler Evolution wurde diese Anomalie mit einer Häufigkeit von 5,4\% ( $n=5)$ nachgewiesen, bei Patienten ohne klonale Evolution hingegen in $1,1 \%(n=3)$ der Fälle. Diese Differenz erreichte mit einem $p$-Wert von 0,030 ein signifikantes Niveau.

Hinsichtlich der prognostischen Bedeutung der 17p-Anomalie innerhalb der Patienten mit klonaler Evolution konnte ebenfalls ein signifikanter Unterschied der medianen Überlebenszeit festgestellt werden. Es fand sich eine signifikante Verkürzung der Überlebenszeit, wenn 17p-Anomalien bei Vorliegen einer klonalen Evolution auftraten. Diese betrug 1,8 Monate. Patienten mit einer Karyotypevolution ohne diese Index-Aberration wiesen eine Überlebenszeit von 17,9 Monaten auf ( $p=0,002)$ (Abbildung 29). 
Ergebnisse

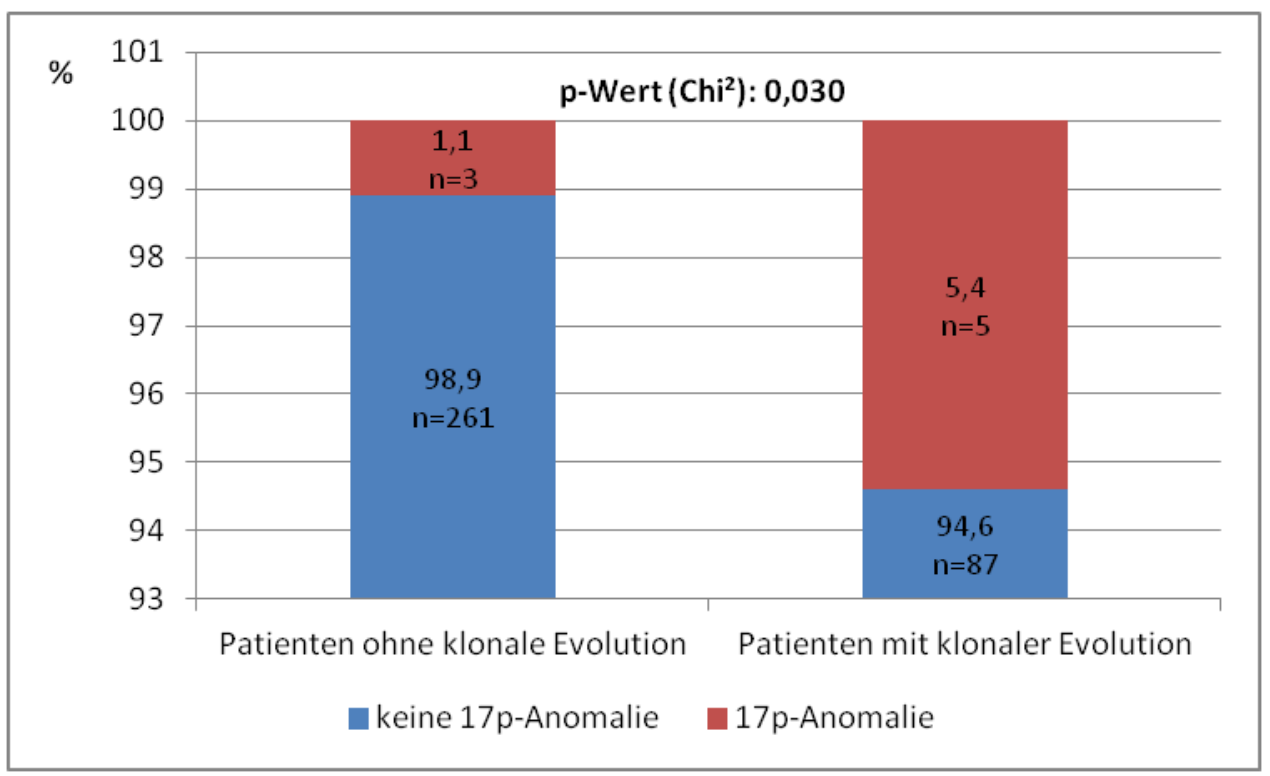

Abbildung 28: Häufigkeit einer 17p-Anomalie in Abhängigkeit vom Auftreten klonaler Evolution

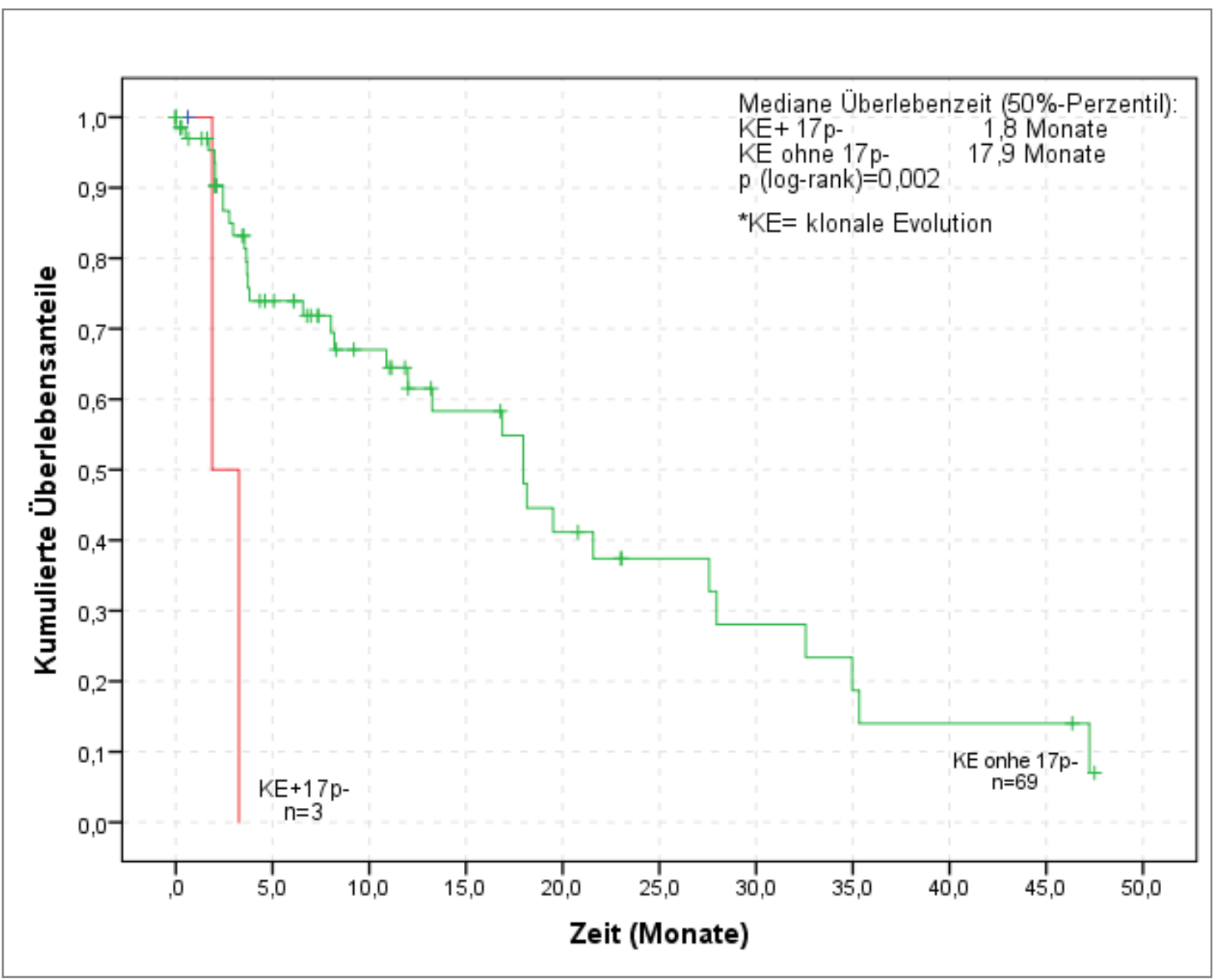

Abbildung 29: Kumulierte Überlebensanteile (Kaplan-Meier) für Patienten mit klonaler Evolution in Abhägigkeit vom Auftreten einer 17p-Anomalie 


\subsubsection{Verlust des langen Armes von Chromosom 20 (20q-)}

In der Häufigkeit des Auftretens der 20q-Deletion innerhalb der beiden Gruppen zeigte sich kein Unterschied. So wurde in 9,8\% der Fälle $(n=9)$ mit und in 5,7\% der Fälle $(n=15)$ ohne klonale Evolution ein Verlust des langen Armes von Chromosom 20 nachgewiesen (Abbildung 30).

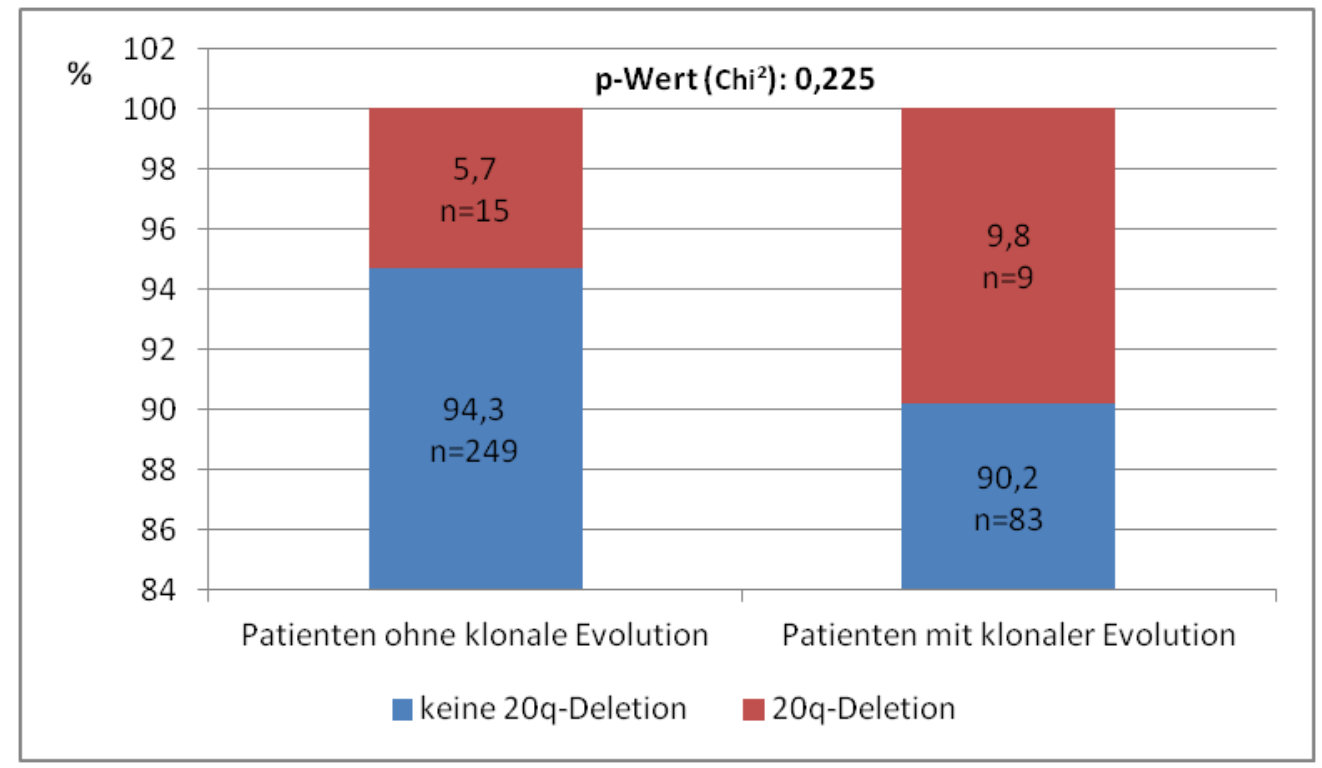

Abbildung 30: Häufigkeit einer 20q-Deletion in Abhängigkeit vom Auftreten klonaler Evolution

Eine signifikante Differenz der medianen Überlebenszeit zeigte sich nicht $(p=0,524)$ (Abbildung 31). So wurde eine mediane Überlebenszeit von 17,9 Monaten bei klonaler Evolution mit Nachweis einer 20q-Deletion berechnet. Patienten ohne Nachweis dieser IndexAberration wiesen eine mediane Überlebenszeit von 16,9 Monaten auf. 
Ergebnisse

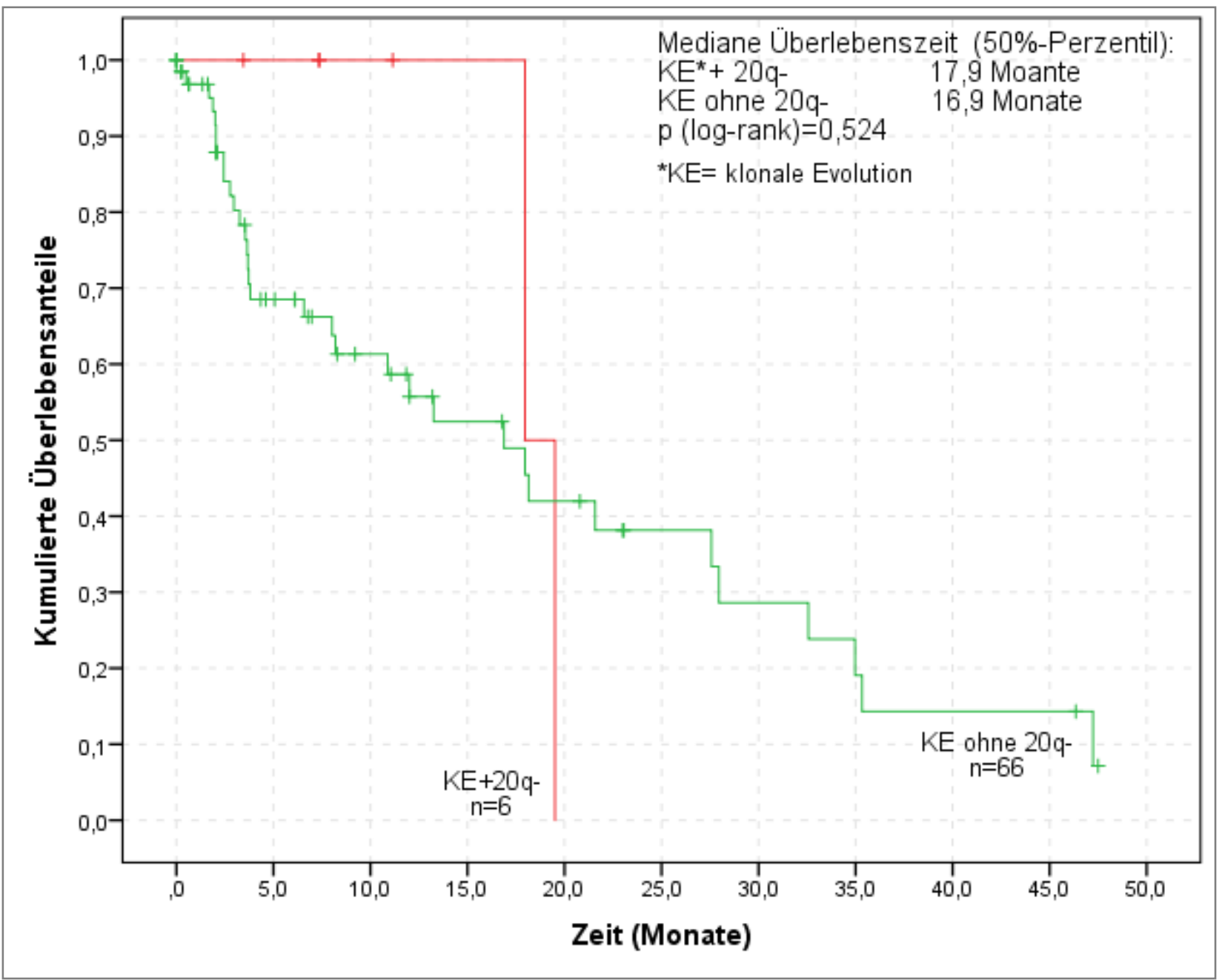

Abbildung 31: Kumulierte Überlebensanteile (Kaplan-Meier) für Patienten mit klonaler Evolution in Abhägigkeit vom Auftreten einer 20q-Deletion

\subsubsection{Verlust des Y-Chromosoms}

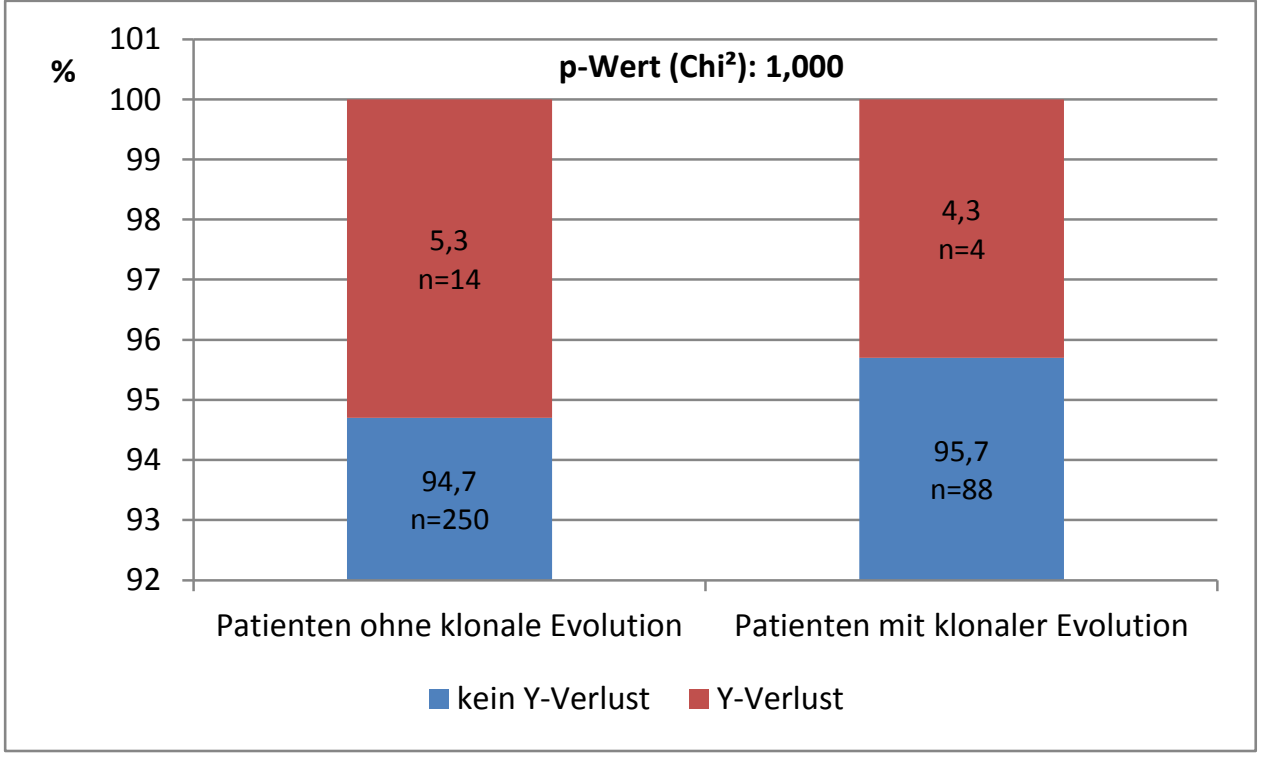

Abbildung 32: Häufigkeit eines Y-Chromosomenverlustes in Abhängigkeit vom Auftreten klonaler Evolution 
Die Häufigkeit von Y-Chromosomenverlusten innerhalb der klonalen Evolution ist mit 4,3\% $(n=4)$ im Vergleich zu 5,3\% ( $n=14)$ bei Patienten ohne Evolution nahezu identisch. Der $p-$ Wert lag bei 1,0 (Abbildung 32).

Signifikante Unterschiede in der Überlebenszeit fanden sich nicht $(p=0,994)$. Innerhalb der klonalen Evolution betrug die mediane Überlebenszeit bei Nachweis eines Y-Verlusts 27,9 Monate. Im Vergleich hierzu lag die Überlebenszeit bei Patienten ohne Y-Verlust bei 17,9 Monaten (Abbildung 33).

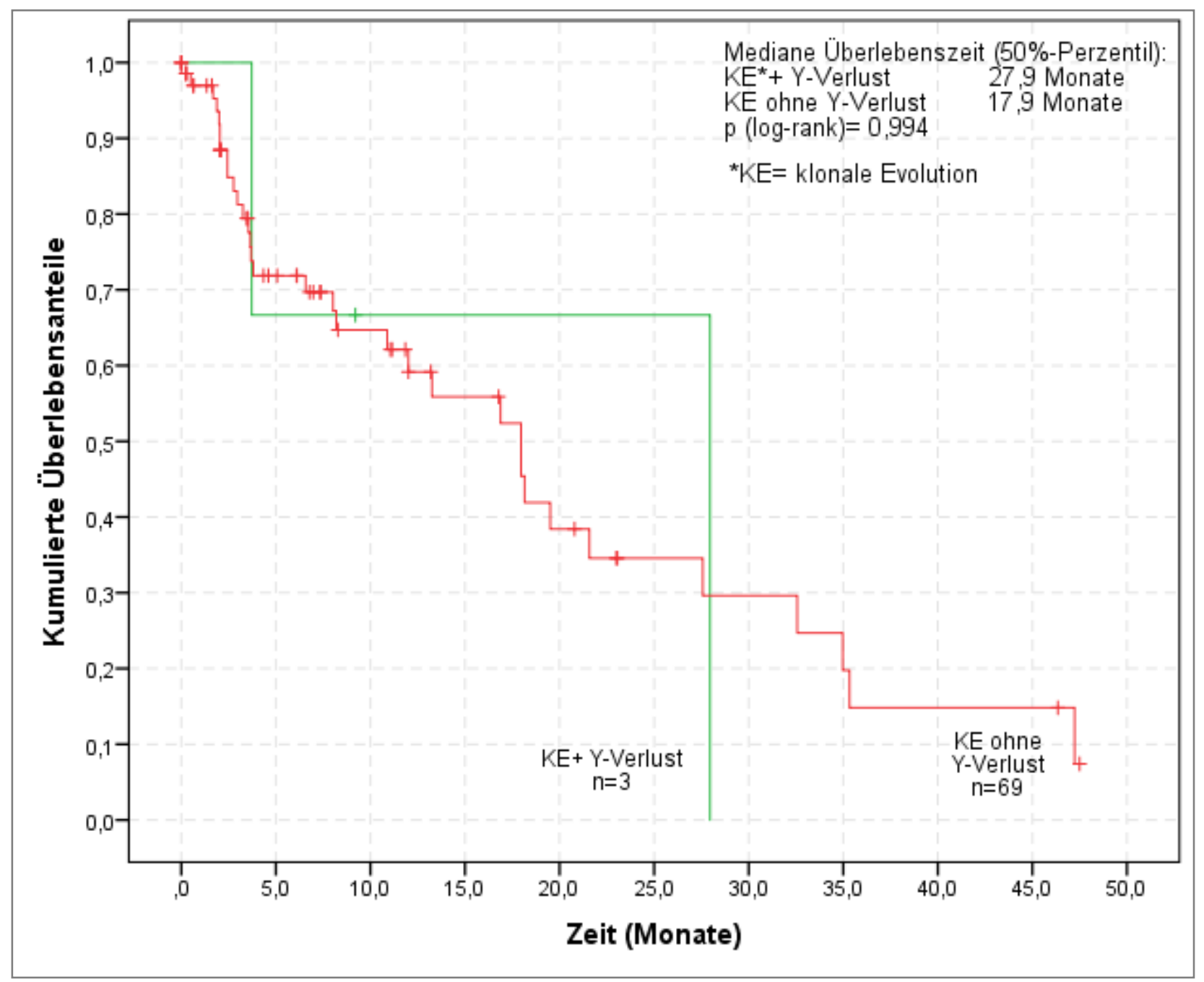

Abbildung 33: Kumulierte Überlebensanteile (Kaplan-Meier) für Patienten mit klonaler Evolution in Abhägigkeit vom Auftreten eines Y-Chromosomenverlustes 


\subsection{Zytogenetische Evolutionsmuster}

\subsubsection{Häufigkeit spezifischer zytogenetischer Evolutionsmuster}

Wie im Abschnitt 2.2.3 erläutert, konnte bei 280 Patienten anhand der zytogenetischen Verlaufsdaten und der Primärdaten eine Einteilung in unterschiedliche Evolutionsmuster erfolgen. 45,0\% der Patienten ( $n=126)$ zeigten einen genetisch stabilen Karyotyp (B), das heißt im Verlauf wurde bei diesen Patienten ein unverändert normaler oder ein unverändert aberranter Karyotyp erfasst. Patienten, die im Verlauf ihrer Erkrankung intermittierend einen normalen Karyotypen aufwiesen, wurden ebenfalls dieser Gruppe subsummiert. Eine „Genetische Progression“ (A) wurde bei 26,1\% $(n=73)$ der Patienten erfasst. Diese Einteilung erfolgte bei Nachweis von Aberrationen bei initial normalem Karyotyp und MosaikKaryotyp sowie bei Auftreten von zusätzlichen Anomalien im Verlauf, aber auch bei einer Zunahme der Klongröße um >20\%. Mit einer Häufigkeit von 19,6\% ( $n=55)$ konnte bereits bei der Erstdiagnose eine klonale Evolution zytogenetisch nachgewiesen werden (D). Bei 21 Patienten, bei denen ebenfalls eine „Primäre Karyotypevolution“ nachgewiesen werden konnte, wurden Verlaufsuntersuchungen erfasst. Diese wurden weiter in die unterschiedlichen klinischen Verläufe eingeteilt. In die Kategorie der "Genetischen Regression“ (C) wurden $6,4 \%$ der Patienten $(n=18)$ eingeteilt. Berücksichtigt werden muss hier, dass 94,4\% dieser Patienten therapiert worden waren und die zytogenetische Regression mit hoher Wahrscheinlichkeit dadurch bedingt gewesen ist. 2,9\% $(n=8)$ der Patienten hatten einen undulierenden klinischen Verlauf (E). Einen Überblick hierzu gibt die Abbildung 34.

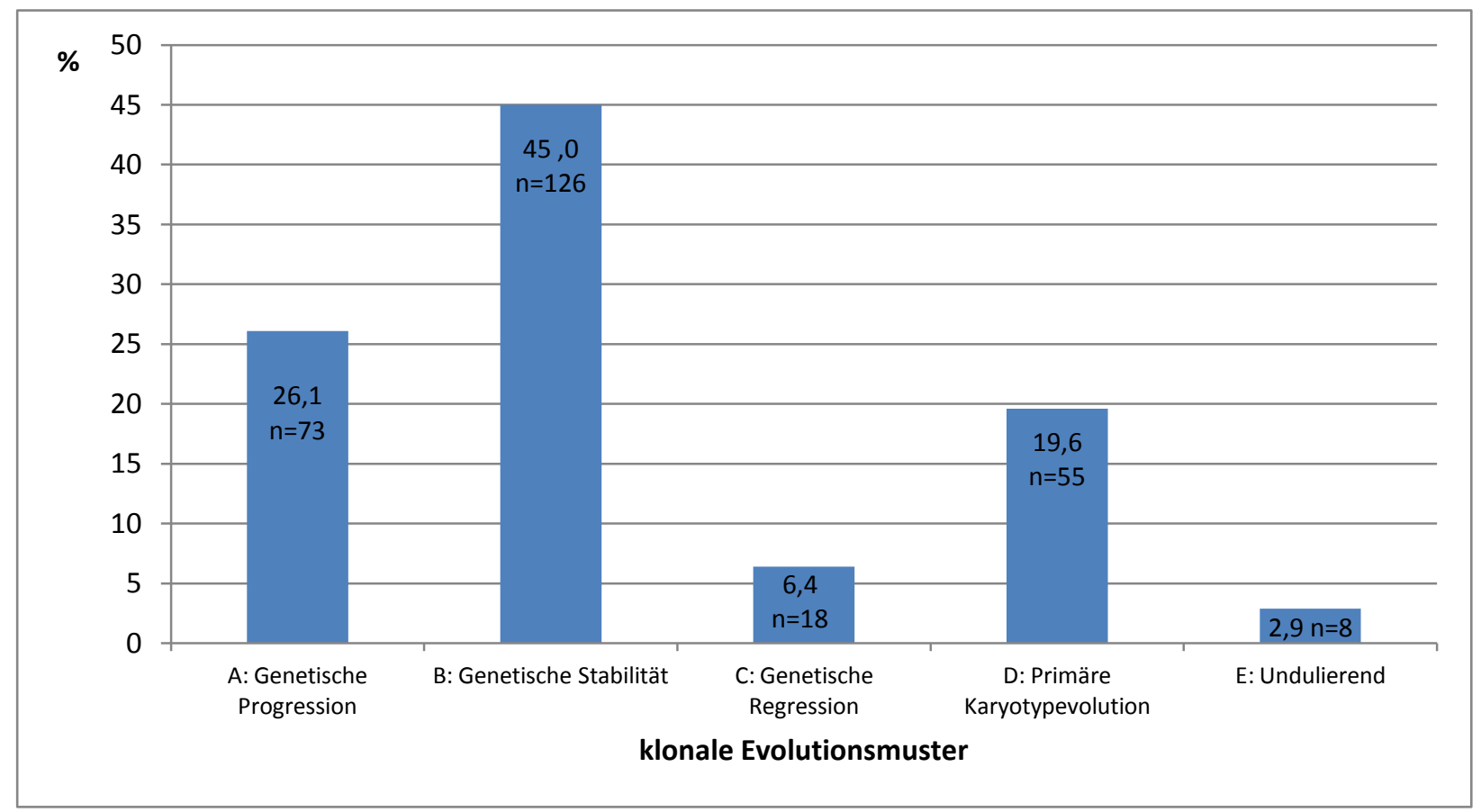




\subsubsection{Häufigkeit komplexer Anomalien innerhalb der klonalen Evolutionsmuster}

Die Untersuchung der komplexen Karyotypen innerhalb der Evolutionsmuster zeigte hochsignifikante Unterschiede $(p<0,01)$. Patienten aus der Gruppe „Primäre Karyotypevolution“ (D) wiesen zu 66,7\% ( $n=36)$ einen komplexen Karyotyp auf. Bei 11,1\% $(n=6)$ der Patienten in der Gruppe „Genetische Progression“ (A) und bei 16,7\% (n=9) der Patienten in der Gruppe „Genetische Stabilität“ (B) fanden sich komplexe Anomalien. Patienten aus der Gruppe „Genetische Regression“ (C) zeigten mit einer Häufigkeit von 5,5\% ( $n=3)$ einen komplexen Karyotyp. Bei Patienten mit einem undulierenden Verlauf fanden sich keine komplexen Aberrationen. Einen Überblick gibt die Tabelle 11.

\section{Tabelle 11: Häufigkeiten komplexer Aberrationen innerhalb klonaler Evolutionsmuster}

\begin{tabular}{lcc}
\hline Evolutionsmuster & Häufigkeiten komplexer Aberrationen & p-Wert $\left(\mathbf{C h i}^{2}\right)$ \\
A: Genetische Progression & $11,1 \%(\mathrm{n}=6)$ & \\
B: Genetische Stabilität & $16,7 \%(\mathrm{n}=9)$ & \\
C: Genetische Regression & $5,5 \%(\mathrm{n}=3)$ & $<0,01$ \\
D: Primäre Karyotypevolution & $66,7 \%(\mathrm{n}=36)$ & \\
E: Undulierend & keine & \\
\hline
\end{tabular}

\subsubsection{Auftreten distinkter Aberrationen innerhalb der klonalen Evolutionsmuster}

Die häufig auftretenden Aberrationen bei MDS-Patienten wurden innerhalb der Evolutionsmuster nach ihrer Prävalenz untersucht (Abbildung 35).

Patienten aus der Gruppe „Primäre Karyotypevolution“ (D) zeigten ein gehäuftes Auftreten einer $5 q$-Deletion mit 58,2\% ( $n=32)$ sowie ein gehäuftes Auftreten einer Monosomie 7/7qDeletion mit 29,1\% ( $n=16)$. Die Trisomie 8 war mit 20,0\% $(n=11)$ in dieser Gruppe ebenfalls häufig. Nur in dieser Gruppe konnten 17p-Anomalien nachgewiesen werden (7,3\%, n=4). Mit gleicher Häufigkeit wiesen die Patienten aus der Gruppe „Genetische Progression" (A) eine Monosomie 7/7q-Deletion mit 16,4\% $(n=12)$ und eine 5q-Deletion ebenfalls mit 16,4\% ( $n=12)$ auf. Eine Trisomie 8 wurde bei $11,0 \%(n=8)$ nachgewiesen. Weniger häufig waren der Nachweis der 20q-Deletion (8,2\%, $n=6)$ und der Y-Chromosomenverlust $(2,7 \%, n=2)$. Die Patienten aus der Gruppe „Genetische Stabilität“ (B) hingegen wiesen eine Trisomie 8 mit 7,9\% $(n=10)$ am häufigsten auf. Die übrigen Chromosomenaberrationen zeigten ein ähnlich häufiges Vorkommen. Bei Patienten aus der Gruppe „Genetische Regression“ (C) fand sich am häufigsten eine Monosomie $7 / 7 q$-Deletion $(27,8 \%, n=5)$. Bei einem undulierenden Verlauf (E) fanden sich folgende Chromosomenanomalien nicht: Monosomie 7/7q-Deletion, 20q-Deletion und 17p-Anomalien. Das Vorkommen von 5qDeletion lag dagegen bei $37,5 \%(n=3)$. 


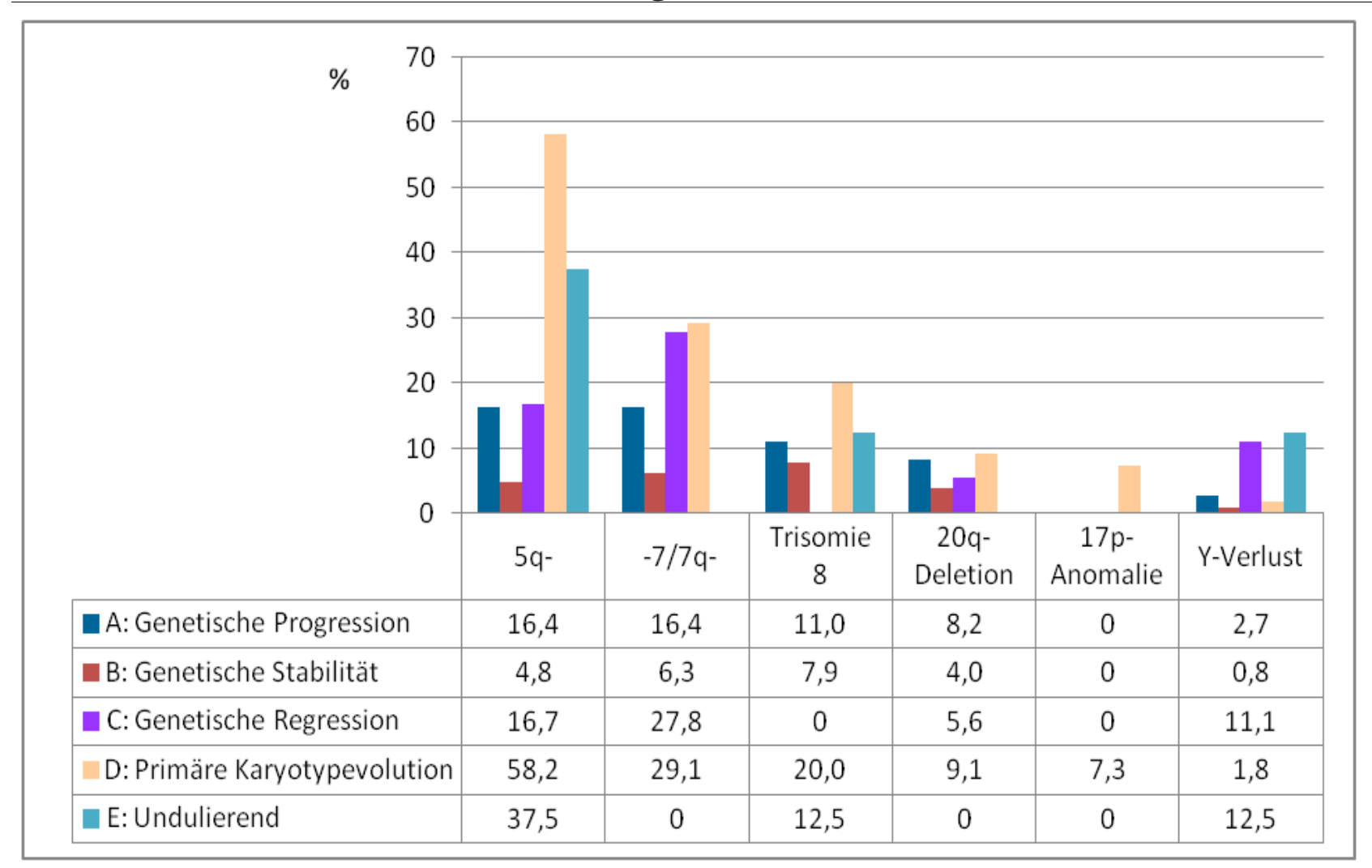

Abbildung 35: Häufigkeit distinkter Aberrationen in Abhängigkeit der klonalen Evolutionsmuster

\subsubsection{Prognostische Bedeutung der klonalen Evolutionsmuster}

Hinsichtlich der medianen Überlebenszeiten zeigten sich innerhalb der zytogenetischen Evolutionsmuster signifikante Unterschiede (Abbildung 36).

Die beste Prognose ergab sich hierbei für Patienten mit einem undulierenden Krankheitsverlauf (E). So konnte hier das 50\%- und auch das 25\%-Perzentil nicht erreicht werden. Ebenfalls wiesen Patienten aus der Gruppe "Genetische Stabilität“ (B) eine gute Prognose auf. Die mediane Überlebenszeit betrug 186,8 Monate. Sowohl bei Patienten aus der Gruppe „Genetische Regression“ (C) als auch aus der Gruppe „Genetische Progression“ (A) zeigten sich deutlich verkürzte Überlebenszeiten. So fiel die mediane Überlebenszeit von 186,8 Monaten auf 37,5 Monate („Genetische Regression“) bzw. 47,2 Monate („Genetische Progression“). Die schlechtere Prognose der Patienten aus der Gruppe „Genetische Regression“ kann möglicherweise Ausdruck eines frühzeitigen Therapiebeginns sein. Möglicherweise wiesen diese Patienten bereits initial eine ungünstige klinische Prognose auf. Auch können Therapienebenwirkungen oder Infektionen während Zytopenien mögliche Erklärungen sein. Die schlechteste Prognose hatten Patienten aus der Gruppe „Primäre Karyotypevolution" (D). Die mediane Überlebenszeit verkürzte sich hier deutlich auf nur 3,6 Monate $(p<0,01)$. 


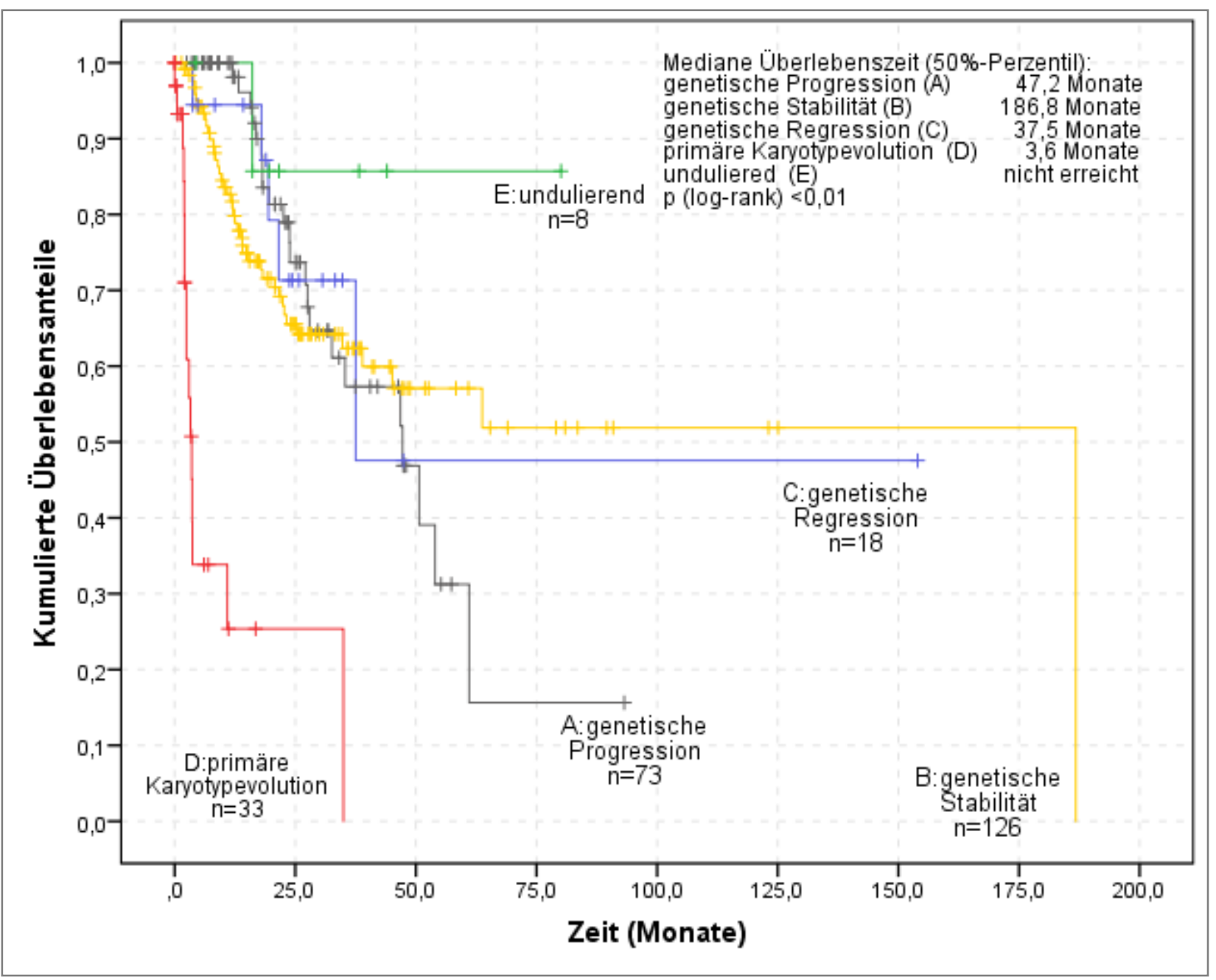

Abbildung 36: Kumulierte Überlebensanteile (Kaplan-Meier) klonaler Evolutionsmuster

\subsubsection{Zytogenetik klonaler Evolution im Krankheitsverlauf}

Hier erfolgte die Untersuchung der Zytogenetik bei Patienten, die im Krankheitsverlauf eine klonale Evolution entwickelten. Verglichen wurde die Zytogenetik bei Erstdiagnose gegenüber der Zytogenetik bei Nachweis einer klonalen Evolution (Tabelle 12).

Als häufigste primäre zytogenetische Aberrationen wurden in absteigender Häufigkeit eine 5q-Deletion ( $n=4)$, Monosomie 7/7q-Deletion $(n=3)$ und 20q-Deletion $(n=2)$ erfasst. Häufige sekundäre Anomalien, die im Verlauf der Erkrankung hinzukamen, waren Monosomie 7/7q-Deletion $(n=3)$ und die Trisomie $8(n=3)$. Der Y-Chromosomenverlust konnte in 2 der 18 Fälle nachgewiesen werden. Weiterhin wurde in 2 der 18 Fälle initial ein normaler Karyotyp erfasst. In einem der Fälle wurde im Verlauf ein komplexer Karyotyp mit Auftreten von Monosomie 6, der(6), Monosomie 17, der(17) sowie Trisomien 8 und 19 nachgewiesen. Im zweiten Fall mit initial normalem Karyotyp zeigte die sequentielle zytogenetische Analyse eine 5q-Deletion und eine Monosomie 21. Die Fallzahl von $18 \mathrm{~Pa}$ - 
tienten die im Verlauf der Erkrankung eine klonale Evolution entwickelten, ist zu klein, um hier eine explizite Aussage über die Anomalieverteilungen zu machen, da die Heterogenität der zytogenetischen Aberrationen dieser Gruppe sehr ausgeprägt ist.

Tabelle 12: Die Zytogenetik der Patienten mit klonaler Evolution im Krankheitsverlauf

\begin{tabular}{|c|c|c|}
\hline $\begin{array}{l}\text { Pat. } \\
\mathrm{Nr}\end{array}$ & ISCN*-Fomel bei Erstdiagnose & $\begin{array}{c}\text { ISCN-Formel bei Nachweis klonaler Evolution im Krank- } \\
\text { heitsverlauf }\end{array}$ \\
\hline 82 & $46, X X, t(1 ; 3)(p 36.1 ; q 21),+21[25]$ & 47,XX, t(1;3)(p36;q21)+21[18]/47, idem, add(17)(p12)[4] \\
\hline 218 & $45, \mathrm{XY},-7[7]$ & 45,XY,-7[?]/45, XY,- 7, t(2;11)(q31;p15)[?]/46, XY [?] \\
\hline 227 & $46, X Y,+i(1)(q), c t b[26], 46, X Y[4]$ & $46, X Y,+i(1)(q 10)[?] / 46, X Y,+i(1)(q 10)$, del 20(q)[?] \\
\hline 247 & $\begin{array}{l}46, \mathrm{XY}, \operatorname{der}(7) \mathrm{t}(1 ; 7)(\mathrm{p} 10 ; \mathrm{q} 10)[39] / \\
46, \mathrm{XY}[5]\end{array}$ & $\begin{array}{c}46, X Y, \operatorname{der}(7) t(1 ; 7)(p 10 ; q 10)[?] / 46, X Y, \operatorname{der}(7) t(1 ; 7)(p 10 ; q 10),+8[ \\
?]\end{array}$ \\
\hline 264 & $45, X,-Y[18]$ & $45, X,-Y[13] / 45, X,-Y, \operatorname{del}(7)(q 22)[5]$ \\
\hline 447 & $46, X X[20]$ & $\begin{array}{c}46, X X[3] / 47, X X,-6,+\operatorname{der}(6) t(1 ; 6)(q 21 p 11.3),+8[4] / 47, X X,- \\
6,+\operatorname{der}(6),+19[2] / 47, X X,+8,-17,+\operatorname{der}(17) t(1 ; 17)(q 21 ; p 13)[11] / 47, \\
X X,-17,-\operatorname{der}(17) t(1 ; ; 17)(q 21 ; p 13),+19[19]\end{array}$ \\
\hline 552 & $46, X X, \operatorname{del}(5)(q 13 q 31)[20] / 46, X X[5]$ & $46, X X \operatorname{del}(5)(q 13 q 31)[22] / 47, X X$, idem,$+4[3]$ \\
\hline 584 & $46, X Y, \operatorname{del}(7)(q 11 q 35)[8] / 46, X Y[6]$ & 46, XY del(7)(q11q35)[18]/45, XY,idem,-22[6] \\
\hline 602 & $47, \mathrm{XX},+8[3] / 46, \mathrm{XX}[22]$ & $46, X X[4] / 47, X X,+8[7] / 48, X X,+8,+21[10]$ \\
\hline 686 & $46, \mathrm{XX}[14]$ & 46, XX, del(5)(q13q31)[7]/45,idem,-21[2]/46, XX[7] \\
\hline 691 & $46, \mathrm{XY}, \operatorname{del}(5)(\mathrm{q} 13 \mathrm{q} 31)[4] / 46, \mathrm{XY}[1]$ & $\begin{array}{c}46, X Y \operatorname{del}(5)(q 13 q 33)[1) / 46, X Y \\
\operatorname{del}(5)(q 13 q 33), t(2 ; 11)(p 21 p 23)[1] / 46, X Y[23]\end{array}$ \\
\hline 693 & $46, \mathrm{XY}, \operatorname{del}(20)(q 12)[1]$ & $\begin{array}{c}46, X Y \operatorname{del}(20)(q 11.2)[3] / 47, X Y,+8, \operatorname{del}(20)(11.2)[3] / 46, X,- \\
Y,+8, \operatorname{del}(20)(q 11.2)[13] / 46, X Y[4]\end{array}$ \\
\hline 749 & $\begin{array}{c}46, X Y[7] / 46, X Y \\
\operatorname{del}(20)(q 11.2, q 13.3)[19]\end{array}$ & 46, XY del(20)(q11.2q13.3)[10]/45, XY, idem,-7[15] \\
\hline 755 & $\begin{array}{l}46, X Y[10] / 46, X Y \\
\operatorname{del}(5)(q 13 q 33)[15]\end{array}$ & $\begin{array}{l}\text { 46, XY [11]/46, XY del(5)(q13q33)[2]/46,XY,idem,del } \\
(16)(q 12.1)[13]\end{array}$ \\
\hline 772 & $45, X Y,-7, \operatorname{del}(3)(q 21 / 23 q 26.2)[25]$ & $45, \mathrm{XY},-7[8] / 45, \mathrm{XY},-7, \mathrm{t}(1 ; 3)(\mathrm{q} 24 \mathrm{q} 26.1)[12] / 46, \mathrm{XY}[2]$ \\
\hline 781 & $\begin{array}{c}\text { 46, } X Y \operatorname{del}(5)(q 13 q 33)[4] / 46 \\
X Y[21]\end{array}$ & $\begin{array}{c}\text { 46, } X Y \text { del(5)(q13q33)[11]/45, } \\
\text { XY,idem,del(8)(q22),der(12)t(12;20)(p12;?),der(13)t(13;18)(p11 } \\
\text {;q?),del(18)(q12),-20[3]/46,XY,[11] }\end{array}$ \\
\hline 800 & $46, \mathrm{XY} \operatorname{der}(3)(q ?)[4] / 46, \mathrm{XY}[2]$ & $\begin{array}{c}\text { 43,X,-Y,-5,+der(5)t(5;16)(q22/31?;?),-7,-16,del(17)(p13),- } \\
\text { 19,+der(19)t(16;19)(?;p10?)[cp5]/44,X,-Y,-1,+der(1p- } \\
\text { t(1;3;6)(p?;?;p25),-15,+der(15)t(15;18)(?;q?),-18,- } \\
\text { 19,+der(19)t(16;19)(?;p10?)[cp6)/46, XY[3] }\end{array}$ \\
\hline 806 & $\begin{array}{c}\text { 46, XX [21]/41- } \\
\text { 45,XX,dic(1;14)(p36.3;p13),- } \\
\text { 5,add(6)(p25),add(9)(q34),t(11;15)( } \\
\text { q12;p13),-15,add(16)(p13),+1- } \\
\text { 2mar [cp10] }\end{array}$ & $\begin{array}{c}46, X X[1] / 45, X X, \operatorname{dic}(1 ; 14)(p 36.3 ; p 13)-10,-14,- \\
16,+2 m a r[13] / 44, X X, \operatorname{dic}(1 ; 14)(p 36.3 ; p 13), \operatorname{add}(7)(p 22),-10,-14,- \\
16,+\operatorname{mar}[2]\end{array}$ \\
\hline
\end{tabular}

${ }^{*} \mathrm{ISCN}=$ International System for Human Cytogenetic Nomenclature 


\subsection{Multivariate Analysen}

Im Folgenden wurde der gleichzeitige Einfluss von mehreren Variablen auf die Überlebenszeit und die AML-freie Überlebenszeit in multivariaten Analysen untersucht. Folgende Einflussfaktoren wurden einbezogen:

- Alter

- Geschlecht

- Klinische Daten (Werte für Hämoglobin, Leukozyten, Thrombozyten und Knochenmark-Blastenanteil)

- Therapie

- Aberrationen pro Fall

- klonale Evolution

- klonale Evolutionsmuster

\subsubsection{Gesamtüberleben in der multivariaten Analyse}

In der multivariaten Analyse zeigte sich, dass die Höhe des Blastenanteils im Knochenmark signifikant mit der Höhe des Sterberisikos assoziiert war. Patienten mit Knochenmark-Blasten von 5\%-10\% und 11\%-20\% hatten im Vergleich zu Patienten mit $<5 \%$ Blasten im Knochenmark ein 2,6-fach erhöhtes Risiko zu sterben $(p<0,02)$. Bei weiterer Zunahme der Blastenanteile auf $>21 \%$ zeigte sich ein 5,7-fach erhöhtes Sterberisiko $(p<0,01)$. Auch die Zunahme der Aberrationszahl erwies sich im Hinblick auf das Gesamtüberleben als prognostisch ungünstig. Demnach hatten Patienten bei Nachweis von mehr als drei Aberrationen pro Fall ein 8,3-fach erhöhtes Sterberisiko $(p<0,01)$. Das Sterberisiko für Patienten mit klonaler Evolution war 3,6-mal so hoch wie das eines Patienten ohne klonale Evolution $(p=0,013)$. Die klonale Evolution stellte somit einen unabhängigen Risikofaktor für eine schlechte Prognose dar. Innerhalb der Evolutionsmuster hatten die Patienten aus den Gruppen „Genetische Regression“ (Evolutionsmuster C) und „Undulierender Verlauf“ (Evolutionsmuster E) nur ein geringes Sterberisiko, wobei ein Signifikanzniveau nicht erreicht wurde (HR Evolutionsmuster C 0,4, p=0,143; HR Evolutionsmuster E 0,6, p=0,665). Ein signifikanter Unterschied im Hinblick auf das Gesamtüberleben wurde ebenfalls nicht bei den Patienten aus den Gruppen „Genetische Stabilität“ (Evolutionsmuster B) und „Primäre Karyotypevolution“ (Evolutionsmuster D) festgestellt 
(HR Evolutionsmuster B 2,0, $p=0,081$; HR Evolutionsmuster D 1,9, $p=0,295)$. Wie bereits zuvor gezeigt wurde, wiesen Patienten aus der Gruppe "Genetische Stabilität" in 16,7\% der Fälle komplexe Anomalien auf (Vergleich 3.6.1.1). In dieser Gruppe wurden auch Patienten erfasst, die während des gesamten Verlaufs einen aberranten Karyotyp aufwiesen. Möglicherweise hatten diese Patienten bereits initial eine schlechte Prognose, die das Ergebnis dieser Gruppe erklären könnte.

Ein niedriger Hämoglobin-Wert $(<10 \mathrm{~g} / \mathrm{dl}$, HR 1,$8 ; p=0,055)$ und eine niedrige Leukozytenzahl im peripheren Blut $\left(<1,8^{*} 10^{3} / \mu l\right.$, HR 2,0; $\left.p=0,056\right)$ waren mit einem erhöhten Sterberisiko assoziiert. Das Signifikanzniveau wurde allerdings nicht erreicht. Das Alter, das Geschlecht sowie die Therapie hatten keinen signifikanten Einfluss auf das Gesamtüberleben.

Tabelle 13: Multivariate Analyse, Gesamtüberleben

\begin{tabular}{|c|c|c|c|c|c|c|}
\hline \multicolumn{7}{|c|}{ Gesamtüberleben } \\
\hline & & & & $95 \%$ Konfi & intervall & \\
\hline & & Anzahl & Hazard Ratio & unteres & oberes & $\mathrm{p}$-Werte \\
\hline \multirow{2}{*}{ Geschlecht } & Männer & 127 & \multirow{2}{*}{1,4} & \multirow{2}{*}{0,7} & \multirow{2}{*}{2,6} & \multirow{2}{*}{0,328} \\
\hline & Frauen & 75 & & & & \\
\hline Alter & & & 1,0 & 1,0 & 1,0 & 0,616 \\
\hline \multirow{2}{*}{ Therapie } & Nein & 8 & \multirow{2}{*}{0,6} & \multirow{2}{*}{0,1} & \multirow{2}{*}{4,8} & \multirow{2}{*}{0,646} \\
\hline & $\mathrm{Ja}$ & 194 & & & & \\
\hline \multirow{2}{*}{ Hämoglobin } & $\geq 10 \mathrm{~g} / \mathrm{dl}$ & 94 & \multirow{2}{*}{1,8} & \multirow{2}{*}{1,0} & \multirow{2}{*}{3,4} & \multirow{2}{*}{0,055} \\
\hline & $<10 \mathrm{~g} / \mathrm{dl}$ & 108 & & & & \\
\hline \multirow{2}{*}{ Leukozyten } & $\geq 1,8^{*} 10^{3} / \mu \mathrm{l}$ & 172 & \multirow{2}{*}{2,0} & \multirow{2}{*}{1,0} & \multirow{2}{*}{4,0} & \multirow{2}{*}{0,056} \\
\hline & $<1,8^{*} 10^{3} / \mu l$ & 30 & & & & \\
\hline \multirow{2}{*}{$\begin{array}{c}\text { Thrombo- } \\
\text { zyten }\end{array}$} & $\geq 100^{*} 10^{3} / \mu \mathrm{l}$ & 88 & \multirow{2}{*}{1,3} & \multirow{2}{*}{0,7} & \multirow{2}{*}{2,3} & \multirow{2}{*}{0,456} \\
\hline & $<100^{*} 10^{3} / \mu \mathrm{l}$ & 114 & & & & \\
\hline \multirow{5}{*}{$\begin{array}{c}\text { Blasten im } \\
\text { Knochen- } \\
\text { mark }\end{array}$} & $<5 \%$ & 110 & Referenzgruppe & & & $<0,01$ \\
\hline & $5-10 \%$ & 38 & 2,6 & 1,2 & 5,5 & 0,015 \\
\hline & $11-20 \%$ & 29 & 2,6 & 1,2 & 5,7 & 0,016 \\
\hline & $21-30 \%$ & 8 & 5,7 & 1,5 & 21,5 & $<0,01$ \\
\hline & $>30 \%$ & 17 & 4,0 & 1,6 & 10,0 & $<0,01$ \\
\hline \multirow{5}{*}{$\begin{array}{l}\text { Aberrationen } \\
\text { pro Fall }\end{array}$} & $\mathrm{A} / \mathrm{Fall}=0$ & 99 & Referenzgruppe & & & $<0,01$ \\
\hline & $\mathrm{A} / \mathrm{Fall}=1$ & 60 & 2,8 & 1,3 & 5,7 & $<0,01$ \\
\hline & A/Fall=2 & 15 & 0,8 & 0,2 & 3,1 & 0,763 \\
\hline & A/Fall=3 & 7 & 5,7 & 1,5 & 21,2 & $<0,01$ \\
\hline & A/Fall>3 & 21 & 8,3 & 2,5 & 28,2 & $<0,01$ \\
\hline \multirow{2}{*}{$\begin{array}{l}\text { klonale Evo- } \\
\text { lution (KE) }\end{array}$} & Nein & 153 & \multirow{2}{*}{3,6} & & & \\
\hline & $\mathrm{Ja}$ & 47 & & 1,3 & 9,8 & 0,013 \\
\hline & gen. Progression $(\mathrm{A})$ & 59 & Referenzgruppe & & & 0,055 \\
\hline klonale & gen. Stabilität (B) & 104 & 2,0 & 0,9 & 4,2 & 0,081 \\
\hline Evolutions- & gen. Regression (C) & 15 & 0,4 & 0,1 & 1,4 & 0,143 \\
\hline muster & primäre KE (D) & 17 & 1,9 & 0,6 & 6,5 & 0,295 \\
\hline & undulierend (E) & 7 & 0,6 & 0,1 & 5,3 & 0,665 \\
\hline
\end{tabular}




\subsubsection{AML-freies Überleben in der multivariaten Analyse}

In der multivariaten Analyse des AML-freien Überlebens konnte mit Zunahme der Blastenanteile im Knochenmark ein signifikant erhöhtes Risiko für eine AMLTransformation beobachtet werden $(5-10 \%, H R 3,3, p<0,01 ; 11-20 \%, H R 6,3, p<0,01$; $>21 \%, \mathrm{HR} 4,8, \mathrm{p}=0,05)$. Eine klonale Evolution stellte auch hier einen ungünstigen Prognoseparameter dar. So war das Risiko einer Progression in eine AML bei Patienten mit klonaler Evolution 4,6-fach höher als bei Patienten ohne klonale Evolution. Eine Zunahme der Aberrationszahl stellte keinen signifikanten Risikofaktor für eine AMLTransformation dar. Das Alter, das Geschlecht, die Therapie und die übrigen klinischen Parameter (Hämoglobin, Leukozyten und Thrombozyten) sowie die klonalen Evolutionsmuster zeigten ebenfalls keinen signifikanten Einfluss auf das AML-freie Überleben.

Tabelle 14: Multivariate Analyse, AML-freies Überleben

\begin{tabular}{|c|c|c|c|c|c|c|}
\hline \multicolumn{7}{|c|}{ AML-freies Überleben } \\
\hline & & \multirow[b]{2}{*}{ Anzahl } & \multirow[b]{2}{*}{ Hazard Ratio } & \multicolumn{2}{|c|}{ 95\% Konfidenzintervall } & \multirow[b]{2}{*}{$\mathrm{p}$-Werte } \\
\hline & & & & unteres & oberes & \\
\hline \multirow{2}{*}{ Geschlecht } & Männer & 112 & \multirow{2}{*}{1,6} & \multirow{2}{*}{0,8} & \multirow{2}{*}{3,2} & \multirow{2}{*}{0,148} \\
\hline & Frauen & 62 & & & & \\
\hline Alter & & & 1,0 & 1,0 & 1,0 & 0,748 \\
\hline \multirow{2}{*}{ Therapie } & Nein & 6 & & \multirow{2}{*}{0,0} & & \multirow{2}{*}{0,978} \\
\hline & $\mathrm{Ja}$ & 168 & & & & \\
\hline \multirow{2}{*}{ Hämoglobin } & $\geq 10 \mathrm{~g} / \mathrm{dl}$ & 84 & \multirow{2}{*}{1,2} & \multirow{2}{*}{0,6} & \multirow{2}{*}{2,3} & \multirow{2}{*}{0,551} \\
\hline & $<10 \mathrm{~g} / \mathrm{dl}$ & 90 & & & & \\
\hline \multirow{2}{*}{ Leukozyten } & $\geq 1,8^{*} 10^{3} / \mu l$ & 147 & \multirow{2}{*}{1,9} & \multirow{2}{*}{0,9} & \multirow{2}{*}{4,0} & \multirow{2}{*}{0,082} \\
\hline & $<1,8^{*} 10^{3} / \mu \mathrm{l}$ & 27 & & & & \\
\hline \multirow{2}{*}{$\begin{array}{l}\text { Thrombo- } \\
\text { zyten }\end{array}$} & $\geq 100 * 10^{3} / \mu \mathrm{l}$ & 81 & \multirow[b]{2}{*}{1,1} & \multirow[b]{2}{*}{0,6} & \multirow{2}{*}{1,9} & \multirow{2}{*}{0,864} \\
\hline & $<100^{*} 10^{3} / \mu \mathrm{l}$ & 93 & & & & \\
\hline \multirow{4}{*}{$\begin{array}{c}\text { Blasten im } \\
\text { Konchen- } \\
\text { mark }\end{array}$} & $<5 \%$ & 106 & Referenzgruppe & & & $<0,01$ \\
\hline & $5-10 \%$ & 36 & 3,3 & 1,6 & 7,1 & $<0,01$ \\
\hline & $11-20 \%$ & 27 & 6,3 & 3,0 & 13,4 & $<0,01$ \\
\hline & $21-30 \%$ & 5 & 4,8 & 1,0 & 23,4 & 0,050 \\
\hline \multirow{5}{*}{$\begin{array}{l}\text { Aberrationen } \\
\text { pro Fall }\end{array}$} & A/Fall=0 & 89 & Referenzgruppe & & & 0,662 \\
\hline & $\mathrm{A} / \mathrm{Fall}=1$ & 52 & 1,4 & 0,6 & 3,1 & 0,386 \\
\hline & A/Fall=2 & 12 & 0,9 & 0,3 & 3,5 & 0,933 \\
\hline & $\mathrm{A} / \mathrm{Fall}=3$ & 6 & 1,3 & 0,3 & 6,4 & 0,721 \\
\hline & A/Fall >3 & 15 & 2,2 & 0,5 & 9,3 & 0,270 \\
\hline \multirow{2}{*}{$\begin{array}{l}\text { klonale Evo- } \\
\text { lution (KE) }\end{array}$} & Nein & 135 & 46 & 16 & & \\
\hline & $\mathrm{Ja}$ & 39 & 4,6 & 1,6 & 13,1 & $<0,01$ \\
\hline & gen. Progression $(A)$ & 52 & Referenzgruppe & & & 0,415 \\
\hline klonale & gen. Stabilität (B) & 87 & 1,0 & 0,5 & 2,1 & 0,941 \\
\hline Evolutions- & gen. Regression (C) & 15 & 1,0 & 0,3 & 3,5 & 0,939 \\
\hline muster & primäre KE (D) & 14 & 0,2 & 0,0 & 1,0 & 0,057 \\
\hline & undulierend $(E)$ & 6 & 1,3 & 0,3 & 6,4 & 0,757 \\
\hline
\end{tabular}




\section{Diskussion}

\section{Diskussion}

Das Ziel dieser retrospektiven Studie war es, die prognostische Bedeutung der klonalen Evolution und der Evolutionsmuster bei Patienten mit MDS zu untersuchen. Dabei wurden Daten ausgewertet, die bei deren Erstuntersuchung und bei Verlaufskontrollen erhoben wurden. Bislang liegen zu dieser Thematik nur wenige Studien vor, in denen teilweise nur kleine Patientenkollektive und nicht immer hinreichend viele Verlaufsuntersuchungen durchgeführt wurden. Insbesondere die Verlaufsbeobachtungen sind jedoch wichtig, um die unterschiedlichen Evolutionsmuster herauszuarbeiten. Erstmals wurden von Tricot et al. 1985 unterschiedliche Evolutionsmuster und deren klinische Verläufe für 46 MDSPatienten unter Berücksichtigung hauptsächlich der Blastenanteile beschrieben. Bei der Durchsicht der Literatur findet sich eine Studie (Haferlach et al. 2011), in der die prognostische Bedeutung der klonalen Evolution an einem größeren Kollektiv von MDS-Patienten als in der hier vorliegenden Arbeit untersucht wurde (Tabelle 15). Die unterschiedlichen Evolutionsmuster wurden jedoch in dieser Studie nicht berücksichtigt. Somit stellt die vorliegende Arbeit einen wesentlichen Beitrag insbesondere zur Klärung der prognostischen Bedeutung der klonalen Evolutionsmuster bei Patienten mit MDS dar.

Tabelle 15: Studien zu klonaler Evolution

\begin{tabular}{lccc}
\hline \multicolumn{1}{c}{ Autoren } & Jahr & Untersuchte Pat. & Untersuchte Pat. mit Verläu- \\
fen
\end{tabular}

Die Inzidenz der klonalen Evolution lag in der vorliegenden Arbeit bei 12,9\%. Bei Betrachtung der nur aberranten Fälle mit NA-Verteilung (normale und aberrante Metaphasen im Mosaik) und AA-Verteilung (ausschließlich aberrante Metaphasen) fand sich eine Inzidenz von $25,8 \%$. In den bisher veröffentlichten Studien zeigte sich eine große Spannbreite der 
Inzidenz der klonalen Evolution von 7,6\% (White et al. 1994) bis 39,0\% (Tien et al. 1995). In den 225 durchgeführten zytogenetischen Verlaufsuntersuchungen fand sich eine Inzidenz der klonalen Evolution von 8\%. Die mediane Beobachtungszeit bis zum Nachweis einer klonalen Evolution betrug 11,9 Monate. Horiike et al. (1988) fanden in einer sequentiellen Untersuchung von 30 MDS-Patienten bei 10 Patienten (33,3\%) eine klonale Evolution. In einer weiteren Studie mit einem größeren Kollektiv von 77 Patienten mit Verlaufsuntersuchungen fand sich in 12 Fällen (15,6\%) eine klonale Evolution. Die mediane Beobachtungszeit betrug 12 Monate (Geddes et al. 1990). Mit einer längeren medianen Beobachtungszeit von 45,2 Monaten wurde bei 153 MDS-Patienten in 30,7\% der Fälle eine klonale Evolution nachgewiesen (Bernasconi et al. 2010). Mögliche Ursache für diese großen Unterschiede kann die unterschiedlich lange mediane Beobachtungszeit sein. In diesem Zusammenhang vermuteten Haase et al. (2011) als weitere Ursache auch die unterschiedlich großen Patientenkollektive.

Frauen wiesen mit 16\% signifikant häufiger als Männer (10,7\%) eine klonale Evolution auf $(p=0,037)$. Diese Geschlechtsdifferenz wurde bereits in der Dissertation von Frau Dr. Schanz (2003) beschrieben und konnte durch die hier vorliegende Arbeit bestätigt werden. In der übrigen Literatur ist eine derartige Geschlechtsdifferenz nicht zu finden. Frauen und Männer erkranken generell gleich häufig an MDS. Eine Ausnahme zeigt sich beim Auftreten einer 5q-Deletion. Frauen erkranken dann 1,5-fach häufiger im Vergleich zu Männern. (Boultwood et al. 1994, Van den Berghe und Michaux 1997, Giagounidis et al. 2004). In der vorliegenden Studie wiesen Patienten mit klonaler Evolution signifikant häufiger eine $5 q$-Deletion auf als Patienten ohne klonale Evolution $(p<0,01)$. Pedersen zeigte 1998 in einer Meta-Analyse, dass Frauen mit einer 5q-Deletion überwiegen, bedingt dadurch, dass Frauen durchschnittlich länger als Männer mit einer entsprechenden Deletion überleben.

Die Auswertung der Überlebensdaten in der vorliegenden Arbeit belegt, dass die klonale Evolution mit einer ungünstigen Prognose einhergeht. Die mediane Überlebenszeit der Patienten mit klonaler Evolution betrug 24,4 Monate $(n=72)$. Bei Patienten ohne klonale Evolution dagegen betrug diese 103,6 Monate $(n=421, p<0,01)$. In früheren Studien konnte die verkürzte Überlebenszeit von Patienten mit klonaler Evolution anhand kleinerer Fallzahlen ebenfalls beobachtet werden. So wurde in der Studie von Horiike et al. (1988) gezeigt, dass 10 Patienten mit klonaler Evolution im Median eine signifkant kürzere Über- 
lebenszeit aufwiesen als 20 Patienten ohne klonale Evolution (2,5 Monate vs. 20 Monate) Ebenfalls konnten Geddes et al. (1990) und White et al. (1994) eine signifikant kürzere Überlebenszeit bei 12 bzw. 15 MDS-Patienten mit klonaler Evolution nachweisen. In einer Studie von Wang et al. (2010) betrug die mediane Überlebenszeit von Patienten mit Karyotypevolution ( $n=18)$ 25,8 Monate. Patienten ohne klonale Evolution ( $n=67)$ zeigten eine um 19,6 Monate verlängerte Überlebenszeit $(p<0,01)$. In einer aktuellen Untersuchung eines Kollektivs von 2902 MDS-Patienten von Schanz et al. (2012) konnte bei Betrachtung der nur aberranten Fälle ebenfalls eine verkürzte Überlebenszeit mit nur 11,4 Monaten bei klonaler Evolution im Vergleich zu 28,9 Monaten $(p<0,01)$ bei fehlender klonaler Evolution beobachtet werden.

Darüber hinaus konnten in der vorliegenden Arbeit zwei weitere wesentliche Aspekte der prognostischen Relevanz der klonalen Evolution herausgearbeitet werden. So zeigte sich, dass die Prognose davon abhängt, ob bereits bei der Erstdiagnose eine klonale Evolution vorliegt und falls nicht, wie schnell diese im Erkrankungsverlauf eintritt. Es wurde festgestellt, dass Patienten mit Nachweis einer klonalen Evolution bereits bei Erstdiagnose eine kürzere Überlebenszeit aufwiesen als Patienten, die erst im Krankheitsverlauf eine solche entwickelten (8,2 Monate vs. 32,6 Monate, $p<0,01$ ). Die klonale Evolution ist ein Kriterium, welches die Dynamik und damit den Progress der Erkrankung widerspiegelt (Haase 2005). Es ist davon auszugehen, dass bei Patienten mit klonaler Evolution zum Zeitpunkt der Erstdiagnose bereits ein maximal maligner Zellklon vorlag. Die Patienten hatten sich möglicherweise hier schon in einem fortgeschrittenen Krankheitsstadium befunden und die verschiedenen Evolutionsschritte konnten somit in einer einzigen zytogenetischen Untersuchung erfasst werden. Dies erklärt die ungünstige Prognose der Patienten mit einer klonalen Evolution bereits bei Erstdiagnose. Es wurde darüber hinaus untersucht, ob die Prognose vom Zeitpunkt des Auftretens der klonalen Evolution innerhalb der sequentiellen zytogentischen Untersuchungen abhängt. Es konnten zwei Gruppen unterschieden werden. Zum einen eine Gruppe, bei der sehr rasch eine klonale Evolution nachgewiesen wurde (5,3 Monate) und zum anderen eine Gruppe, die über einen längeren Zeitraum hinweg erst eine Karyotypevolution entwickelte (21,9 Monate, $p<0,01)$. Zwischen diesen beiden Gruppen fanden sich auch Unterschiede in den medianen Überlebenszeiten. So wiesen Patienten mit früher Karyotypevolution (<365 d, Vergleich 3.2.4) eine mediane Überlebenszeit von 13,25 Monaten auf, während die mediane Überlebenszeit bei Patienten mit einer späten Evolution ( $>365$ d) 32,56 Monate betrug $(p=0,061)$. In einer früheren 
retrospektiven Studie von Ohyashiki et al. (1995) konnte in 15 Fällen ebenfalls ein Unterschied im zeitlichen Auftreten einer klonalen Evolution nachgewiesen werden (KE $<100 \mathrm{~d}$ vs. KE >100 d nach Erstdiagnose). Patienten, die innerhalb von 100 Tagen eine klonale Evolution entwickelten, wiesen komplexe Aberrationen auf. Innerhalb der klonalen Evolution kann hinsichtlich des zeitlichen Auftretens weiter differenziert werden und das frühe Auftreten ( $<365$ d) der klonalen Evolution ist mit einer verkürzten Überlebenszeit assoziiert. Ferner ist der Diagnosezeitpunkt der klonalen Evolution von prognostischer Bedeutung. Dies sollte Anlass geben, dieses Phänomen an größeren Patientenkollektiven weiter zu überprüfen.

In der vorliegenden Arbeit konnte nicht nur die prognostische Bedeutung der klonalen Evolution für die Überlebenszeit, sondern auch für die AML-freie Überlebenszeit gezeigt werden. Bereits Bernasconi et al. ermittelten ein 36-fach erhöhtes Risiko einer AMLTransformation bei Patienten mit klonaler Evolution mittels univariater CoxRegressionsanalyse (Bernasconi et al. 2010). Auch in älteren Publikationen wurde, trotz geringer Fallzahlen, ein erhöhtes Risiko für das Auftreten einer AML bei Vorliegen einer klonalen Evolution beschrieben (Horiike et al. 1988, Geddes et al. 1990, Wang et al. 2010). In der hier vorliegenden Arbeit konnte dieses Ergebnis nun mit einer größeren Fallzahl reproduziert werden. So entwickelten mehr als doppelt so viele Patienten mit klonaler Evolution eine AML als Patienten ohne klonale Evolution ( $48,0 \%$ vs. $21,4 \%, p<0,01$ ). Während die AML-freie Überlebenszeit für Patienten mit klonaler Evolution bei einem Median von 17,5 Monaten lag, konnte das 50\%- und auch das 25\%-Perzentil für Patienten ohne klonale Evolution nicht erreicht werden $(p<0,01)$. In einer Studie von Schanz et al. (2012), in der ausschließlich aberrante Fälle untersucht wurden, wurde ebenfalls eine verkürzte AML-freie Überlebenszeit bei Vorliegen einer klonalen Evolution festgestellt (33 Monate vs. 121,2 Monate, $p<0,01)$. In der vorliegenden Arbeit wurde darüber hinaus der Frage nachgegangen, ob ein Übergang in eine AML bei Patienten mit früher bzw. später klonaler Evolution miteinander korreliert. Dieser Zusammenhang ist neu und wurde unseres Wissens bisher von keiner anderen Arbeitsgruppe untersucht. Diesbezüglich konnten jedoch, trotz großer Differenzen hinsichtlich der AML-freien Überlebenszeit (6,8 Monate vs. 20,3 Monate), keine signifikanten Unterschiede zwischen den Patienten mit früher und später klonaler Evolution gefunden werden $(p=0,242)$. 
Das Auftreten einer klonalen Evolution korreliert eng mit komplexen Anomalien durch das Hinzukommen weiterer Aberrationen und Akkumulation von Aberrationen (Haase 2005, Haase 2008). Bei Patienten mit klonaler Evolution lag eine hohe Aberrationsrate mit Nachweis von mehr als drei Aberrationen in 43,6\% der Fälle vor. Dies impliziert, dass zytogenetische Aberrationen bei klonaler Evolution kumulieren. Zusätzliche Aberrationen treten bei der klonalen Evolution häufiger in Form von komplexen Aberrationen auf. Patienten mit klonaler Evolution wiesen mit 54,3\% doppelt so häufig komplexe Anomalien auf wie Patienten ohne klonale Evolution $(p<0,01)$. Dass die klonale Evolution mit einer Zunahme der Komplexität einhergeht, konnte ebenfalls in einer Arbeit von Haferlach et al. (2011) gezeigt werden. Darüber hinaus war die klonale Evolution häufiger mit einem Übergang in eine höhere Gruppe des IPSS-Risikoscores verbunden. Bei Patienten mit klonaler Evolution und zusätzlich komplex aberrantem Karyotyp fand sich in der vorliegenden Arbeit eine verkürzte Überlebenszeit von 3,8 Monaten. Diese verlängerte sich bei diesen Patienten ohne den Nachweis eines komplexen Karyotyps auf 32,6 Monate ( $p<0,01$, Vergleich 3.3.2). Aus der Literatur ist bereits bekannt, dass die Anzahl der Aberrationen sowie die Komplexität des Karyotyps einen ungünstigen Einfluss auf die mediane Überlebenszeit haben (Haase et al. 2007, Haase 2008, Schanz et al. 2012). In der vorliegenden Arbeit konnte veranschaulicht werden, dass sich die klonale Evolution bei Vorliegen eines komplexen Karyotyps zusätzlich negativ auf die mediane Überlebenszeit auswirkt und sich diese dadurch weiter verkürzt.

Eine semiquantitative Angabe für die Zunahme des aberranten Klons ist der AnomalieVerteilungstyp (Schanz 2003, Haase 2005, Bloomfield 1984). Unterschieden werden dabei drei Kategorien: NN-Karyotyp (ausschließlich normale Metaphasen), NA-Karyotyp (normale und aberrante Metaphasen im Mosaik) und AA-Karyotyp (ausschließlich aberrante Metaphasen). Einige Studien konnten bereits die prognostische Relevanz der Zunahme der Klongröße bei MDS-Patienten aufzeigen (Geddes et al. 1990, González Manso et al. 1992, White et al. 1994, Schanz 2003, Haase 2005). Unter Einbeziehung der klonalen Evolution konnte in der vorliegenden Arbeit eine neuartige, erweiterte Subgruppeneinteilung des Anomalie-Verteilungstyps vorgenommen werden. So konnten neben den bisher beschriebenen drei Subgruppen zwei weitere Gruppen definiert werden. Zwei wesentliche Aspekte sind hieraus ersichtlich geworden: Die Prognose wird zum einen durch die Zunahme der Klongröße und zum anderen durch das Hinzukommen von zusätzlichen Aberrationen beeinflusst. So zeigte sich bei Patienten mit einer klonalen Evolution, dass die 


\section{Diskussion}

mediane Überlebenszeit bei Vorliegen einer Klongröße von 100\% mit 6,6 Monaten signifikant kürzer war als bei Vorliegen einer NA-Verteilung (Klongröße 1-99\%) mit 18,0 Monaten $(p=0,046)$. Auch bei den ausschließlich aberranten Karyotypen (AA) zeigte sich eine signifikante Verkürzung der medianen Überlebenszeit bei Vorliegen einer klonalen Evolution um 12,9 Monate (6,6 Monate vs. 19,5 Monate, $p=0,022)$. Innerhalb der Gruppe der Patienten mit Karyotypevolution bleibt der Einfluss des Verteilungstyps hinsichtlich der Prognose bestehen und erlaubt die Einteilung weiterer klinischer Subgruppen.

Weiterhin wurde die Inzidenz distinkter zytogenetischer Aberrationen innerhalb der Gruppe der Patienten mit klonaler Evolution und darüber hinaus auch innerhalb der verschiedenen zytogenetischen Evolutionsmuster untersucht. Betrachtet wurden die häufigsten Anomalien (Deletion 5q, Monosomie 7/Deletion 7q, Trisomie 8, Deletion 20q, 17p-Anomalien und der Y-Verlust). In der Literatur wurde bereits beschrieben, dass bei $5 q$-Anomalien durch zusätzliche einzelne Anomalien und multiple Anomalien die Überlebenszeit der Patienten signifikant verkürzt wird (Giagounidis et al. 2006, Haase et al. 2007, Haase 2008, Schanz et al. 2012). In der vorliegenden Arbeit konnte gezeigt werden, dass Patienten mit einer klonalen Evolution signifikant häufiger $5 q$-Veränderungen aufweisen als Patienten ohne klonale Evolution (46,7\% vs. 25,0\%, p<0,01). Benítez et al. berichteten, dass in der Mehrschrittpathogenese der MDS 5q-Anomalien mit einer Expansion des aberranten Zellklons einhergehen (Benítez et al. 1987). Darüber hinaus wird diskutiert, dass molekulargenetisch zwei benachbarte Regionen, eine zentromernahe und eine mehr telomerwärts gelegene Region, innerhalb der Bande $5 q 31$ existieren. Abhängig von der molekulargenetischen Region ist der klinische Verlauf mit einer ungünstigen Prognose (zentromernahe Region) oder einer günstigen Prognose (telomernahe Region) assoziiert (Nagarajan 1995, Boultwood et al. 2002, Olney et al. 2002, Pellagatti et al. 2006, Haase 2008). Die Hypothese der Mehrschrittpathogenese der klonalen Evolution und die Bedeutung der 5q-Deletion, die zu einer Expansion des aberranten Zellklons führt, wird durch unsere Daten unterstützt. Auch in einer deutsch-österreichischen Studie (Steidl et al. 2005) wurde eine Korrelation zwischen der Karyotypevolution und der 5q-Deletion beobachtet. In dieser Studie wurde bei 155 Patienten das Auftreten zusätzlicher Chromosomenanomalien, die Zunahme der Klongröße auf $>20 \%$ und die Entstehung eines komplett aberranten Karyotyps (AA) bei initialem Mosaik-Karyotyp (NA) untersucht. Auch hier stellte die 5q-Deletion die häufigste Anomalie bei klonaler Evolution dar. In einer aktuell publizierten Studie von Zemanova et al. (2014) wurden 157 MDS-Patienten mit komplexen Anoma- 
lien untersucht. In 47\% der Fälle wurden Chromosomenumbauten, möglicherweise als Folge einer „Chromothripsis“, gefunden. In diesem Zusammenhang zeigte sich, dass die $5 q$-Deletion instabil war und häufig in Kombination mit unterschiedlichen unbalancierten Anomalien vorlag. Diese Patienten wiesen eine sehr ungünstige Prognose auf.

Die Monosomie 7/7q-Deletion erwies sich in der vorliegenden Arbeit als ungünstige IndexAberration innerhalb der klonalen Evolution. Gemäß der Literatur sind Monosomie 7/7qDeletion als prognostisch „ungünstig“ für MDS-Patienten eingestuft (Haase 2008, Schanz et al. 2012). Die zugrunde liegende Arbeit zeigte, dass die mediane Überlebenszeit von Patienten mit klonaler Evolution, welche eine Monosomie 7/7q-Deletion aufwiesen, kürzer war als von denjenigen, bei denen die Monosomie 7/7q-Deletion nicht als Index-Aberration vorlag (8,2 Monate vs. 18,2 Monate, $p=0,020)$. In einer Studie von Jabbour et al. (2013) wurde bei MDS-Patienten das Auftreten von Chromosomenanomalien im Erkrankungsverlauf bei initial normalem Karyotyp oder innerhalb eines bereits aberranten Karyotyps gemäß Shaffer et al. (2013) untersucht. Dies war bei 107 von 365 Patienten (29\%) der Fall. Bei 20\% traten Chromosom 7 Anomalien und in 15\% der Fälle eine Trisomie 8 auf. Ein gehäuftes Auftreten einer Trisomie 8 bei klonaler Evolution konnte in der vorliegenden Studie nicht eruiert werden. So fand sich bei $20,7 \%$ der Patienten mit und bei 17,4\% der Patienten ohne klonale Evolution eine Trisomie 8. In der Literatur wird die Trisomie 8 als eine häufige sekundäre Anomalie bei Patienten mit klonaler Evolution beschrieben (Steidl et al. 2005). Die Tabelle 16 fasst eine Liste mit den häufigsten primären Anomalien und sekundären Anomalien bei MDS-Patienten zusammen (Haase et al. 2011).

Tabelle 16: Primäre und sekundäre Aberrationen bei MDS gemäß Johansson et al. 1996, Mallo et al. 2011, Steidl et al. 2005 (Haase et al. 2011, S. 64. Mit freundlicher Genehmigung des Verlags)

\begin{tabular}{cc}
\hline primäre Aberrationen & assoziierte sekundäre Aberrationen \\
\hline $\operatorname{der}(1 ; 7)(\mathrm{p} 10 ; \mathrm{q} 10)$ & +8 \\
\hline $\operatorname{der}(3 \mathrm{q} 21 / \mathrm{q} 26)$ & -7 \\
\hline $\operatorname{del}(5 \mathrm{q})$ & $-7,+8, \operatorname{del}(12 \mathrm{q}), \operatorname{der}(17 \mathrm{p}),+21,-18 / 18 \mathrm{q}-,-\mathrm{Y}$ \\
\hline-7 & $\operatorname{del}(20 \mathrm{q}),+8,+21$ \\
\hline+8 & $\operatorname{del}(5 \mathrm{q}),-7,+8,-17 / \operatorname{der}(17 \mathrm{p}),+22,-\mathrm{Y}$ \\
\hline $\operatorname{del}(20 \mathrm{q})$ & $-7 / \operatorname{del}(7 \mathrm{q}),+8,+21$ \\
\hline
\end{tabular}

Hinsichtlich der primären und sekundären Anomalien wurde in der vorliegenden Arbeit deskriptiv die Zytogenetik der Patienten, bei denen im Krankheitsverlauf eine klonale Evolution nachgewiesen wurde, analysiert (Tabelle 17). 
Diskussion

Tabelle 17: Primäre und sekundäre Aberrationen bei klonaler Evolution, eigene Daten

\begin{tabular}{cc}
\hline primäre Aberrationen & assoziierte sekundäre Aberrationen \\
\hline $\operatorname{der}(1 ; 7)(\mathrm{p} 10 ; \mathrm{q} 10)$ & +8 \\
\hline $\operatorname{del}(20 \mathrm{q})$ & $-7 / 7 \mathrm{q}-,+8,-\mathrm{Y}$ \\
\hline & $+4,-20,-21, \operatorname{del}(16)(12.1), \mathrm{t}(2 ; 11)(\mathrm{p} 21 \mathrm{p} 23)$, del \\
$5 \mathrm{q}-$ & $(8)(\mathrm{q} 22), \operatorname{der}(12) \mathrm{t}(12 ; 20)(\mathrm{p} 12 ; ?), \operatorname{der}(13)$ \\
$\mathrm{t}(13 ; 18)(\mathrm{p} 11 ; \mathrm{q} ?), \operatorname{del}(18)(\mathrm{q} 12)$
\end{tabular}

Bei den hier erfassten Fällen mit 5q-Deletionen waren die nachgewiesenen sekundären Anomalien besonders heterogen. Häufig fanden sich bei Patienten mit einer $5 q$-Anomalie unbalancierte strukturelle Anomalien. Die in der Tabelle 16 zusammengefassten Daten aus den Publikationen von Johansson et al. (1996), Mallo et al. (2011), Steidl et al. (2005) für die primären Aberrationen $\operatorname{der}(1 ; 7)(p 10 ; q 10)$ und del(20q) konnte in der vorliegenden Arbeit bei Patienten mit klonaler Evolution reproduziert werden. Beachtet werden muss, dass bei MDS-Patienten aufgrund der zytogenetischen Vielfalt auch unterschiedliche Kombinationen von Aberrationen vorliegen (Haase 2008). Aus publizierten Daten ist bekannt, dass komplexe Anomalien mit unbalancierten strukturellen Anomalien, wie beispielsweise am Chromosom $5 q$ und $7 q$, assoziiert sind und mit einer größeren Häufigkeit bei Patienten mit einer klonalen Evolution auftreten (Haase 2008). Die Arbeitsgruppe von Prof. Dr. med. D. Haase (Laborleitung Diagnostiklabore, UMG) hat in diesem Zusammenhang das Schema zur Darstellung der Evolutionsmuster von Tricot et al. (1985) weiterentwickelt (Abbildung 37). Eine Progression der Erkrankung und eine schlechtere Prognose sind mit Veränderungen des p53-Gens assoziiert und treten bei ca. 10\% der MDSPatienten auf (Lai et al. 1995, Sebaa et al. 2012). Darüber hinaus zeigten Studien, dass die Mutation des p53-Gens häufig bei komplexen Karyotypen mit 5q-Deletionen und 17pDeletionen vorliegt (Sebaa et al. 2012, Martínez-Ramírez et al. 2004). Auch zeigten Volkert et al. (2014) in einer Studie mit 1200 MDS- und AML- Patienten, dass 5q-Deletionen häufiger mit klonaler Evolution und einer p53-Mutation assoziiert waren. Diese Patienten erlebten einen raschen Erkrankungsprogress. In der hier vorliegenden Arbeit konnte neben dem häufigen Auftreten einer $5 q$-Deletion bei klonaler Evolution auch gezeigt werden, dass auch 17p-Deletionen mit klonaler Evolution assoziiert sind ( $5,4 \%$ vs. $1,1 \%, p=0,030$ ). Weiterhin erwies sich die 17p-Deletionen als prognostisch sehr ungünstig, wenn diese bei Patienten mit klonaler Evolution auftraten (1,8 Monate vs. 17,9 Monate, $p=0,002)$. 


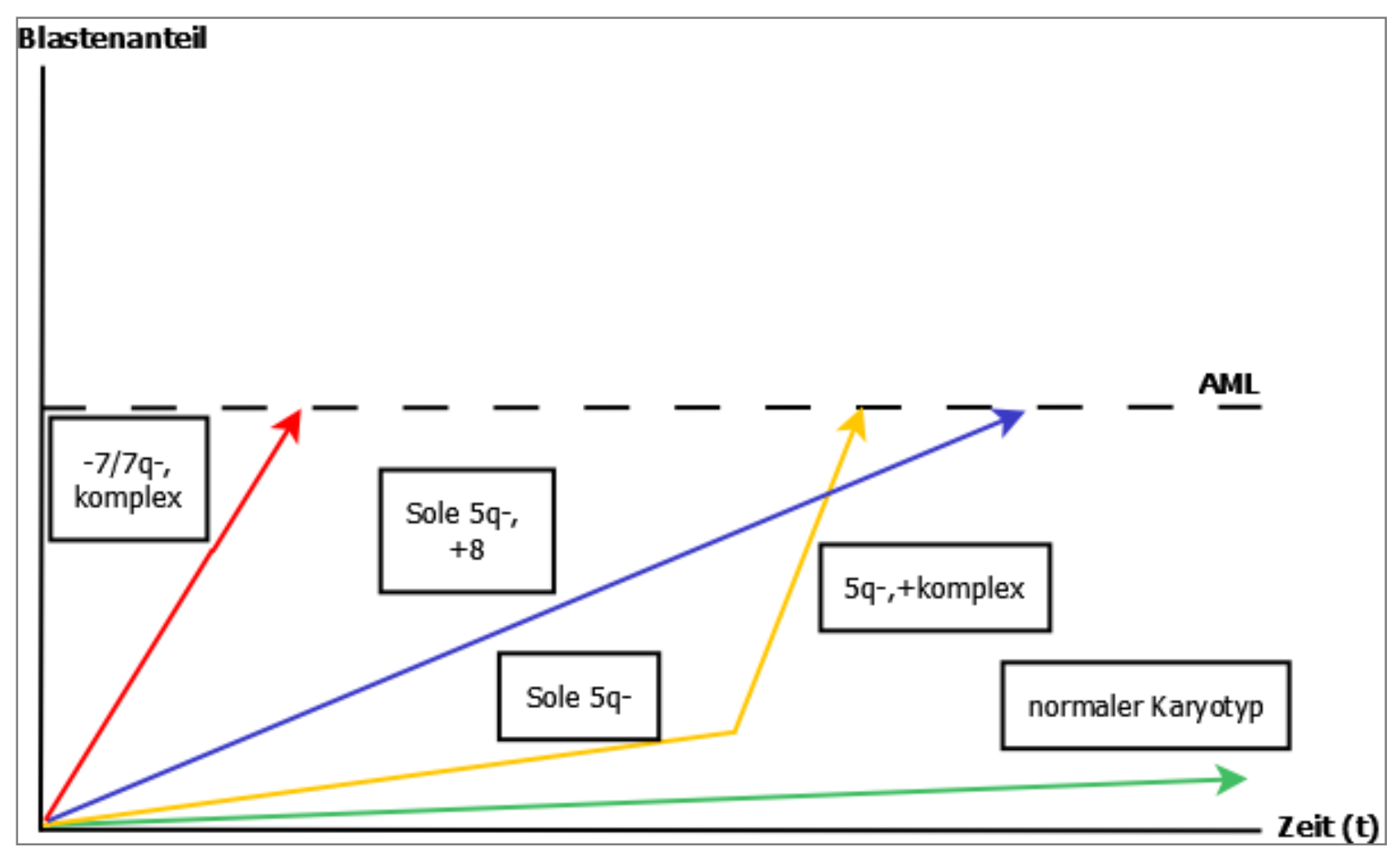

Abbildung 37: Muster der klonalen Evolution gemäß Tricot et al. 1985, modifiziert (Haase et al. 2011 S. 65. Mit freundlicher Genehmigung des Verlags)

Die älteste publizierte Studie, die zu Evolutionsmustern durchgeführt wurde, stammt von Tricot et al. (1985). 46 Patienten mit zytogenetischen Verlaufsuntersuchungen wurden hinsichtlich ihrer klinischen Verläufe in vier Gruppen eingeteilt. Bei der Einteilung wurde im Wesentlichen der Blastenanteil im Knochenmark berücksichtigt. In der vorliegenden Arbeit wurde die Einteilung der Evolutionsmuster erweitert und modifiziert. Anhand von 280 Patientendaten (Daten zur klonalen Evolution bei Erstdiagnose und 225 Verlaufsdaten) erfolgte eine Einteilung in unterschiedliche klinische Verläufe. Dabei wurde die prognostische Relevanz unterschiedlicher klonaler Evolutionsmuster untersucht. Die meisten Patienten ( $n=126,45,0 \%$ ) wurden in der Gruppe "Genetische Stabilität" erfasst. Es zeigte sich, dass diese Patienten mit im Median 186,8 Monaten die längste Überlebenszeit aufwiesen. 73 Patienten $(26,1 \%)$ bildeten die Gruppe „Genetische Progression“ und hatten eine mediane Überlebenszeit von 47,2 Monaten. Die Gruppe "Genetische Regression“ bestand aus 18 Patienten. Die mediane Überlebenszeit in dieser Gruppe lag bei 37,5 Monaten. Eine mögliche Erklärung für die schlechtere Prognose dieser Patienten war möglicherweise ein frühzeitiger Therapiebeginn. Es ist anzunehmen, dass diese Patienten initial eine ungünstigere klinische Prognose und damit einen Therapiebedarf aufgewiesen haben. 94,4\% der Patienten wurden therapiert. In 35,3\% der Fälle erhielten die Patienten eine Chemotherapie. Eine deutliche Verkürzung der medianen Überlebenszeit auf nur 3,6 Monate wurde 
bei den Patienten aus der Gruppe „Primäre Karyotypevolution“ festgestellt. Die beste Prognose ergab sich für Patienten aus der Gruppe mit undulierendem Verlauf. Hier konnte das 50\%- und auch das 25\%-Perzentil nicht erreicht werden. Diese Unterschiede erreichten ein hochsignifikantes Niveau. Dies wurde in der Literatur bisher so nicht beschrieben. Allerdings wurde in einer aktuellen Studie von Bochtler et al. (2013) auch unterschiedliche Subklonmuster/Evolutionsmuster bei Patienten mit AML herausgearbeitet. Auch hier wurden Daten konventioneller Karyotypisierung der Metaphasechromosomen verwendet. Subklone wurden bei 418 von 2639 Patienten gefunden (15,8\%). Bei Berücksichtigung nur der aberranten Fälle waren es 33\% (418/1266). Die Subklonhäufigkeit lag bei zytogenetischen Hochrisikopatienten mit knapp 70\% noch höher. Anhand der Anzahl der Subklone (2-5) stellten Bochtler et al. schematisch Evolutionsmuster dar. In dieser Studie wurde auch gezeigt, dass bei Übergang eines MDS in eine AML mindestens ein Subklon vorlag. In einer Studie von Braulke et al. (2013) konnte gezeigt werden, dass das Auftreten einer klonalen Evolution bei MDS auch mittels FISH-Analyse (fluorescence in situ hybridization) von CD34+ Stammzellen aus dem peripheren Blut erkannt werden kann. Bei 9 von den 385 untersuchten Patienten konnte die klonale Evolution allerdings nur in der konventionellen zytogenetischen Untersuchung und nicht in der FISH-Analyse nachgewiesen werden. Folgende Aberrationen wurden in diesem Zusammenhang häufig gefunden: 7/del(7q), del(20q), del(21q)/-21/+21, del(5q), del(17p), +8, Y-Verlust.

In einer multizentrischen Studie von Steidl et al. (2005) wurden über 500 Patienten hinsichtlich der Karyotypevolution in drei Gruppen eingeteilt. Hierbei wurden folgende Subgruppen unterschieden: Auftreten von zusätzlichen Aberrationen zu bereits vorhandenen Aberrationen, Klongrößenzunahme um $>20 \%$ und komplett aberranter Karyotyp bei initialem Mosaik-Karyotyp. Dies entspricht in der vorliegenden Arbeit der Gruppe „Genetische Progression“. In dieser Studie wurde herausgearbeitet, dass Patienten die einen initial normalen Karyotyp hatten und schließlich im Verlauf ihrer Erkrankung eine Karyotypevolution entwickelten, eine schlechte Prognose hatten. In einer Studie von Marisavljevic et al. (2013) zum Thema Karyotypevolution wiesen die untersuchten 76 MSD-Patienten initial nahezu ebenso häufig einen normalem Karyotyp wie aberrante Metaphasen (24,5\% vs. $26,1 \%$ ) auf. Es fanden sich auch keine signifikanten Unterschiede zwischen diesen beiden Gruppen hinsichtlich des Gesamtüberlebens (60 Monate vs. 35 Monate, $p=0,06$ ) und der Transformation in eine akute Leukämie (49 Monate vs. 32 Monate, $\mathrm{p}=0,26)$. Von diesen Autoren wurden daher die bisher veröffentlichten Daten zur prog- 
nostischen Bedeutung der klonalen Evolution (Wang et al. 2010, Geddes et al. 1990, Horiike et al. 1988) kritisch hinterfragt. Allerdings ist dabei anzumerken, dass die Aussagekraft der Studie von Marisavljevic et al. (2013) aufgrund ihrer geringen Fallzahl limitiert ist. In Übereinstimmung mit unseren Ergebnissen belegen die bisher veröffentlichten Studien überwiegend, dass bei Patienten mit MDS die klonale Evolution mit einer ungünstigen Prognose in Hinblick auf das Gesamtüberleben und den Übergang in eine AML assoziiert ist. In der vorliegenden Arbeit konnte in der mutlivariaten Analyse darüber hinaus gezeigt werden, dass die klonale Evolution einen wichtigen unabhängigen Prognosefaktor darstellt.

\subsection{Methodenkritik}

Ein generelles Problem bei retrospektiven Studien stellt die lückenlose Erfassung von Patientendaten dar.

Bei den insgesamt 225 erfassten zytogenetischen Verlaufsdaten ergaben sich im Rahmen der weiteren Einteilungen, wie beispielweise in die unterschiedlichen Evolutionsmuster, zum Teil nur kleine Subgruppen. Die Ergebnisse dieser Studie sollten daher in größeren Patientenkollektiven mit entsprechenden zytogenetischen Verlaufsdaten vertieft werden.

Bei der Datenerhebung zum Zeitpunkt der Erstdiagnose fanden sich ebenfalls kleinere Lücken, die aufgrund der Größe des Patientenkollektivs von 729 Patienten wenig ins Gewicht fielen.

Dennoch konnte insgesamt ein wesentlicher Beitrag zur Beurteilung der prognostischen Relevanz der klonalen Evolution und der Evolutionsmuster bei Patienten mit MDS herausgearbeitet werden.

\subsection{Ausblick}

Die Zytogenetik stellt einen wichtigen unabhängigen Prognosefaktor bei Patienten mit MDS dar (Greenberg et al. 1997, Schanz et al. 2012). Die Patienten weisen sehr heterogene Krankheitsverläufe auf, die von einem über viele Jahre anhaltenden stabilen Verlauf zu einem schnellen Progress bis hin zur Transformation in eine AML reichen (Germing und Haas 2009). Bei etwa 50\% der erkrankten Patienten finden sich in der zytogenetischen Untersuchung klonale chromosomale Aberrationen (Toyama et al. 1993, Solé et al. 2005, Haase et al. 2007, Haase 2008). Mit Erfassung des Karyotyps nicht nur zum Zeitpunkt der Erstdiagnose, sondern auch im Erkrankungsverlauf durch sequentielle 
zytogenetische Untersuchungen kann ein Progress der Erkrankung mit Einfluss auf die weitere Prognose festgestellt werden.

Die klinische Indikation zu zytogenetischen Verlaufsuntersuchungen ist bei Patienten gegeben, bei denen ein kuratives Therapiekonzept verfolgt wird, da durch eine zytogenetische Verlaufsuntersuchung ein Progress frühzeitig erkannt werden kann und ohne Zeitverlust zum Beispiel eine Knochenmarkspendersuche oder eine andere Therapieeskalation vorgenommen werden kann. Bei einem palliativen Ansatz ist die Untersuchung unter laufender Therapie sinnvoll, um einen eventuell auftretenden Wirkverlust der Therapie zu erfassen, um nicht länger als nötig mit einer möglicherweise unwirksamen und teuren Substanz zu therapieren. Nur so kann frühzeitig eine dem Krankheitsverlauf angepasste Therapie möglich gemacht werden

In den bisher veröffentlichen Prognose Scoring-Systemen sind die klonale Evolution und Evolutionsmuster nicht berücksichtigt. Mit dem international etablierten IPS-Score (Greenberg et al. 1997) kann beispielsweise das persönliche Risiko nicht eindeutig beurteilt werden. So wurden alle Patienten mit einer Aberration in die zytogenetische Subgruppe "intermediär" eingeteilt, wenn diese nicht den Chromosomenveränderungen der Einteilung „niedrig“ oder "hoch“ entsprachen. Im Rahmen einer Revision der zytogenetischen Risikogruppen (Schanz et al. 2012) auf der Basis eines wesentlich größeren Datensatzes wurden, statt der bisher drei, fünf zytogenetische Risikogruppen unterschieden. Dadurch wurde die prognostische Präzision erhöht und damit eine bessere Einschätzung des individuellen Risikos ermöglicht. In der vorliegenden Arbeit konnte gezeigt werden, dass die Karyotypevolution in Abhängigkeit von der klonalen Zusammensetzung und des Auftretens von neuen Anomalien die Prognose des Patienten deutlich verschlechtert. Da den unterschiedlichen Erkrankungsverläufen bestimmte zytogenetische Muster zugrunde liegen, sollten diese in Zukunft ebenfalls in Scoring-Systemen erfasst werden. 


\section{Zusammenfassung}

Myelodysplastische Syndrome (MDS) sind klonale Erkrankungen der hämatopoetischen Stammzellen, die sich im Krankheitsbild und Progressionsverhalten sehr heterogen darstellen. Der Progress der Erkrankung vollzieht sich im Rahmen einer Mehrschrittpathogenese. Die genetische Instabilität begünstigt die graduelle oder plötzlich auftretende Akkumulation von Aberrationen und damit die Entstehung einer klonalen Evolution. Diese spiegelt somit den Erkrankungsprogress wider (Haase 2005).

In der vorliegenden Arbeit wurde die prognostische Relevanz der klonalen Evolution sowie der unterschiedlichen Evolutionsmuster in Hinblick auf die unterschiedlichen klinischen Erkrankungsverläufe bei Patienten mit MDS und MDS-AL untersucht.

Dabei wurden retrospektiv die Daten von 729 Patienten ausgewertet. 225 Verlaufsuntersuchungen mit insgesamt 1208 Chromosomenanalysen wurden in der Auswertung berücksichtigt. Es konnte gezeigt werden, dass das Vorkommen einer klonalen Evolution mit einer ungünstigen Prognose assoziiert ist. So wiesen Patienten mit einer klonalen Evolution signifikant kürzere Überlebenszeiten als Patienten ohne klonale Evolution auf $(24,4$ Monate vs. 103,6 Monate, $p<0,01)$ und zeigten häufiger einen Übergang in eine $A M L$ (48,0\% vs. 21,4\%, $p<0,01)$. Der Übergang in eine AML geschah zudem in einem deutlich kürzeren Zeitintervall als bei Patienten ohne klonale Evolution (17,5 Monate vs. n.e., $p<0,01)$. Weiterhin konnte die klonale Evolution hinsichtlich des Zeitpunkts ihres Auftretens in eine frühe und eine späte klonale Evolution unterteilt werden (im Median nach 5,3 Monaten vs. 21,9 Monaten, $\mathrm{p}<0,01$ ). Dabei stellte sich heraus, dass auch der Zeitpunkt des Auftretens eine prognostische Bedeutung hat. Auch der Diagnosezeitpunkt einer klonalen Evolution stellt einen Prognosefaktor dar. Bei Nachweis einer klonalen Evolution bereits bei Erstdiagnose war die Prognose deutlich schlechter (8,2 Monate vs. 32,6 Monate, $\mathrm{p}<0,01)$. Innerhalb der Gruppe der Patienten mit klonaler Evolution bleibt der Einfluss des Verteilungstyps hinsichtlich der Prognose bestehen und erlaubt die Einteilung weiterer klinischer Subgruppen. Dabei waren die Zunahme der Klongröße (6,6 Monate vs. 18,0 Monate, $p=0,046)$ und das Auftreten von zusätzlichen Aberrationen im Verlauf (6,6 Monate vs. 19,5 Monate, $p=0,022)$ mit einer ungünstigen Prognose assoziiert. Die bisher beschriebenen Evolutionsmuster von Tricot et al. (1985), im Wesentlichen basierend auf den Blastenanteil, wurden unter Einbeziehung der Zytogenetik erweitert und insgesamt in fünf Hauptgruppen unterteilt. Anhand der erhobenen Primär- und Verlaufsdaten war es möglich, unterschiedliche prognostisch relevante klinische Verläufe zu erfassen und die be- 
schriebenen Evolutionsmuster von Tricot et al. (1985) bestimmten Chromosomenanomalien zuzuordnen. Als prognostisch ungünstig erwiesen sich die Index-Aberrationen Monosomie 7/7q-Deletion (8,2 Monate vs. 18,2 Monate, $p=0,020)$ und 17p- Anomalien (1,8 Monate vs. 17,9 Monate, $p=0,002)$ innerhalb der klonalen Evolution. Häufige Anomalien bei Nachweis einer klonalen Evolution waren die 5q-Deletion und 17p-Anomalien.

In den bisher veröffentlichten Prognose Scoring-Systemen sind die klonale Evolution und Evolutionsmuster nicht berücksichtigt. Ein Progress der Erkrankung kann durch sequentielle zytogenetische Untersuchungen festgestellt werden und somit frühzeitig eine dem Krankheitsverlauf angepasste Therapie möglich gemacht werden. Wie in dieser Arbeit gezeigt wurde, stellt die klonale Evolution und Evolutionsmuster einen wichtigen Prognosefaktor dar. Diese sollten in Zukunft ebenfalls in Scoring-Systemen erfasst werden, um damit eine bessere Einschätzung des individuellen Risikos zu ermöglichen. 


\section{Literaturverzeichnis}

Aul C, Gattermann N, Schneider W (1992): Age-related incidence and other

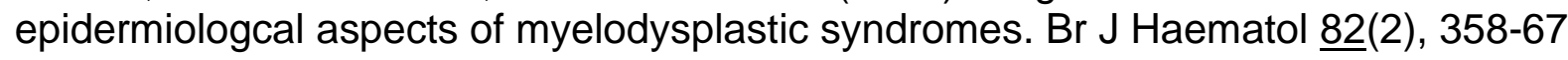

Aul C, Bowen DT, Yoshida Y (1998): Pathogenesis, etiology and epidemiology of myelodysplastic syndromes. Haematologica $\underline{83}(1), 71-86$

Benítez J, Carbonell F, Sanchez FJ, Heimpel H (1985): Karyotypic evolution in patients with myelodysplastic syndromes. Cancer Genet Cytogenet 16(2), 157-67

Benítez J, Martinez FC, Toledo C, Sanchez FJ, Ramos C (1987): Leukemic transformation in patients with the $5 q$ - alteration: analysis of the behaviour of the $5 q$ - clones in preleukemic to leukemic phases. Cancer Genet Cytogenet 26 (2), 199-207

Bennett JM (2000): World Health Organization classification of the acute leukemias and myelodysplastic syndrome. Int J Hematol $\underline{72}(2), 131-33$

Bennett JM, Catovsky D, Daniel MT, Flandrin G, Galton DA, Gralnick HR, Sultan C (1982):

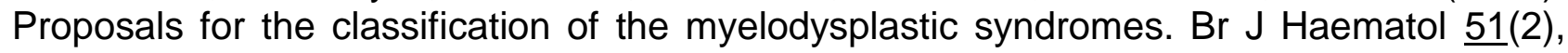
189-99

Bernasconi P, Klersy C, Boni M, Cavigliano PM, Giardini I, Rocca B, Zappatore R, Dambruoso I, Calvello C, Caresana $\mathrm{M}$ et al. (2010): Does cytogenetic evolution have any prognostic relevance in myelodysplastic syndromes? A study on 153 patients from a single institution. Ann Hematol $\underline{89}(6), 545-51$

Bloomfield CD, Goldman A, Hassfeld D, de la Chapelle A (1984): Fourth International Workshop on Chromosomes in Leukemia 1982: Clinical significance of chromosomal abnormalities in acute nonlymphoblastic leukemia. Cancer Genet Cytogenet 11(3), 332-50

Bochtler T, Stölzel F, Heilig CE, Kunz C, Mohr B, Jauch A, Janssen JW, Kramer M, Benner A, Bornhäuser M et al. (2013): Clonal heterogeneity as detected by metaphase karyotyping is an indicator of poor prognosis in acute myeloid leukemia. J Clin Oncol 31(31), 3898-905

Boultwood J, Lewis S, Wainscoat JS (1994): The 5q-syndrome. Blood 84(10), 3253-60

Boultwood J, Fidler C, Strickson AJ, Watkins F, Gama S, Kearney L, Tosi S, Kasprzyk A, Cheng JF, Jaju RJ et al. (2002): Narrowing and genomic annotation of the commonly deleted region of the 5q-syndrome. Blood 99(12), 4638-41

Braulke F, Schanz J, Götze K, Müller-Thomas C, Platzbecker U, Germing U, Brümmendorf T.H, Bug G, Giagounidis A, Nolte F et al. (2013): Karyotype evolution in MDS patients detected by CD34+ PB FISH- first results of the ongoing prospective multicenter German diagnostic study. Jahrestagung der Deutschen, Österreichischen und Schweizerischen Gesellschaft für Hämatologie und Onkologie, Wien. Onkologie $\underline{36}$ (suppl 7), 230

Brunning RD, Orazi A, Germing U, Le Beau MM, Porwit A, Baumann I, Vardiman JW, Hellstrom-Lindberg E: Myelodysplastic syndromes/neoplasms. In: Swerdlow SH, Campo E, Harris NL u.a (eds.): WHO Classification of Tumours of Haematopoietic and Lymphoid Tissues. IARC Press, Lyon 2008, 88-103 
Cermák J, Belicková M, Krejcová H, Michalová K, Zilovcová S, Zemanová Z, Brezinová J, Sieglová Z (2005): The presence of clonal cell subpopulations in peripheral blood and bone marrow of patients with refractory cytopenia with multilineage dysplasia but not in patients with refractory anemia may reflect a multistep pathogenesis of myelodysplasia. Leukemina Res 29 (4), 371-79

Cox DR (1972): Regression models and Life-Tables. JR Stat Soc 34(2), 187-220

Farrow A, Jacobs A, West RR (1989): Myelodysplasia, chemical exposure, and other environmental factors. Leukemia $\underline{3}(1), 33-35$

Gattermann N, Aul C, Schneider W (1990): Two types of acquired idiopathic sideroblastic anaemia (AISA). Br J Haematol $\underline{74}(1), 45-52$

Geddes AA, Bowen DT, Jacobs A (1990): Clonal karyotype abnormalities and clinical progress in the myelodysplastic syndrome. Br J Haematol $\underline{76}(12), 194-202$

Germing U, Haas R: 25 Jahre MDS-Register. In: Germing U und Haas R (eds.): Myelodysplastische Syndrome, Bilanz des aktuellen Wissens. Düsseldorf University Press, Düsseldorf 2009, 1-2

Germing U, Strupp C, Kündgen A, Bowen D, Aul C, Haas R, Gattermann N (2004): No increase in age-specific incidence of myelodysplastic syndromes. Haematologica $\underline{89}(8)$, 905-10

Germing U, Hildebrandt B, Pfeilstöcker M, Nösslinger T, Valent P, Fonatsch C, Lübbert M, Haase D, Steidl C, Krieger O et al. (2005): Refinement of the international prognostic scoring system (IPSS) by including LDH as an additional prognostic variable to improve risk assessment in patients with primary myelodysplastic syndromes (MDS). Leukemia 19 (12), 2223-31

Germing U, Kobbe G, Haas R, Gattermann N (2013): Myelodysplastic syndromes: diagnosis, prognosis and treatment. Dtsch Arztebl Int 110(46), 783-90, DOI: 10.3238/ arztebl.2013.0783

Giagounidis A: Myelodysplastische Syndrome. In: Mösser J (ed.): Der Internist. Springer Verlag, Berlin Heidelberg 2013, 54(6), 699-708, DOI: 10.1007/ s00108-012-3214-3

Giagounidis A, Germing U, Aul C (2006): Biological and prognostic significance of chromosome $5 q$ deletions in myeloid malignancies. Clin Cancer Res $\underline{12(1), 5-10}$

Giagounidis A, Germing U, Haase S, Hildebrandt B, Schlegelberger B, Schoch C, Wilkens L, Heinsch M, Willems H, Aivado M et al. (2004): Clinical, morphological, cytogenetic, and prognostic features of patients with myelodysplastic syndromes and $\operatorname{del}(5 q)$ including band q31. Leukemia 18(1), 113-19

González Manso AI, García Marcilla A, Barreiro E, Gilsanz F (1992): Cytohematologic and cytogenetic prognostic factors at diagnosis and in the evolution in 46 primary myelodysplastic syndromes. Cancer Genet Cytogenet $\underline{61}(2), 174-82$

Greenberg P, Cox C, Le Beau MM, Fenaux P, Morel P, Sanz G, Sanz M, Vallespi T, Hamblin T, Oscier D et al. (1997): International scoring system for evaluating prognosis in myelodysplastic syndromes. Blood $\underline{89}(6), 2079-88$ 
Greenberg P, Tuechler H, Schanz J, Sanz G, Garcia-Manero G, Solé F, Bennett JM, Bowen D, Fenaux P, Dreyfus $F$ et al. (2012): Revised international prognostic scoring system (IPSS-R) for myelodysplastic syndromes. Blood 120(12), 2454-65

Haase D: Untersuchungen zur Biologie von myelodysplatischen Syndromen und sekundären akuten myeloischen Leukämien, Bedeutung für Pathogenese und Erkrankungsverlauf. 1. Auflage; Cuvillier Verlag, Göttingen 2005. Zugl. Med. Habil. Schr. Göttingen 2002

Haase D (2008): Cytogenetic features in myelodysplastic syndromes. Ann Hematol $\underline{87}(7)$, 515-26

Haase D, Fonatsch C, Freund M (1992): Karyotype instability in myelodysplastic syndromes-a specific step in pathogenesis preceding clonal chromosome anomalies. Leuk Lymphoma $\underline{8}(3), 221-28$

Haase D, Feuring-Buske M, Schäfer C, Schoch C, Troff C, Gahn B, Hiddemann W, Wörmann B (1997): Cytogenetic analysis of CD34+ subpopulations in AML and MDS characterized by the expression of CD38 and CD117. Leukemia 11(5), 674-79

Haase D, Germing U, Schanz J, Pfeilstöcker M, Nösslinger T, Hildebrandt B, Kundgen A, Lübbert M, Kunzmann R, Giagounidis A et al. (2007): New insights into the prognostic impact of the karyotype in MDS and correlation with subtypes: evidence from a core dataset of 2124 patients. Blood 110(13), 4385-95

Haase D, Ganster C, Steidl C, Shirneshan K, Braulke F, Schanz J: Cytogenetics of MDS. In: Várkonyi J (ed.): The Myelodysplastic Syndroms. Springer Verlag, Dordrecht 2011, 5585

Haferlach C, Zenger M, Alpermann T, Schnittger S, Kern W, Haferlach T (2011): Cytogenetic Clonal Evolution in MDS is Associated with Shifts towards Unfavorable Karyotypes According to IPSS and Shorter Overall Survival: A Study on 988 MDS Patients Studied Sequentially by Chromosome Banding Analysis. Blood (ASH Annual Meeting Abstracts) 118: Abstract 968

Heim S, Mitelman F (1986): Chromosome abnormalities in the myelodysplastic syndromes. Clin Haematol 15(4), 1003-21

Hofmann WK, Platzbecker U, Götze K, Stauder R, Passweg J, Germing U (2013): Leitlinie Myelodysplastische Syndrome - Onkopedia. Deutsche Gesellschaft für Hämatologie und Onkologie: www.dgho-onkopedia.de/de/onkopedia/leitlinien/mds. Abgerufen am 11.03.2015

Horiike S, Taniwaki M, Misawa S, Abe T (1988): Chromosome abnormalities and karyotypic evolution in 83 patients with myelodysplastic syndrome and predictive value for prognosis. Cancer $\underline{62}(6), 1129-38$

Jabbour E, Takahashi K, Wang X, Cornelison AM, Abruzzo L, Kadia T, Borthakur G, Estrov Z, O'Brien S, Mallo M et al. (2013): Acquisition of cytogenetic abnormalities in patients with IPSS defined lower-risk myelodysplastic syndrome is associated with poor prognosis and transformation to acute myelogenous leukemia. Am J Hematol $\underline{88}(10), 831$ 37 
Jaffe ES, Harris NL, Stein H, Vardiman J (eds.): WHO Classification of Tumours: Pathology and Genetics of Tumours of Haematopoietic and Lymphoid Tissues. IARC Press, Lyon 2001

Janka-Schaub G, Haase D: Transfusionbedingte Eisenüberladung: Ursachen und Therapieprinzipien, In: Janka-Schaub G und Haase D (eds.): Therapie der Eisenintoxikation. Thieme Verlag, Deutschland 2009, 1-7

Janssen JW, Buschle M, Layton M, Drexler HG, Lyons J, van den Berghe $H$, Heimpel $H$, Kubanek B, Kleihauer E, Mufti GJ et al. (1989): Clonal analysis of myelodysplastic syndromes: evidence of multipotent stem cell origin. Blood $\underline{73}(1), 248-54$

Johansson B, Mertens F, Mitelman F (1996): Primary vs. secondary neoplasia-associated chromosomal abnormalities-balanced rearrangements vs. genomic imbalances? Genes Chromosomes Cancer 16(3), 155-63

Kaplan EL, Meier P (1958): Non-parametric estimation from incomplete observations. J Am Stat Assoc 푸, 457-81

Kuendgen A, Strupp C, Aivado M, Hildebrandt B, Haas R, Gattermann N, Germing U (2006): Myelodysplastic syndromes in patients younger than age 50. J Clin Oncol 24(34), 5358-65

Lai JL, Preudhomme C, Zandecki M, Flactif M, Vanrumbeke M, Lepelley P, Wattel E, Fenaux $P$ (1995): Myelodysplastic syndromes and acute myeloid leukemia with 17p deletion. An entity characterized by specific dysgranulopoïesis and a high incidence of P53 mutations. Leukemia $\underline{9}(3), 370-81$

Lai YY, Qiu JY, Zhang Y, Zhang Y, Shi Y, He Q, Dang H, Lu DP (2004): Clinical significance of continuous karyotyping in myelodysplastic syndromes. Zhonghua Xue Ye Xue $\mathrm{Za}$ Zhi 25 $(11), 645-48$

List A, Dewald G, Bennett J, Giagounidis A, Raza A, Feldman E, Powell B, Greenberg P, Thomas D, Stone R et al. (2006): Myelodysplastic Syndrome-003 Study Investigators. Lenalidomide in the myelodysplastic syndrome with chromosome $5 q$ deletion. $N$ Engl J Med 355(14), 1456-65

Malcovati L, Germing U, Kuendgen A, Della Porta MG, Pascutto C, Invernizzi R, Giagounidis A, Hildebrandt B, Bernasconi P, Knipp S et al. (2007): Time-dependent prognostic scoring system for predicting survival and leukemic evolution in myelodysplastic syndromes.J Clin Oncol 25(23), 3503-10

Malcovati L, Della Porta MG, Strupp C, Ambaglio I, Kuendgen A, Nachtkamp K, Travaglino $E$, Invernizzi R, Pascutto C, Lazzarino M et al. (2011): Impact of the degree of anemia on the outcome of patients with myelodysplastic syndrome and its integration into the WHO classification-based Prognostic Scoring System (WPSS Haematologica 96(10), 1433-40

Malcovati L, Hellström-Lindberg E, Bowen D, Adès L, Cermak J, Del Cañizo C, Della Porta MG, Fenaux P, Gattermann N, Germing U et al. (2013): Diagnosis and treatment of primary myelodysplastic syndromes in adults: recommendations from the European LeukemiaNet. Blood 122(17), 2943-64

Mallo M, Cervera J, Schanz J, Such E, García-Manero G, Luño E, Steidl C, Espinet B, Vallespí T, Germing $U$ et al. (2011): Impact of adjunct cytogenetic abnormalities for prog- 
nostic stratification in patients with myelodysplastic syndrome and deletion $5 \mathrm{q}$. Leukemia $\underline{25}(1), 110-20$

Marisavljevic D, Pantic M, Djordjevic V (2013): Only particular cytogenetic events are related to disease progression in sequential cytogenetic studies in myelodysplastic syndromes. J BUON $\underline{18}(2), 535-36$

Martínez-Ramírez A, Urioste M, Alvarez S, Vizmanos JL, Calasanz MJ, Cigudosa JC, Benítez J (2004): Cytogenetic profile of myelodysplastic syndromes with complex karyotypes: an analysis using spectral karyotyping. Cancer Genet Cytogenet 153(1), 39-47

Michaux JL, Martiat P (1993): Chronic myelomonocytic leukaemia (CMML)-a myelodysplastic or myeloproliferative syndrome? Leuk Lymphoma $\underline{9}(1-2), 35-41$

Nagarajan L (1995): Molecular analysis of the 5q- chromosome. Leuk Lymphoma 17(5-6), 361-66

Neukirchen J, Haas R, Germing U: Epidemiologie Myelodysplastischer Syndrome. In: Germing U und Haas R (eds.): Myelodysplastische Syndrome, Bilanz des aktuellen Wissens, Düsseldorf University Press, Düsseldorf 2009, 3-12

Neukirchen J, Schoonen WM, Strupp C, Gattermann N, Aul C, Haas R, Germing U (2011): Incidence and prevalence of myelodysplastic syndromes: data from the Düsseldorf MDSregistry. Leuk Res $\underline{35}(12), 1591-96$

Neuwirtová R, Mociková K, Musilová J, Jelínek J, Havlícek F, Michalová K, Adamkov M (1996): Mixed myelodysplastic and myeloproliferative syndromes. Leuk Res 20 (9), 717-26

Nisse C, Lorthois C, Drop V, Eloy E, Haguenoer JM, Fenaux P (1995): Exposure to occupational and environmental factors in myelodysplastic syndromes. Preliminary results of a case-control study. Leukemia $\underline{9}(4), 693-99$

Nolte F, Hofman WK: Myelodysplastische Syndrome: Molekulare Pathogenese und genetische Veränderungen. In: Germing U und Haas R (eds.): Myelodysplastische Syndrome, Bilanz des aktuellen Wissens, Düsseldorf University Press, Düsseldorf 2009, 71-84

Ohyashiki K, Iwabuchi A, Junko H, Ohyashiki T, Keisuke T: Cytogenetics of myelodysplastic syndromes with disease evolution and their clinical implications. In: Nomura T, Yoshida Y (eds.): Myelodysplastic Syndromes, Advances in research and treatment. Elsevier Verlag, Amsterdam 1995, 195-99

Olney HJ, Le Beau MM: The Cytogenetics and Molecular Biology of the Myelodysplastic Syndromes. In: Bennett JM (ed.): Myelodysplastic Syndromes: Pathobiology and Clinical Management. Marcel Dekker Inc., New York Basel 2002, 89-119

Parker JE, Mufti GJ (2001): The role of apoptosis in the pathogenesis of the myelodysplastic syndromes. Int J Hematol $\underline{73}(4), 416-28$

Pedersen B (1998): 5q(-) survival: importance of gender and deleted $5 q$ bands and survival analysis based on 324 published cases. Leuk Lymphoma 31(3-4), 325-34

Pellagatti A, Cazzola M, Giagounidis A, Malcovati L, Porta MG, Killick S, Campbell LJ, Wang L, Langford CF, Fidler C et al. (2006): Gene expression profiles of CD34+ cells in 
myelodysplastic syndromes: involvement of interferon-stimulated genes and correlation to FAB subtype and karyotype. Blood 108(1), 337-45

Peto R, Pike MC, Armitage P, Breslow NE, Cox DR, Howard SV, Mantle N, McPherson U, Peto J, Smith PG (1977): Design and analysis of randomized trials requiering prolonged observation of each patient. II. analysis and example. Br J Cancer $\underline{35}$ (1), 1-39

Platzbecker U, Schetelig J, Finke J, Trenschel R, Scott BL, Kobbe G, Schaefer-Eckart K, Bornhäuser M, Itzykson R, Germing $U$ et al. (2012): Allogeneic hematopoietic cell transplantation in patients age 60-70 years with de novo high-risk myelodysplastic syndrome or secondary acute myelogenous leukemia: comparison with patients lacking donors who received azacitidine. Biol Blood Marrow Transplant 18(9), 1415-21

Raskind WH, Tirumali N, Jacobson R, Singer J, Fialkow PJ (1984): Evidence for a multistep pathogenesis of a myelodysplastic syndrome. Blood $\underline{63}(6), 1318-23$

Raza A, Alvi S, Borok RZ, Span L, Parcharidou A, Alston D, Rifkin S, Robin E, Shah R, Gregory SA (1997): Excessive proliferation matched by excessive apoptosis in myelodysplastic syndromes: the cause-effect relationship. Leuk Lymphoma $27(1-2), 111$ 18

Sanz GF, Sanz MA, Vallespí T, Cañizo MC, Torrabadella M, García S, Irriguible D, San Miguel JF (1989): Two regression models and a scoring system for predicting survival and planning treatment in myelodysplastic syndromes: a multivariate analysis of prognostic factors in 370 patients. Blood $\underline{74}(1)$, 395-408

Schanz J: Charakterisierung und prognostische Bedeutung zytogenetischer Befunde bei myelodysplastischen Syndromen und sekundärer akuter Leukämie nach Myelodysplasie. Med. Diss. Göttingen 2003

Schanz J, Steidl C, Fonatsch C, Pfeilstöcker M, Nösslinger T, Tuechler H, Valent P, Hildebrandt B, Giagounidis A, Aul C et al. (2011): Coalesced multicentric analysis of 2,351 patients with myelodysplastic syndromes indicates an underestimation of poor-risk cytogenetics of myelodysplastic syndromes in the international prognostic scoring system. J Clin Oncol 29(15), 1963-70

Schanz J, Tüchler H, Solé F, Mallo M, Luño E, Cervera J, Granada I, Hildebrandt B, Slovak ML, Ohyashiki K et al. (2012): New comprehensive cytogenetic scoring system for primary myelodysplastic syndromes (MDS) and oligoblastic acute myeloid leukemia after MDS derived from an international database merge. J Clin Oncol 30(8), 820-29

Sebaa A, Ades L, Baran-Marzack F, Mozziconacci MJ, Penther D, Dobbelstein S, Stamatoullas A, Récher C, Prebet T, Moulessehoul S et al. (2012): Incidence of 17p deletions and TP53 mutation in myelodysplastic syndrome and acute myeloid leukemia with $5 q$ deletion. Genes Chromosomes Cancer $\underline{51}(12), 1086-92$

Shaffer LG, McGowan-Jordan J, Schmid M: An International System for Human Cytogenetic Nomenclature. Recommendations of the International Standing Committee on Human Cytogenetic Nomenclature. Karger, Basel, 2013

Solé F, Espinet B, Sanz GF, Cervera J, Calasanz MJ, Luño E, Prieto F, Granada I, Hernández JM, Cigudosa JC et al. (2000): Incidence, characterization and prognostic significance of chromosomal abnormalities in 640 patients with primary myelodysplastic syndromes. Br J Haematol 108(2), 346-56 


\section{Literaturverzeichnis}

Solé F, Luño E, Sanzo C, Espinet B, Sanz GF, Cervera J, Calasanz MJ, Cigudosa JC, Millà F, Ribera JM et al. (2005): Identification of novel cytogenetic markers with prognostic significance in a series of 968 patients with primary myelodysplastic syndromes. Haematologica $\underline{90}(9), 1168-78$

Steidl C, Schabla R, Germing U, Hildebrandt B, Noesslinger T, Pfeilstoecker M, Giagounidis A, Kunzmann R, Haas P, Luebbert M et al. (2005): Sequential Cytogenetic Analyses of 577 Patients with Myelodysplastic Syndromes: Correlations between Initial Karyotype, Cytogenetic Dynamics, and Clinical Course. Blood (ASH Annual Meeting Abstracts) $\underline{106}$, Abstract 2531

Stephens PJ, Greenman CD, Fu B, Yang F, Bignell GR, Mudie LJ, Pleasance ED, Lau $\mathrm{KW}$, Beare D, Stebbings LA et al. (2011): Massive genomic rearrangement acquired in a single catastrophic event during cancer development. Cell 144(1), 27-40

Tien HF, Wang CH, Chuang SM, Lee FY, Liu MC, Chen YC, Shen MC, Lin KH, Lin DT (1995): Acute leukemic transformation of myelodysplastic syndrome- immunophenotypic, genotypic, and cytogenetic studies. Leuk Res $\underline{19}(9)$, 595-603.

Toyama K, Ohyashiki K, Yoshida Y, Abe T, Asano S, Hirai H, Hirashima K, Hotta T, Kuramoto A, Kuriya $S$ et al. (1993): Clinical implications of chromosomal abnormalities in 401 patients with myelodysplastic syndromes: a multicentric study in Japan. Leukemia $\underline{7}(4), 499-508$

Tricot G, Boogaerts MA, De Wolf-Peeters C, Van den Berghe H, Verwilghen RL (1985): The myelodysplastic syndromes: different evolution patterns based on sequential morphological and cytogenetic investigations. Br J Haematol $\underline{59}(4), 659-70$

Van den Berghe H, Michaux L (1997): 5q-, twenty-five years later: a synopsis. Cancer Genet Cytogenet $\underline{\text { 94}(1), ~ 1-7 ~}$

Volkert S, Kohlmann A, Schnittger S, Kern W, Haferlach T, Haferlach C (2014): Association of the type of $5 q$ loss with complex karyotype, clonal evolution, TP53 mutation status, and prognosis in acute myeloid leukemia and myelodysplastic syndrome. Genes Chromosomes Cancer $\underline{53}(5)$, 402-10

Vardiman JW, Bennett JM, Bain BJ, Baumann I, Thiele J, Orazi A: Myelodysplastic/ myeloproliferative neoplasm, unclassifiable. In: Swerdlow SH, Campo E, Harris NL u.a. (eds.): WHO classification of tumours of haematopoietic and lymphoid tissues. IARC press, Lyon 2008, 85-86

Wang H, Wang XQ, Xu XP, Lin GW (2010): Cytogenetic evolution correlates with poor prognosis in myelodysplastic syndrome. Cancer Genet Cytogenet 196(2), 159-66

White AD, Hoy TG, Jacobs A (1994): Extended cytogenetic follow-up and clinical progress in patients with myelodysplastic syndromes (MDS). Leuk Lymphoma 12(5-6), 401-12

Williamson PJ, Kruger AR, Reynolds PJ, Hamblin TJ, Oscier DG (1994): Establishing the incidence of myelodysplastic syndrome. Br J Haematol $\underline{87}(4), 743-45$

Zemanova Z, Michalova K, Buryova H, Brezinova J, Kostylkova K, Bystricka D, Novakova M, Sarova I, Izakova S, Lizcova L et al. (2014): Involvement of deleted chromosome 5 in complex chromosomal aberrations in newly diagnosed myelodysplastic syndromes (MDS) is correlated with extremely adverse prognosis. Leuk Res $\underline{38}(5), 537-44$ 
Publikation, die sich auf diese Arbeit bezieht

Cevik N, Haase D, Schanz J (2013): Prognostic relevance of cytogenetic evolution and the influence of the clonal composition in patients with myelodysplastic syndroms, Jahrestagung der Deutschen, Österreichischen und Schweizerischen Gesellschaft für Hämatologie und Onkologie, Wien. Onkologie $\underline{36}$ (suppl 7), 126 


\section{Anhang}

\subsection{Abbildungsverzeichnis}

Abbildung 1: Exemplarische Darstellung einer klonalen Evolution...................................

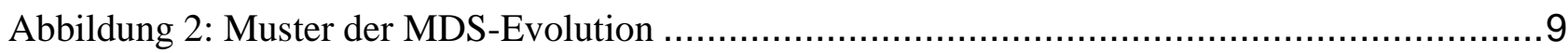

Abbildung 3: Häufigkeitsverteilung der MDS-Subgruppen gemäß der FAB-Klassifikation im

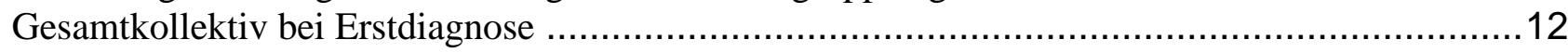

Abbildung 4: Klonale Evolution nach Geschlecht ............................................... 18

Abbildung 5: Häufigkeit klonaler Evolution abhängig von der Ätiologie .................................19

Abbildung 6: Kumulierte Überlebensanteile (Kaplan-Meier) der Gesamtgruppe ......................25

Abbildung 7: Kumulierte Überlebensanteile (Kaplan-Meier) in Abhängigkeit vom

Diagnosezeitpunkt der klonalen Evolution

Abbildung 8: Aberrationen pro Fall in Abhängigkeit vom Diagnosezeitpunkt der klonalen Evolution

Abbildung 9: Auftreten klonaler Evolution im zeitlichen Verlauf: Frühe und späte klonale Evolution

Abbildung 10: Kumulierte Überlebensanteile (Kaplan-Meier) in Abhängigkeit vom

Evolutionszeitpunkt: Frühe und späte klonale Evolution.

Abbildung 11: Häufigkeit komplexer Aberrationen in Abhängigkeit vom Auftreten klonaler Evolution.

Abbildung 12: Kumulierte Überlebensanteile (Kaplan-Meier) in Abhängigkeit vom Auftreten komplexer Aberrationen mit oder ohne zusätzliche klonale Evolution

Abbildung 13: Verteilungstyp in Abhängigkeit vom Auftreten klonaler Evolution

Abbildung 14: Klongröße in Abhängigkeit vom Auftreten klonaler Evolution

Abbildung 15: Kumulierte Überlebensanteile (Kaplan-Meier) in Abhängigkeit von der klonalen Zusammensetzung und dem Auftreten klonaler Evolution

Abbildung 16: Häufigkeit der AML in Abhängigkeit vom Auftreten klonaler Evolution

Abbildung 17: AML-freies Überleben der Gesamtgruppe

Abbildung 18: AML-freies Überleben in Abhängigkeit vom Evolutionszeitpunkt: Frühe und späte klonale Evolution

Abbildung 19: AML-freies Überleben in Abhängigkeit von der klonalen Zusammensetzung und dem Auftreten klonaler Evolution

Abbildung 20: Häufigkeit klonaler Aberrationen an einzelnen Chromosomen innerhalb der Gruppe von Patienten mit klonaler Evolution. 
Abbildung 21: Häufigkeit einer 5q-Deletion in Abhängigkeit vom Auftreten klonaler Evolution ..40

Abbildung 22: Kumulierte Überlebensanteile (Kaplan-Meier) für Patienten mit 5q-Deletion in Abhängigkeit vom Auftreten klonaler Evolution .

Abbildung 23: Kumulierte Überlebensanteile (Kaplan-Meier) für Patienten mit klonaler Evolution in Abhägigkeit vom Auftreten einer 5q-Deletion.

Abbildung 24: Häufigkeit einer totalen oder partiellen Monosomie 7 in Abhängigkeit vom Auftreten klonaler Evolution

Abbildung 25: Kumulierte Überlebensanteile (Kaplan-Meier) für Patienten mit klonaler Evolution in Abhägigkeit vom Auftreten einer totalen oder partiellen Monosomie 7 ....

Abbildung 26: Häufigkeit einer Trisomie 8 in Abhängigkeit vom Auftreten klonaler Evolution ....44

Abbildung 27: Kumulierte Überlebensanteile (Kaplan-Meier) für Patienten mit klonaler Evolution in Abhägigkeit vom Auftreten einer Trisomie 8

Abbildung 28: Häufigkeit einer 17p-Anomalie in Abhängigkeit vom Auftreten klonaler Evolution

Abbildung 29: Kumulierte Überlebensanteile (Kaplan-Meier) für Patienten mit klonaler Evolution in Abhägigkeit vom Auftreten einer 17p-Anomalie

Abbildung 30: Häufigkeit einer 20q-Deletion in Abhängigkeit vom Auftreten klonaler Evolution.47

Abbildung 31: Kumulierte Überlebensanteile (Kaplan-Meier) für Patienten mit klonaler Evolution in Abhägigkeit vom Auftreten einer 20q-Deletion....

Abbildung 32: Häufigkeit eines Y-Chromosomenverlustes in Abhängigkeit vom Auftreten klonaler Evolution .48

Abbildung 33: Kumulierte Überlebensanteile (Kaplan-Meier) für Patienten mit klonaler Evolution in Abhägigkeit vom Auftreten eines Y-Chromosomenverlustes.

Abbildung 34: Häufigkeit klonaler Evolutionsmuster ..... .50

Abbildung 35: Häufigkeit distinkter Aberrationen in Abhängigkeit der klonalen Evolutionsmuster

Abbildung 36: Kumulierte Überlebensanteile (Kaplan-Meier) klonaler Evolutionsmuster . 53

Abbildung 37: Muster der klonalen Evolution gemäß Tricot et al. 1985, modifiziert. .66

\subsection{Tabellenverzeichnis}

Tabelle 1: FAB-Klassifizierung der Myelodysplastischen Syndrome .2

Tabelle 2: WHO-Klassifikation Myelodysplastischer Syndrome 4

Tabelle 3: Revision des International Prognostic Scoring System (IPSS-R) .5 


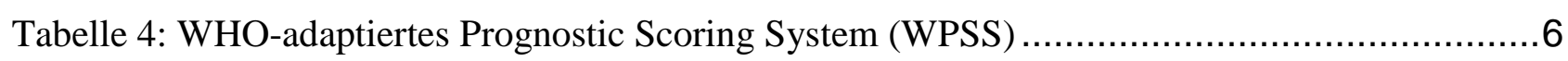

Tabelle 5: Häufigkeit klonaler Evolution in den Altersgruppen ...................................... 19

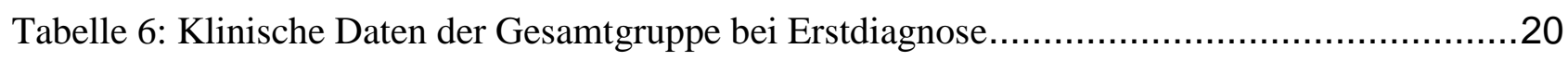

Tabelle 7: Klinische Daten der Patienten ohne klonale Evolution mit Verlaufsdaten ...................21

Tabelle 8: Klinische Daten der Patienten mit klonaler Evolution bei Erstdiagnose ....................23

Tabelle 9: Klinische Daten der Patienten mit klonaler Evolution im Krankheitsverlauf ..............24

Tabelle 10: Aberrationen pro Fall in Abhängigkeit von klonaler Evolution ...........................30

Tabelle 11: Häufigkeiten komplexer Aberrationen innerhalb klonaler Evolutionsmuster .............51

Tabelle 12: Die Zytogenetik der Patienten mit klonaler Evolution im Krankheitsverlauf .............54

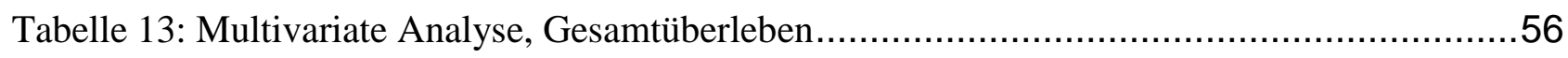

Tabelle 14: Multivariate Analyse, AML-freies Überleben .............................................5

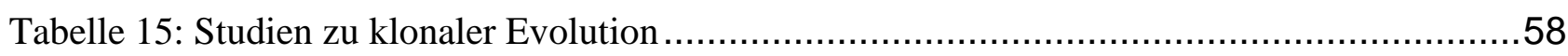

Tabelle 16: Primäre und sekundäre Aberrationen bei MDS gemäß Johansson et al. 1996, Mallo et

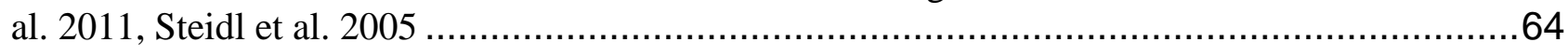

Tabelle 17: Primäre und sekundäre Aberrationen bei klonaler Evolution, eigene Daten...............65 


\section{Danksagung}

Danken möchte ich Herrn Prof. Dr. med. D. Haase, dem Leiter der Diagnostiklabore der Klinik für Hämatologie und Medizinische Onkologie, für die Vergabe des Themas und damit die Möglichkeit für mich, diese Studie durchführen zu können.

Mein ganz besonderer Dank gilt Frau PD Dr. med. J. Schanz für ihre Betreuung und die Unterstützung dieser Arbeit. Sie stand mir mit ihrer wertvollen Kritik und vielen Anregungen zur Seite und war am erfolgreichen Abschluss dieser Arbeit beteiligt.

Mein ganz besonderer Dank gilt auch Herrn Dr. med. R. Brans für die Durchsicht der vorliegenden Arbeit und für die Verbesserungsvorschläge.

Den Mitarbeitern/innen des Instituts für Medizinische Statistik, Universitätsmedizin Göttingen, danke ich sehr für die Beratungen bei den statistischen Analysen. 